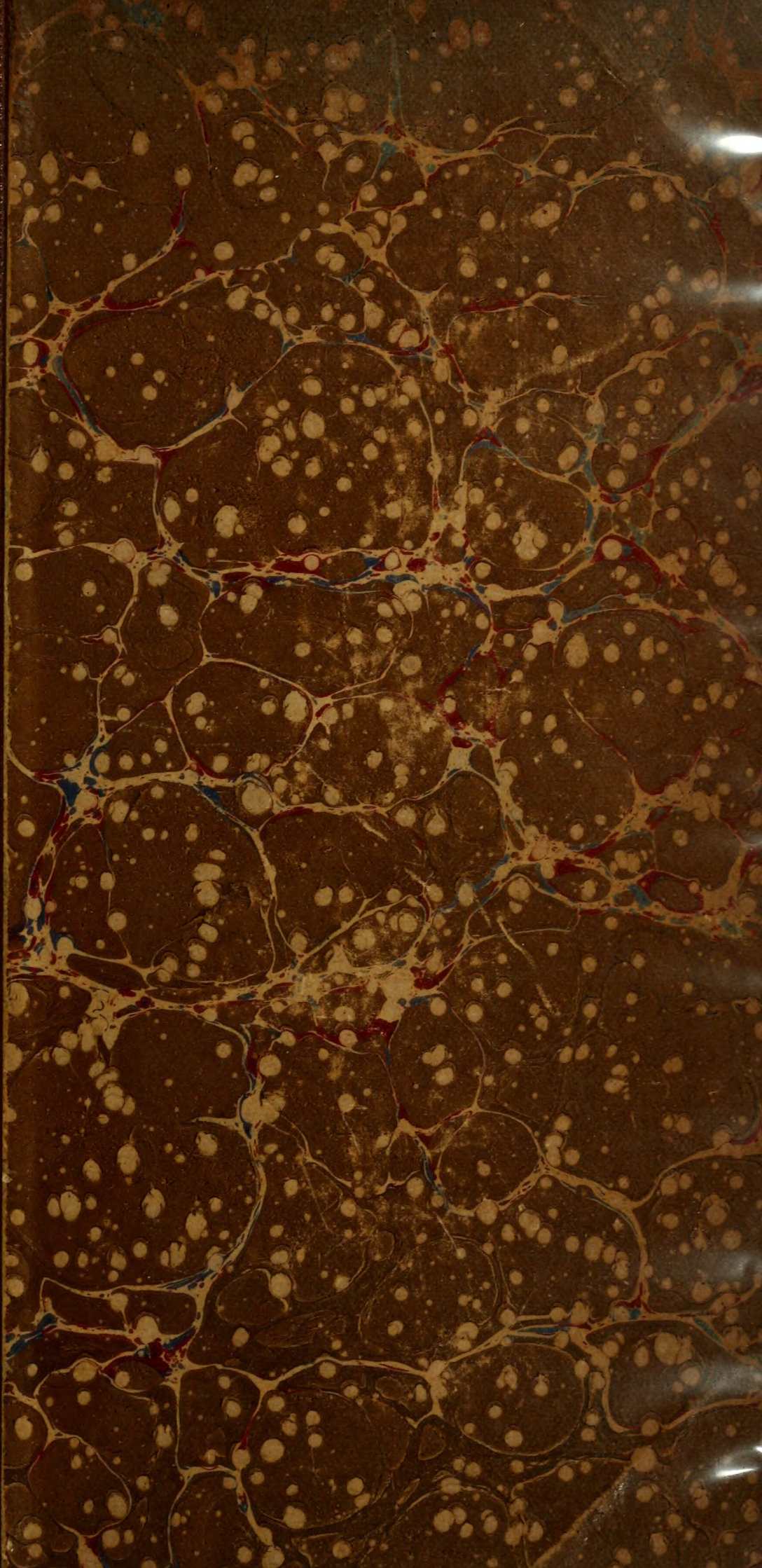




\section{toK110 \\ -E18}

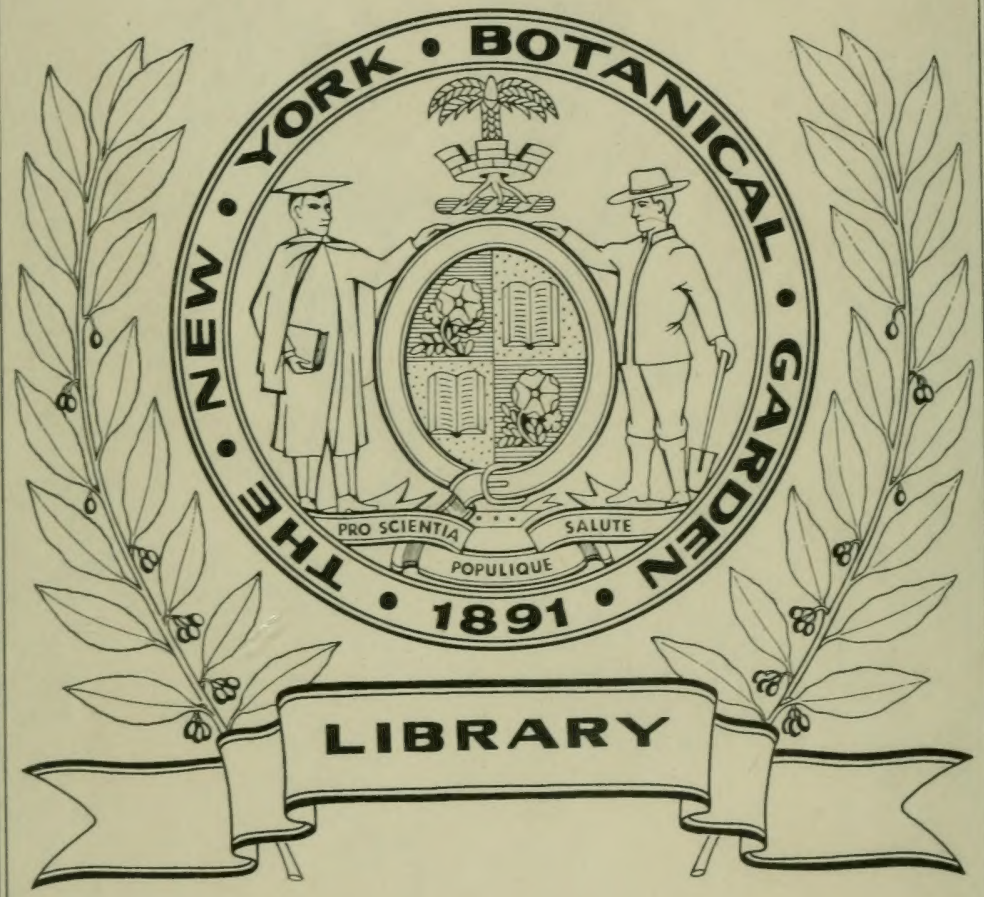




$$
\text { . }
$$ 



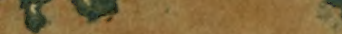




1

\section{MANUAL OF BOTANY}

5on

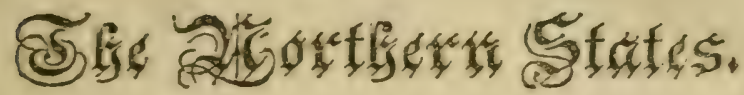

COMPRISIXG

CETERIC DESCRIPTIONS OF ALL PEEXOGAMOCS AKD CRTPTO-

GATOUS PLANTS TO THE NORTH OF VIRGIKIA, HITH-

ERTO DESCRIBED; WITH REFERENCES TO

THE NATURAL ORDERS OF IIY-

NEUS AND JUSSIEU.

Each Genus is further illustrated by short Descriptions of its most common Species.

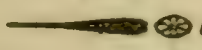

Br the Members of the Botantcal Clags ix WILLIAMS' COLLEGE, (Mass.)

From a Manuscript System, COMPILED BY TRE AUTHOR OF

RICHARD'S BOTANICAL DICTIOYARY.

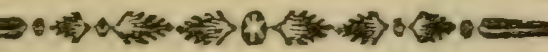

$$
\mathcal{L} B \mathscr{N} Y \text { : }
$$

RRITTED BI WEBSTERS AXD SEIXYERS. 


\title{
TO THE AUTHOR
}

\author{
or:
}

\section{RICHARD'S BOTANICAL DICTIONARY.}

\section{Dear Srr,}

WE consider ourselves happy in having an opportunity to express our gratitude for the assiluity and care, which you have manifested for our improvement in Natural Science. As the course of Lectures on Mineralegy, which you have conducted so entirely to the satisiaction of the Faculty of this College, and so much to the berefit of your class, is nearly completed, we are gratified with the prospect of attending your course of Lectures on Botany-innowing that our improvement in that branch of Natural Science would be greatly facilitated by a systematic description of regetables, and being destitute of such a system, we render you our thanks for the one, which you have been pleased, gratuitously, to present to us for publication.

Accept, dear Sir,

The assurances of our respect and esteem.

Trilliams' College, 8th April, 1817.

II. Walker Bishop,

Dor'us L. Clark,

IVilliam Eastman,

Luther Hainilton,

Lyman James,

Hedcul Pomeroy,

Emory C. Washbum,

Selah R. Jrms,

Daniel D. Banard,

Homer Bartlett,

Chandler Bates,
Charles Dillingham,

William $\mathcal{A}$. Hablock,

Gerard H. IIallock,

Anson T. Hooker,

Cyrus M. Lazell,

Lucas Morgan,

Joseph P. Mosher,

William Richards,

IIervey Smith,

Thomas Spring,

B. Horace Slarkweather, 
Lemuel P. Bates,

George $\boldsymbol{W}$. Benedict,

Daniel I. Betts,

David L. Coe,

Joshun N: Danforth,

Ebenezer Emmons,

Joseph H. Estabrook,

Charles Fitch,

Parker L. Hall,

Gardner Hayden,

Leicester Lloyd,

Thomas Peck,

William A. Porter,

Joln B. Skinncr,

Royal H. Smith,

William Wells,

Juhn Whilon,

John C. Brighan,

Nelson Brown,

Elijah H. Burvit,

Sammel Dickinuson,
Alvan Alvort,

Charles Baker,

Drwight Baldwin,

Johnson Baldirvin,

Andrerv Bumham,

Benjamin F. Clark,

Gardner Dorrance,

Jonathan Ely,

Simon C. Ezver's,

- Abner Forbes,

Mason Fussell,

William Gildersleeve,

Edivard Hooker,

William IV. Hunt,

Royal Joy,

William C. Kittrige,

John C. MLrgan,

Isaac Oakes,

Elijah Thayer,

Alvan Wheeler. 



\section{PREF.CE.}

EVERY one, who has passed through the first struggles of a student in pracicil botany, recollects the embarrassments in which he was perpetually involved, by the muititude of foreign genera contained in the European systems.Doctor Bigelow's Boston Flora proves the rery great relief which is afforded to the student by a system of plants limit. ed to his own particular clistrict. But there is a middle way in this as in other things. The northern section of the $\mathrm{Cni}$ ted States is sufficiently contracted for this object; and the common cultivated exotics ought certainly to be included.

Another obstacle to the progress of the student is the very concise generic characters given in most of the wolks of this nature. If he sets out witl the right class, is led to the right order, and fixes at last upon the right genus, his work is reduced to a manageable siate. To guicle him thrcugh those three steps is the object of this Marual. To effect this with ease to the learner, the common English names, the rnost striking specific characters, tosether with a few popular references, and the natural orders of Linneus and Jussieu, have been annexed to each generic description.

The generic characters of the phenogamous plants are translated from Persoon, and arranged principally upon his method. Additions and correcions were mide, howerer, on comparing the translation with the systems of Linneus, Pursh, Muhleibers, Willdenow, Ligrelow, the Hortus Kewen. sis, the Encycioprodia, and the Synopsis of Americrus Generar.

A. 2 


\section{vi}

The descriptions of cryptogamous plants are chiefly from Sprengel, and Doctor Smith's translation of Acharius. The orders, Algae and Fungi, are mostly from Turton and Lin. neus.

This work should be accompanied by the Botanical Dictionary, published at New-Havcn, which was translated from the French of Professor Richard; and it is so constructed that it may be bound in the same volume. 


\section{$(1)$.}

\section{LTNNEAN SYSTEM OF VEGETABLES.}

All Vegetables are divided into twenty-two* crasses. These classes are divided into orders. Ordens are divided into grafra. Gexera are diviled into specres. Specres are frequently changed into rarieties. Tarieties, however, are more properly within the province of the Gardener, than of the Botanist; at least the method of procuring varieties.

When a Botanist sees a plant, which he never saw before, and wishes to know its name and uses; he proceeds as follows.

1. He takes the unknown flower in his hand (no unknown plant can be ascertained without the flower) and compares its parts with the description of each class, until he finds the class to which it belongs.

2. He then goes to the orders of that class and finds its order in the same way.

3. Next he goes to the genera of that order, and reads their descriptions, until he finds the genus to which it belongs.

4. At last looks over the species of that genus, until he finds the exact description of his plant.

5. Thus he finds the Apple to be Crass 12, ORDER 5, GE. zus Pyrus, Specres Malus.

* Linneus divided them into 24 classes. But farther diso coveries, since his death, have proved the classes Polyadelphia and Polygamia to be too uncertain and rariable to be any longer retained. Persoon, therefore, and other eminent Botanists, hare rejected them. See these classes in the Dirtionary. 


\section{(2) \\ LINNEAN CLASSES.}

1. Morasimi, 1 stamen, or one sessile anther in the forter.

2. Draxphra, 2 stamens, or 2 sessile anthers.

3. Triaxdra, 3 stamens, or 3 sessile anthers.

4. Tetrannita, 4 stamens, or 4 sessile anthers.

5. Pextaxonis, 5 stamens, or 5 sessile antherg.

6. Hexamnis, 6 stamens, or 6 sessile anthers.

7. Heptaxdris, 7 stamens, or 7 sessile anthers,

8. Octaxdris, 8 stamens, or 8 sessile anthers.

9. ExyEarorid, 9 stamens, or 9 sessile anthers.

10. Decandria, 10 stamens, or 10 sessile anthers.

11. Dodecasturta, 12 to 19 stamens, or sessile anthers.

12. Icosandria, about 20 , or more, standing on the calyx.

13. Polyandria, always 20 or more, on the receptacle.

14. Didramia, 4 stamens, 2 of them uniformly the longest.

15. Tetranxama, 6 stamens, 4 of them uniformly the longest.

16. Moraderima, stamens united by their filaments in one set, anthers remaining separate.

17. Drapelpuia, stamens united by their filaments in two sets (sometimes in one set) flowers papilionaccous.

18. Srxigxests, stamens 5 , united by their anthers in one set, flowers compound.

19. Grandina, stamens stand on the germ, style, or stig. $\mathrm{ma}$, separate from the base of the calyx and corol.

20. Moxoecta, stamens and pistils in separate flowers, on the same plant.

21. Dioecra, stamens and pistils on separate plants.

22. Crxptogania, stamens and pistil so obscure that the plants can orily be classed by natural families. 


\section{ESPRAYATIONS FOR THE PRECRDING PAQE.}

Mon. Monogynia, 1 style,' or 1 sessile stigma.

Dig. Digynia, 2 styles, \&c.

Tri. Trigynia, 3 styles, \&c

Tet. Tetragynia, 4 styles, \&cc.

Pen. Pentagynia, 5 styles, \&c.

Hex. Hexagynia, 6 styles, \&c.

Hep. Heptagynia, 7 styles, \&c.

Dec. Decagynia, 10 styles, \&c.

Pol. Polygynia, more than 10 styles, \&x.

Gym. Gymnospermia, seeds naked.

Ang. Angiospermia, seeds in capsules.

Silic. Siliculosa, having pods whose length and breadih are nearly equal.

Siliq. Siliquosa, having pods whose lengths are more than double their breadths.

In the $16 \mathrm{th}, 17 \mathrm{th}, 19 \mathrm{th}, 20 \mathrm{hh}, 21$ st classes, the names and characters of preceding classes are taken for order's. In which, Mon. Monandria. Dia. Diandria. Tri. Triandiria. Tet. Tetrandria. Pen. Pentandria. Hex. Hexandria. Oct. Octandria. Dec. Decandria. Pol. Polyandria. Mon. Mon. adelphia.

In the 18th class. 1. Pol. Eq. Polygamia Equalis. 2. Pol. Sup. Polygamia Superfua. 3. Pol. Frus. Polygamia Frustranea. 4. Pol. Nec. Polygamia Necessaria. 5. Pol. Seg. Polygamia Segregata.

The $1 \mathrm{st}$ order in the 18th cliss is distinguished by haring all the forets perfect. 'The $2 \mathrm{~d}$, by having those of the disk perfect, while those of the ray are pistilkate. The $3 \mathrm{~d}$, by having those of the disk perfect, while those of the ray are neutral. The 4th, by having those of the clisk staminate, while those of the ray are pistillate. The 5 th, by having the florets all perfect, while each floret has a peritmith of its own.

In the $22 \mathrm{~d}$ class, the orders are distinguished by notural family characters. 1. Filices, (ferns) which bear fruit on the back of the leaves, or in which some part of the leaves scem as it were metamorphosed into a kind of fruit-berringspike. 'The appendix to this order includes the Pterinrles, which bear fruit on a peculiar appendage. 2. Mifusci, (mosses) which bear, on leafy stems and branches, one-celleci capsules, opening at the top, where they are covered by a peculjiar lid. 3. Hepaticae, (liverworts) witich beai, on herbaceous fronds, four-celled capsules opening with four vulves. 4. 
Algae, (seaweeds, \&c.) which bear, in an aquatic or gelatine ous frond, vesiculous or filamentous fruit. 5. Lichenes, (lichens) which bear, on fibrous, compact or gelatinous fronds, scutellae, patellulae, peltae, thalamia, disks or pilidia, containing the fructification. 6. Fungi, (mushroom, \&c.) which are destitute of herbage, consisting of a spungy, pulpy, leathery or woody substance, and bear fruit in a naked dilated membrane, or within the substance of the plant.

\section{Explatationg of the ciraracters axiexed to THE GEx- ERIC DESCRIPTIONS.}

The first number following the generic description is the number of the natural order of Linneus, to which the genus belongs; the second number is that of Jussieu. By referring to the natural orders of Limneus in the Botanical Dictionary, the qualities of plants may be so far ascrtained, as they depend on these natural affinities.

(9) annual. 5 biennial. 24 perennial. $h$ woody.

$p$, when the corol is purple. $r$, red. $w$, white. $y$, yellow. g, greenish. e, exotic.

* Placed before one or more generic nouns, at the end of 2n order, indicates that these genera sometimes vary from the classes and orders to which they belong, and are found to possess the characters of the class and order undex which their names are thus set down. 



\section{A \\ SYSTEM OF GENERA}

EOR THE

\section{NORTHERN STATES.}

\section{CLASS I. MONANDRIA.}

\section{Order I. Monogyina.}

Salicornia. Calyx infated, entire: corol o: seed 1 , inclosed in the calyx. 12.29-virginica, (samphire) branches undivided-herbacea, divided. .

Hippuris. Calyx superior, obsolete, with a 2. lobed margin : corol o : seed 1 : stigma simple. 15. 6.-Vulgaris, (marestail) leaves linear, whorled. $2 \%$.

* Scirpus, Cyperus.

Order II. Digtinta.

Calittriche. Calyx inferior, 2.leaved : caipo sule membranaceous and margined: (flowers sometimes monoecoius, and by some the caly $\mathrm{x}$ is called the corol.) 12. 6-autumnalis, (water-starwort) leaves all linear-intermedia, stem-leaves linear only-verna, flowers androgynous.

Burrum. Calyx 3-cleft, berry-like: corol o: seed 1. 12. 29.-cafitatum, (blite) heads alternate -virgatum, heads scattered. (-) r.

CInxA. Calyx a 1 -flowered glume, 2-valved: corol a 2-valved glume: seed 1. 4. 10-arundinacea, panicle large, many-flowered. 24.

* Uniola. 


\section{CLASS II. DIANDRIA:}

\section{ORDER I. Monogynis.}

A. Corol inferior, 1-petalled regular: border, exceft in Jasminum, 4-parted.

JAsmivuar. Corol salver-form, 5 to 8 -cleft: berry 2 -seeded, each seed solitary, arilled. 44.37. -officinale, (jasmine) leaves pinnate. $h$.w. e.

Ligustrum. Calyx 4-toothed: corol with ovate divisions: berry 4-seeded. 44.37-vulgare (prim) panicled. h. w.

Syrirga. Corol salver-form : capsule 2 -celled. 44. 37-vulgaris, (lilac) leaves cordate. $h \cdot p$. and $w . e$.

B. Corol inferior, 1-hetalled, irregular : fruit cafsular.

UTricularia. Calyx 2-leaved, equal : coral ringent, spurred: capsule 1-celled, globular. 21. 34-vulsaris (bladder-wort) floats with air-blad. ders. $\psi$. $y$.

Catalpa. Corol 5-cleft: calyx 2-leaved : capsule 2-celled. 40.45-syringaefulia, (catalpa tree) leaves cordate. h. w. p. y.

Justicia. Calyx simple or clouble, 5.parted: corol ringent or nearly equal: capsule 2-celled, busting with an elastic claw: partitions transverse. 40. 35-adhatoda, (malabar nut) leaves lance-ovate. h. p.

Gratrolı. Calyx 7.leaved, the 2 outer ones spreading: corol 4-cleft, 2-lipped, reversed: stamens 4 ; ( 2 of them barren) stigma 2-lipped : capsule 2-celled. 40. 40-officinalis, (hedge-hyssop) leaves slightly toothed. [This is the aurea of Muhlenberg.] (O. $y$.

VERONICA. Calyx 4-parted: corol 4-cleft, lower division smaller: capsule 2 -celled. 40.35-serfyl- 


\section{CLASS II. ORDER IT.}

fijolia, (speedwell) racemes terminal. h. w. b. Scutellata, racemes lateral. 24 . w. b.

C. Corol inferior, 1-pzetalled, irregular: seeds naked.

Lycopus. Calyx tubular, half 5-cleft: corol tubular, 4.cleft, nearly equal, I division emarginate : stamens distant : seeds d, retuse. 42.39-e tu= ropeus, (water-horehound) leaves below gashtoothed, above serrate. 24-virginicus, leaves equally serrate, base narrow. $2 f . w$.

Monarda. Calyx cylindric striated, 5-toothed: corol ringent, upper lip linear, involving the filaments. 42. 39-didyma, (Oswego tea) heads in whorls. 4.r.-allofinylla, (mountain mint) leaves acute-serrati. $4 \cdot$.

Rosmarinus. Corol ringent, upper lip 2-parted : filaments long, curved, simpie, with a tooth. 42. 39-officinalis (rosemary) leaves hairy beneath.

Salvia. Calyx tubular, 2-lipped, underlip 2toothed: corol ringent: filaments transversely affixed to a pedicel. 42. 39-officinalis, (sage) leaves crenate. 24. b. p.e.-urticuefolia, leaves doublyserrate. $24 . \mathrm{b}$.

Collinsonia. Calyx tubular, 2-lipped : corol unequal, underlip many-cleft, capillary : one perfect seed. 40. 39-canadensis, (horse balm) leaves heart-ovate. $21 \cdot y$.

\section{Corol suherior.}

Circaea. Calyz 2-leaved: corol 2-petalled: capsule hispid, 2-celled, not gaping; cells 1-seeded. 48. 88-lutetiana, (enchanter's nightshade) leaves ovate, a little toothed. 2\%.w.

* Cunila, Schoenus, Scirpus, Verbena.

Order II. Diginia.

Antmoxantuujr. Calyx glume 2-valved, 1- 
flowered: corol glume 2-valved, acuminate, awn: ed : seed 1.4. 10-odoratum, (sweet vernal grass) spiked; floret larger than awn. 2f-An American variety, altissimum, is larger and of a darker green. Ires.

* Holcus, Festuca, Saccharum.

\section{CLASS III. TRIANDRIA.}

Order I. Monogynia.

\section{A. Flowers suferior.}

VAleriana. Calyx o, or with an extremely small margin: corol 1-petalled, 5 cleft, base gibbous : seed 1 : stamens, $1,2,3$ and 4 , exsert. 48 . 56-haucifora, (valerian) root-leaves pinnate, stemleaves ternate. $2 \% \mathrm{r}$.

FEDIA. Calyx 3 to 6 -toothed: corol 5-parted: nut 2 or 3 -celled: seed naked, or crowned with a tooth. 48.56-radiata, (lamb lettuce) leaves spatu1ate. $\odot$. b.

IRIS. Calyx, spathe 2-valved: corol 6-parted, divisions alternately reflected: stigmas petal-like, 6. 18. - humila, (dwarf flower-de-luce) scape shorter than the leaves. 2\%. b.-virginica, (wild iris or flag) stem 2-cornered, with alternate leaves. 24.y.b. - gracilis (Boston iris) petals beardless, inner ones erect: leaves linear, flat: germ triangular, twice groved on each side. Bigelow. This plant is very abundant near New-Haven as well as Boston.

\section{B. Flowers inferior.}

Commelina. Calyx, spathe cordate: corol 6 petalled : nectaries 3 , cross-form, inserted on peculiar filaments : capsule sub-globose, 3-celled. 6. 13-communis (day-flower) stem creeping. (2).b. e.

Leptanthus. Calyx, spathe 1-flowered: corol long-tubular; border 6-parted with the stamens on 
the divisions: capsule 3-celled, many-seeded, gaping at the angles. 3. 1-reniformis, leaves roundcordate; spathe. $21 . \mathrm{w}$.

Xyris. Calyx, glume 2-valved, in a head: corol 3-petalled, equal, crenate: capsule 3-valved, many-seeded, 6. 13-ancefis, (yellow-eyed grass) culm 2-edged. 2\%. $y$.

C. Flowers grassy: valves of the calyx glume: like : corol none.

Schoenus. Spikelets sub-convolute, acute : scales heaped in fascicles, outer ones dry, shining: corol 0: seed 1, roundish, naked or surrounded. with bristles. 3. 9-albus, (bog-rush) leaves bristleform. 24-gloneratus, leaves flat. 2s:

Cyperus. Glumes chaffy, scales imbricated 2. ways : seed single, beardless : spikelets compress.ed. 3.9-sfiathaceus, (galingale) axillary racemes. 21-rotundus, umbel doubly-compound. $2 \%$.

Scripus. Glumes chaffy, scales imbricated eve: ry way : seed single, naked, surrounded with hairs or bristles. 3.9-macrostachius, (club.rush) panicie clustered, leafy, terminal. 2 -triqueter, spikelets lateral, sessile, bunched. 21-retrofractus, umbeis simple. 21-acutus, culm round, very tall. 2!.

Eriopionem. Glume chaffy, imbricated every way : seed beset round with very long dense woolly hairs. 3.9-cyherinum, (cotton grass) panicles thrice-compound, one growing from the top of another. 21-angustifulium, leaves channelled, trio. angular at top-polystachium, leaves flat, spikes. peduncled. 24 .

Trichophorem: Calyz, scales imbricated eve. ry way: seed beset with capillary bristles, which at length project out, always 6 in number: spikelets ovatish. 3. 9-erioflhorum, (light-hair) culm leafy: spike crowded.

B 2 
D. Flowers grassy: valves of the calyx slume. like: corol 2-valved.

Spartina. Calyx 2-valved, compressed, one of them keeled and longer than the other: corol 2.valved. 3.9-cynosuroides, (salt-grass) peduncles jough. 24 -juncea, peduncles smooth. $4-5$ labra, valves of caly $\mathrm{x}$ mostly glabrous. 4 .

Cenchrus. Involucre divided, echinate, 3 or A-flowered: glumes 2 -valved, 2 -flowered: corol 2-valved, awnless: style 2-cleft, 4, 10-echinatus (hedgehog grass) spike conglomerate.

* Juncus, Galium.

Order II. Digynis.

The proper Grasses.

A. Flowers nerfect.

ist. Glume 1:flowered.

Oryzopsis. Calyx 2-valved, lax, obovate : $\mathrm{co}$. jol teretish-ovate, leathery: valves 2 , outer one awned at the apex : appendages 2 , linear, chaffy, 4. 10-asperifolia, (mountain rice) leaves rough.

Panicum, Calyx 3-valved, the third valve dor. sal and very minute : corol 2.valved : infloresence various. 4. 10-crus-galli, (cockfoot grass) spike doubly-compound. - -glaucum, (panic grass) spike terete. (0)-verticillatum, spike whorled. ()-lati. folium, leaves broad and hairy at the neck of the sheath. 21-nitidum, panicle very branching, flowers minute.

Digitaria. Calyx 2 or 3-valved, concave; the outer one very small or none, the inmost one of the length of the corol : corol 2-valved, oblongovate, awnless: style very long: spikes digitate, linear : flowcr's in pairs. 4. 10-sanguinalis, (finger grass) culm creeping, flowers imbricate. - dac: tylon, scions creeping, spikes finger-form. 2 . 
ARISTIDA. Calyx 2-valved: corol 1 -valved, with 3 awns at the tip. 4.10-dichotoma, (beard grass) culm forked.

Alopecurus. Calyx 2-valved: corol 1-valved, simple at the tip; sometimes awned at the base. 4. 10-1ratensis, (foxtail grass) culm erect. 2 - seniculatus, culm geniculate.

Phalaris. Calyx 2-valved, valves keeled, nerved, equal in length, including the 2 -valved pilose corol. 4. 10-arundinacea, (ribbon grass) panicle oblong, compact, leaves particoloured. 2\%. e.

Hordeum. Calyx lateral, 2 -valved, 1 or 2 -flow. ered; florets in threes, the middle one sessile, lateral ones often barren: corol 2 -ralved, acute, outer valve awned. 4. 10-vulgare (barley) florets in 2 erect rows. e.-jubatum, involucres bristle-form.

Minium. Calyx 2 valved, 1-flowered, ventricose : corol 2 -valved, very short: stigmas pencilform. 4. 10-effusum, (millet) panicled, awnless. e.

Agrostis. Calyx 2-valved, 1-flowered, valves acute : corol 2-valved : stigmas longitudinally hispid or plumose, (florets spreading) 4.10-vulsaris (red top) panicle spreading, glume calyz twice as long as corol. 2 --alba, panicle las, culm creeping-stricia, panicle long, stiff and strait. 24.

Saccharum. Calyx involucred with long wool, 2-valved: corol 1 or 2-valved: stigmas 1 to 3. 4 . 10--oficinarum (sugar cane) flowers panicled, leaves flat. $2 \downarrow$.

Muhlenbergia. Calyx l-valverl, very minute : corol 2-valved, base hairy, ouicr valve awned at the top: seed 1, oblong acuminate (Flowers panicieu) 4. 10--diffusa, glabrous : cu! in weak : panicle slender, compact. 4 --erecta, pubescent ; culm erect and firm: panicle lax.

Leersia. Calyx 0: corol 2-valved, closed; valves compressed, boatform, awn!ess. 4. 10-ory * 
zcides (cut grass) spikelets spreading. 4 -virgin$i c a$, spikelets close-pressed-lenticularis, spikelets imbricate, glumes ciliate.

Tiric HоDium. Calyx 2-valved, valves nearly equal, acute; keel with small spines : corol l-valved, awnless, shorter than calyx : stigmas nearly sessile, sub-hispid. Panicled. 4. 10-laxiflorum, (thin grass) culm erect, branched, slender. 24.

PhLeum. Caly $\bar{x}$ indurated, 2-valved, sessile; linear, truncate, bicuspidate : corol inclosed. 4. 10. - pratense (timothy grass) spike long, cylindric. 2!. 2d. Glumes 2 or 3-flowered; hanicled.

AIrA. Calyx glossy, 2-valved, 2-flowered : co. rol 2-valved, awnless, or awned at the base. 4. 10. - cespitosa, (hair grass) corol with a strait awnflexuosa, awned, geniculate-obtusata, awnless.

3d. Glumes 2-florvered or more; close-shiked.

Eıymus. General calyx involucre-like, 4-leav: ed, 2-flowered ; partial calyx lateral, 2-valved, aggregate-ternate, many-flowered. (In a spike.) 4. 10-virginicus (lime-grass) involucre striate, long awned. 24.

Eleusine: Calyz awnless, many-fluwered : inflorescence an unilateral digitate spike : corol 2valved, awnless. 4. 10-indica, (dog-tail grass) 3pikelets about 6-flowered.

Limetis, or Dactyis. Calyx 2-valved, one valve smaller: corol 2-valved, awnless, compressed, carinate: style long. Panicles strait; spikes lateral-flowered, florets imbricate. 4. 10-glomerata, (orchard grass) a panicle condensed into a spike-form head. $\boldsymbol{\psi}$.

Secale. Calyx. 2-valved, 2 or many-flowered, opposite, solitary: glumes linear-lanceolate, smooth or channelled both sides. 4. 10-cereale, (rye) glumes rough-ciliate.

TrItICUM, Calyx 2.yalved, about 3-flowered, 
alternate ; florets obtusish and pointed: glumes beardless or interruptedly bearded: spikelets shortish. 4. 10-hybernum, (winter wheat) nearly awnless. ô -aestivum, (summer wheat) long-awned.

Lolium. Calyx 1.leafed, permanent, many-flowered: florets in a 2-rowed simple spike. 4. 10ferenne, (darnel-grass) spike awnless, 4 --temulentum, spike awned. (:).

Atreropogon. Calyx 1-valved, 2-flowered: corol 2-valved, awned. 4. 10-ahludoides (hairy beard-grass) leaves very narrow. $\boldsymbol{\psi} 4$.

4th. Glumes many-flowered; in hanicles:

UNiold. Spikelets flat, 2-edged, ovate : calyx many-glumed: corol 2-valved, awnless, inner one smallest: stamens 1 to 3 : appendages 2, somewhat 2.horned. 4. 10-sficata (sea rush-grass) panicle spike-form, pointing one way. $\boldsymbol{z}$-latifolia, panicle lax. 24. Monandrous.

BRIzA. Calyx 2-valved, many-flowered: spikelets of the panicle 2-ranked: valvelets inflated heart-form, obtuse; inner one minute. 4. 10-canadensis (quake-grass) culm erect, panicle lax.

Sorghus. Polygamous. Florets in pairs; one perfect and sessile, the other staminate or neutral and pedicilled. 4. 10-saccharatum, (broom-corn) panicle somewhat whorled, spreading: seed oval. (i).

PoA. Calyx 2-valved, many-flowered: corol ovate; valves 2 -coloured, acutish, scarious at their margins: spikelets of the panicle ovate, awnless. 4. 10-1tratensis, (meadow-grass) panicle diffused, spikelets 4-flowered. 4 -trivialis, panicle diffused, spikelets 3 -flowered. 2;-compressa, (blue-grass) culm compressed, panicle pointing one way. $21-$ rehtans, culm creeping, spikelets 6 to 10-flowered. ro. 
Festuca. Calyx 2-valved, many-flowered; spikelets ublong, teretish, or diverging; awnless or furnished with a terminal awn. 4. 10-pratensis (fescue-grass) panicle pointing one-way, spikelets linear. $2 i$ - lutior. panicle nodding, spikelets ovatelanceolate 2 -Auitans, panicle erect. 4.

Bromus Calyx 2-valved; spikelets ublong, terete- 2 ranked; valves awned beluw the tip 4. 10 . - secalinus, (chess) awnless, panicle spreadingmullis, (broom-grass) florets imbricate, panicle compact. $\hat{o}$.

Avexa. Calyx 2-valved, many-flowered; valives with a twisted awn on the back : glumes membranaccous and somewhat follicle-like. 4. 10-sativa, (oats) panicled, calyz 2-seeded. (0)

Airuno. Calyx 2-valved, one or many-flowered, glabrous: florets heaped together : corol surrounded with wool. 4.10 - hlhragmites, (reed-grass) calyx 5-flowered. 4 .

\section{B. Flower's nolygamous.}

1st. Glume 1-flowered.

AndRopogon. Calyx glume 2-valved: corol 3valved: flowers in pairs; 1 sessile perfect, the other peduncled, stainate, awnless-rarely neutral. 4. 10-nutans, (beard-grass) panicle compact, nodding, calyx bristly.

2d. Glume 2-flowered, or more. Panicled.

Holeus. Calyx glume 2-valved, opake, nervous : corol smaller than calyx, 2 -valved, the outer one-awned under the tip: nectary linea. 2-parted: stigma subsessile. 4. 10-lanatus, (soft-grass) glumes hairy. 4 -odoratus, glumes 3-flowered, pointed, awnless. 24.

* Alsine. 


\section{Order ili. Trigynia.}

A. Corol 5-petalled.

Hozosteum. Caly \& 5-leavtd: capsule 1-celled, nearly cyliudric, bursting at the top.22. 82-succulenium, (succulent chickweed) leaves oval, fleshy:

B. Corol 3-petalled.

LECHEA. Calyx 3-1caved : capsule 3-celled, 3 valved, with 3 inner valvalets: sted 1.22.8:major, (pinweed) flowers dull, scattered-minor, flowers dull, panicled.

\section{Corol none.}

Mollugo. Calyx 5-leaved, inferior: capsule 3-celled, 3-valved, many-seeded. 23.82-verticillata, (carpet-weed) leaves whurled, stern prostrate. (i). W.

Queria. Calyx 5 leaved, inferior : capsule 1celled : seed 1. 22.82-canadensis, stem forked, leaves lance-ovate. 2 .

Proserpinaca. Calyz 3-parted superior: nut 3-sided, 3-celled, crowned by the calyx. 15.22halustris, (mermaid weed) leaves lanceolate, serrate above, and divided below, water. 2!.g.

* Zanthoxylon, Alsine.

\section{CLASS IV. TETRANDRIA.}

Order I. Munugyia.

A. Flowers 1 heiallid, inferior, calyx 4.ciefi.

Caluicalipa Corol 4-cleft tubular: berry 4seedec. 43.38-Americana, (Bernuda nulberiy) cymes sessile, leaves oval serrate . $h$.r.

Plantago. Corol 4-cleft reflexed: capsule 2celled, opening transverseiy : strmens very long. 54. 31 -inajor, (planta!n) leaves broad ovate 4 . w. -lanceclata, (ribwort) leaves lanceolate. 24.

Lycrum. Corol tubular, having the throat closed by the beards of the filaments: berry 2.celled, 
many-seeded. 28. 41-carolinianum, (box thorn) leaves oblong-spatulate, sharp. $h$. p.

Frasera. Corol 4 cleft. spreading; segments with bearded glands in the middle : capsule compressed, sub-margined, 2-valved : seeds few, imbricated-qualteri. (pyramid flower) leaves oblongoval, opposice and whorled. y.

Bartonia, or Centaurella. Corol 4-cleft, bell-form, permanent: capsule 1-celled, 2-valved, many-seeded-Paniculata, (screw-stem) stem square. w.

Houstoxia. Corol salver.form: capsule 2celled, 2-valved, 2-seeded. 47.57-coerulea, (Venus' pride) root-leaves ovate. 4 .b.

B. Flowers 1-theialled, sunerior.

Mitchella. Calyx 4 -toothed: corols 2 on each germ, tubular: berry double, 4 -seeded : stigmas 4. 4.8. 57-rehens, (partridge-berry) stem decumbent, leaves roundish. $\%$. w.

SANGuiscrba. Calyx inferior, 2-leaved: corol superior, 4 parted: capsule between the caly $\mathrm{x}$ and corol. 54.92-medica, (burnet saxifrage) stamens longe: than colol, spike cylindric. $y$.

Cephalanthus. Inflorescence in a head: general caly $\mathrm{x}$ none: proper calyx superior : corol funnel-form: receptacle globular, hairy: capsules 2 to 4-partible: seed solitary, oblong. 48.57-occidencalis, (button bush) flowers in heads. h.w.

Linnaea. Calyx double; that of the fruit 2. leaved, that of the flower 5.parted: corol bellform: stamens somewhat didyriamous: berry 3 . celled, dry. 48.58-borealis, stem creeping, flowers in twins. $h$, w. r.

Rubia. Calyx \&-toothed: corol 4 or 5-cleft, bell-form : berries 2, one-seeded, (stamens 4-5; leaves stellate.) 47.57-tinctoriu, (madder) leaves whorled, stem square, prickly. $21 \cdot y$. 
Gaziux. Calyx 4-toothed: corol flat: fruit dry : seeds 2 , roundish : (leaves stellate.) 47.57tinctorium, (bedstraw) stem whorls in sixes, branch whorls in four's. $4 . w$-aharine, fruit prickly, whorls in eights. (-w.-verum, fruit smouth, whorls in eights. 4.y.-brachiatum, whorls in fours. 4.w.-asfirellum, whorls all in sixes, stem decum. bent. $4 . w$.

Dipsaces. Common calyx many-leaved; proper calyx 1-leafed superior: receptacle chaffy : seed 1, crowned: flowers aggregate. 48.56-fullonum, (teasel) chaff hooked. ô. c.-sylvestris, chaff strait. $\hat{o}, b$.

\section{Flowvers 4-hetalled, inferior.}

Ammania. Calyx tubular, plaited, 8-toothed: petals 4 , or none, on the calyx : capsule 4 -celled -(sometimes 2-celled and 8-stamened.) 17.91humilis, (tooth cup) stem procumbent. $\odot . r$.

Corsus. Calyx 4-toothed: drupe with a 2-celled nut. Some species have a 4-leaved involucre. 45. 58-florida, (dogwood) involucres large, petal-like $h . y$. w. haniculata, cymes panicled. $h$. w.-alba, cymes flat. 21. w.-circinata, leaves orbicular, acuminate-branches worty.

LUDWIGIA. Calyx 4-parted, the divisions long, permanent: capsule 4-cornered, 4.ceiled, perforated at the top, many-seeded-(petals sometimes wanting.) 17.8S-alternifolia, (seed-box) glubrous. (-).y.-tilosa, rough-haired. 4.y.

E. Flowers not comfilete.

Pотноs. General calyx a spathe: perianth o: spadix simple, covered with flowers: petals 4, wedge-form : berries globose, 2 -seeded, inclosed in a spongy receptacle. 2. 7-foetida, (skunk-cabbage) stemless, leaves oval if p.

Isyardia. Calyx bell-form, 4-parted, superior:

C 
corol o: capsule 4-celled, 4-cornered, many-seeded, surrounded by the calyx. 17. 91-palustris, (water-purslane) creeping, leaves ovate. (-) $g$ •

Rivina. Corol 4-petalled, inferior, permanent: calyx o: berry 1-seeded: seed lenticular, rough, (stamens 4,8 or 12) 12.29 -laevis, racemes simple, leaves ovate. $r$.

* Convallaria, Cardamine, Prinos.

\section{Order II. Digria.}

HAMANELIS. Involucre 3-leaved: perianth 4leaved: petals 4 , very long, linear : nut 2 celled, 2-horned. 54. 78-virginica, (witch hazel) leaves undulate-toothed, ob-ovate. Flowers in autumn, and perfects its seed the following spring. $h \cdot y \cdot$

* Cuscuta, Swertia.

Order iv. Tetragyia.

Irex. Calyx 5-toothed; corol 4-parted, wheelform; style o; berry 4-celled, cells 1 -seeded. 43. 95-opaca, (holly) leaves evergreen, repand, spinous. h.g.-canadensis, leaves deciduous, ovate, mostly entire. h.w. y.

SaGina. Calyx 4-leaved; petals 4; capsule 4celled, 4-valved, many-seeded. 22.82-12rocumbens (pearl-wort) stem procumbent. 2 .w.

Potamogeton. Calyx o: petals 4 : style o: seeds 4. 15. 6.-natans, (pond-weed) upper leaves with rouncled bases-fiuitans, upper leaves lance-oval.

\section{CLASS V: PENTANDRIA.}

\section{Order I. Monoginia.}

A. Flowers 1-hetalled, inferior; having 4 naked seeds. Rough-leaved plants.

Pulmonaria. Calyx prismatic 5-angled: corol funnel-form, with an open throat: sceds roundish, obtusc. 41.42-virginica, (lungwort) leaves ovate, panicle terminal. 
Lithospermum. Calyx 5-parted: corol funnelform, with an open throat: seeds ovate, pointed, stoney: stamens and pistils inclosed. 41, 4.2-officinale, (stone-seed, or gromweli) seeds smooth. $\boldsymbol{2}$. $y$-arvense, seeds wrinkled.

Cynoglossum. Calyx 5-parted : corol funnelform, vaulted, throat closed : sceds depressed, affixed laterally to the style within. 41.42-officinale, (hound-tongue) leaves sessile. 2 \%.p.-amplexicaule, upper leaves clasping. 24.w. b.

Anchusa. Calyx 5-parted: corol funnel-form, vaulted, throat closed: seeds marked at the base, and their surface generally veined. 41. 42-officinalis, (bugloss) spikes one-sided, leaves lanceolate. h.e.

Mrosotis. Calyx half-5-cleft: corol salverform, curved, 5-cleft, vaulted, throat closed, the lobes slightly emarginate: seeds smooth or echinate. 41.42-scorhioides (scorpion grass) racemed, leaves lance-oval. 24 . b.

Heliotropium. Calyx tubular, 5-toothed: corol salver-form, 5 -cleft, with teeth or folds between the divisions; throat open. (Spikes recurved, involute.) 41.42.-indicum (turnsole) leaves heartovate, acute. (b. e.

Onosmodium. Calyx deeply 5-parted: corol oblong, somewhat bell-form, with the acute divisions converging, the upper pari of the corol being ventricose and half-5-cleft, throat open: anthers sessile enclosed: styles exsert. (Spikes revolute.) 41. 42-hisfidum, (false gromwell) leaves ovalo lanceolate, papillose-punctate.

BORAGO. Corol wheel-form, the throat closed with rays. 41: 42-officinalis, (borage) leaves alternate. e.

SrMphitum. Limb or upper part of corol, tubu. lar-swelling, the throat closed with subulate rays. 
41. 42-officinalis, (comfrey) leaves decurrent. 4 . w. e.

Echrum. Corol mostly irregular, throat naked, large: stigma cleft. 41. 42-vulgaris, (viper's bugloss) stem bristly, spikes lateral. b.

B. Flowers 1 hetalled, inferior; seeds covered with a 1-celled capsule : calyx 5-parted or 5-toothed.

Hydrophylum. Coral bell-form, 5-cleft, with 5 longitudinal honey-bearing groves inside: capsule globose, 2-valved: stigma 2-cleft. 41.42virginicum, (water-leaf) leaves pinnate-pinnatifid: 4. w.-canadense, leaves lobed. 4.w.

ANAGALLIS. Corol wheel-form, deeply 5-parted : capsule opening transversely : stamens hairy. 20. 34-arvensis, (scarlet pimpernel) stem square, procumbent; leaves dotted. (). r.

Lysimachra. Coral wheel. form : capsule globulal, 10-valved, mucronate: stigma obtuse. (In some species the filaments are united at the base.) 20. 34-ciliala, (loosestrife) petioles ciliated. 4. y. thyrsifolia, racemes lateral. $2 \%$ - -liybrida, leaves opposite, lanceolate, acute at the base-stricta, stem squarer, racemes terminal 4. y.-quadrifolia, leaves whorled or in pairs nearly opposite, with axillary 1 -flowered peduncles. 24. $y$. Near the outlet of the Beaver Pond (New-Haven) I found this specics in abundance, with $2,3,4,5$ and 6 leaves together'; though Linneus allows but 4 .

Dodecantheon. Corol wheel-form, reflexed capsule oblong: stamens on the inside of the tube : stigna obtuse. 20. 34-meadia, (false-cowslip) Ieaves repand-toothed, umbels many-flowered. 4 .p.

Menyanthes. Corol hairy : capsule ovate: stignıa 2-cleft. 21. 34-trifoliata, (buck bean) leaves 3 on a petiole. 4 . w.

PRIMULA, Umbellets involucred: tube of corcl 
cylindric, throat open, divisions of corol emarginate : capsule 1-celled with a 10-cleft mouth : stigma globular. 21. 34-vulgaris, (primrose) scape 1flowered. 4.-veris, (cowslip) scape many-lowered. 4.

Hotтonia. Corol salver-form: stamens on the tube of the corol: stigma globular. 21. 34-fialustris, (feather-leaf) flowers whorled. 4 .

\section{Differ from the above in having 2-celled cansules.}

Spigeita. Corol funnel-form: twin capsules 4valved, 2-celled: stigma simple: seeds many. 47. 46-marilandica, (pink-root) leaves lance-ovate, sessile, opposite. 4. p.

Convolvulus. Corol funnel-form, plaited: stigma 2-cleft or double: cells of the capsule 2 or 3 ; each 2-seeded. 29.43-setium, (morning glory) hind lobes of the arrow-form leaf truncate. 4 . p. w.-stans, stem erect. 4. w.-batatas, (sweet potatoe) leaves heart-halbert-form. $\quad$ ll. p. w.-tricolor, leaves lance-ovate.

Datura. Calyx tubular, angled, caducous: corol funnel-form, plaited: capsule 4-valved, 4-celled, smooth or thorny. 28. 41 -stramonium, (thornapple) pericarp spinous. $)$.w. b.

Nicotrana. Corol funnel-form, limb plaited: stigma notched : stamens inclined: capsules 2celled, 2-valved. 28:41-tabacum, (tobacco) leaves decurrent. $\bigcirc$. p.

Verbascum. Corol wheel-form, somewhat ir. regu!ar: stamens declined, hairy : capsules 2 . celled, 2-valved; valves inflexed : many-seeded. 28. 41.-thahsus, (mullein) leaves decurrent. (-). $y$. -blattaria, (cockroach mullein) leaves clasping. 今. $\mathrm{y}$.

Chirona. Calyx somewhat bell-form: corol C 2 
salver-form or wheel-form : anthers becoming spiral; filaments on the tube of the corol : pistil de. clined : pericarp 4-celled. 20. 40-angularis, (centaury) leaves clasping, stem acute-angled. 9 . p. -venosa, stem somewhat decumbent, leaves lance. olate. 4. w.

Atropa. Corol bell-form: stamens distant: berry globular, 2-celled, sitting on the calyx. 28 , 41-belladonna, (deadly nightshade) leaves ovate. 2. e.

Fhacelia. Corol somewhat bell-form, 5-cleft : stamens exsert : capsule 2-celled, 2 -valved, 4 -seeded. 41.42-finbriata, corol fringed. (-) b. - har. viflora, corol entire.

Hroscyanus. Corol funnel-form, obtuse, irregular: stamens inclined : capsule 2-celled, covered with a lid. 28.41-niger, (henbane) leaves clasping, pinnate. $\delta \cdot y \cdot$ p. e.

\section{Differ from the above in havins 3.celled capsules.}

Diafensia. Calyx 5-leaved, imbricated with leafets : corol salver-form; limb flat, 5-cleft: stamens crowning the tube of the corol: capsule 3-valved, many-seeded. 21.43-lafnponica, leaves linear. 24. w.

Phlox. Calyx prismatic : corol salver-form, with a tube somerwhat curved: filamenis unequal in length: stigma 3-cleft : capsule 3-celled, 1-seeded. 20. 44-fzaniculata, (lichnedia) segments of corol rounded. 24. p.-subulata, lobes of corol notched. 24. w. p.

Polemoxium. Calyx-half 5-cleft : corol wheelform, 5-parted: stamens on 5 valves closins the bottom of the tube: stigma 3-cleft. 29.41-rep. tans, (Greek-valerian) leaves pinnate, root creeping. $4 . b$. 
IPONOEA. Corol funnel or bell-form, with 5 plaits : stigma globe-headed, papillose: capsule 2 or 3-celled, many-seeded. 29. 13-quumoclit, (cypress vine) leaves pinnatifid. (-) p. $r$.

Pyxidanthera. Divisions of calyx lying over each other, chaffy-membranaceous : corol bellform, 5-parted: filaments lamellar : cells of anthers opening transversely: stigmas 3 : style thick. 21 . 13-barbulata, (box-flower) leaves lance-wedgeform. $h$. w.

Differ from the above in having 5-celled cafisules.

Azalea. Corol tubular, half-5-cleft, somewhat oblique: stamens on the receptacle : stigma obtuse, usually ending with 5 short papillae. 18.50viscosa, (wild honey-suckle) tube of corol twice as long as the divisions. $h$. w. - nitida, tube of corol not twice as long as the divisions. $h$. w. $y$.

Vinca. Corol salver-form, twisted, border 5. cleft, with oblique divisions; throat 5 -angled : seed naked, oblong: follicles 2, erect, terete, narrow. 30. 47-minor, (periwinkle) stem procumbent, lance-oval.

Differ from the above in having 2-celled ber. ries.

Soraxum. Calyx permanent: corol bell or wheel-form, 5-lobed, plaited: anthers thickened, with 2 pores at the top: berry containing many seeds. 28. 41-tuberosum, (potatoe) leaves interruptedly pinnate. 2\%.w. p.-nigrum, (nightshade) lcaves angular. w.-dulcamara, (bittersweet) climbing, leaves halbert-form. $h . b$.

Physalis. Corol bell or wheel-form : anthers converging : berries contained in an inflated calyx : seeds numerous. 28.41-alkekengi, (winter-cherry) leares in pairs, entire, acute. 
CaPSICUM. Corol wheel form : berry juiceless : anthers converging : caly $x$ angular. 28. 41 -annuu $m$, (red pepper) flowers solitary. O.w. e.

C. Flowers 1-pletalled, half-inferior: calyx 5-cleft.

Samolus. Corol salver-form, 5-lobed with intermediate scales fencing the stamens between them : capsule 1-celled, 5-toothed; many-seeded. 21. 34-valerandi, (brookweed) leaves obtuse, racemes many-flowered. 24.w.

D. Flowers 1-petalled sunerior: calyx 5-clefi.(Stems woody.)

LoNICERA. Corol tubular, 5-cleft, unequal : berry 2 or 4 -celled : seeds inany. 48.58-sempervirens, (trumpet honey-suckle) tube of corol dilating. upwards. h.r. e.-dioica, corols ringent, gibbous at the base. $2 . y \cdot \mathrm{p}$.

Xylosteum. Corol tubular, border 5-parted, nearly equal : berries in pairs, whose bases are connate, or single coadunate; 2-celled. 48.58ciliatum, (fly honey-suckle) peduncle 2-flowered, berries distlnct; leaves entire, hairy. $h \cdot y \cdot w \cdot-$ solonis, two flowers seated on one germ; berry. clouble, not distinct: leaves oblong-ovate, villose. Found on the White Mountains, (N. H.) by Dr. D. Solon. C. H. Smith.

Diervilla. Calyx oblong : corol twice as long as calyx, funnel-form, border 5-cleft, spreading : capsule oblong 4-celled, many-seeded. 48. 58-canadensis, (yellow honey-suckle) leaves ser: rate: $h: y$.

\section{(Stems not ryoody.)}

Campanula. Corol bell-form, closed at the bot: tom by valves bearing the stamens : stigma 3 to 5 cleft: capsule 3 to 5 -celled, opening by lateral pores. 29. 52-kerfoliata, (bell-flower) leaves clasping. 
ish-cordate, stem-leaves linear. 21. b.-media, (Canterbury bells) capsules 5-celled, flowers erect. s.

Lobelia. Corol irregular, often irregularly slitted: anthers cohering and somewhat curved : stigma simple : capsule 2 or 3-celled: 29.52-cardinalis, (cardinal flower) spike pointing one-way. $\Psi$. r.-inflata, capsules inflated. $\hat{\delta}$.w. b. - hallida, leaves toothed. 24. w. b.- sythilitica, leaves with sinuses reflexed. $2 \%$ b.

Miraeilis. Corol funnel-form, coarctate below : caly $\mathrm{x}$ inferior : germ between the calyx and corol : stigma globular. 54. 32-jalaha, (four o'clock) flowers peduncled, leaves glabrous. e.

Trinsteum. Calyx of the length of the corol: corol tubular, 5-lobed : berry 3-celled, 3 seeded.48. 58- herfoliatum, (feverwort) leaves connate; flowers whorled. 2\%. p.

E. Flowers 5-hetalled, inferior. (Stems woody.)

Rhamnus. Calyx cup-form, 4 or 5-cleft; corol consists of scales fencing in the stamens and inserted on the calyx (sometimes wanting); stigma either simple, 2 or 4 -cleft; berry 3 or 4 -seeded; (sometimes polygamous or dioecious.) 43.95.catharticus (buck-thorn) spines terminal. $h$-alnifolius, no spines. h.

Cenvothus. Petals bay-like, vaulted, standing in the cup-form caly $\mathrm{x}$; berry, or capsule, dry, 3grained. 43, 95.-americanus (New-Jersey tea) leaves oval, serrate, downy beneath. h.w.

Cenastrus. Calyx flat: corol spreading : capsule 3-angled, 3-celled, berry-like : stigma 3-cleft : seedls calyptred. 43, 95--scandens (staff tree) climbing : fruit berry-like, red, permanent, opening with 3 -valves. $h$. w-y.

Euonysus. Calyx 5-parted, flat: corol flat: 
capsule 5-angled, 5-celled, 5-valved, coloured : seeds calyptred. 43.95-atropurfureus (:pindle tree) peduncles many-flowered. $h$.p.-americanus, peduncles about 3-flowered. $h$. y.

VIris. Calyx 5-toothed: petals cohering at the tip hood-like, withering: berry 5 -seeded, globular. (Often dioecious.) 46.72-vulhina (grape vine) leaves heart-form, tooth-serrate. h-rifiaria, leaves short, 3-cleft, petioles hairy. h--rotundifolia, leaves heart kidney-form. h.

AMpelopsis. Calyx 5 -toothed: petals reflexed, spreading: berry 5-seeded, globular. 46. 72quinquefulia (false grape) leaves in fives. $\eta_{2} . g$.

ITEA. Calyx 5-cleft, bearing the 4 lorg linear reflexed petals: stigma capitate, 2 -lobed : capsule 2 -valved; the valves bearing the seeds ant having inflexed margins. 18. 50-virginica, leaves ovate, serrate; racemes terminal. $\eta_{0} . w$.

(Stems not woody.)

Impatiens. Calyx 2-leaved: corol irregular spurred : anthers cohering: capsule elastic, 5 -valved. 24. 73-nolli-me-tarigere (jewel weed) perluncles many-flowered. O.y.-balsamina (touch-menot, or balsam weed) peciuncles 1 -flowered.

VIola. Calyx 5-leaved: corol irregular, with a horn behind (sometimes the horn is wanting:) anthers attached by a membranous tip: capsule 1celled, 3-valved. 29. 80.-stemless. Cucullata, (violet) leaves cordate, flowers inverted-lanceola$t a$, leaves lanceolate-falmata, leaves palmatejeciata. leaves pedate-frimulifolia, leaves heartoblong, petioles membrane-winged. STEMmedPubescens, leaves cordate, hairy-canadensis, leayes cordate, glabrous-tricolor, leaves tooth-crenate. e.

Craytonia. Calyx 2-valved: stigma 5 -cleft: capsule 1-celled, 3-valved, 3-sceded. 13.86-virginica, leaves lance-linear. $2 !$. 


\section{CLASS V. ORDER II.}

\section{F. Flowers 5-hetalled, superior.}

Ribes. Corol and stamens inserted on the ca. lyx: style 2-cleft: ierry many-seeded. 36. 85rubrum (currant) flowers flatish. h. g.e.-nigrum, flowers oblong. $h \cdot g-y$-fortidum, Howers cylindric. h. w-y.

Hedera. Petals oblong: berry 5-seeded, surrounded by the caly $\mathrm{x}$ : style simple. 45.58 -helix (European ivy) leares 5 -angled flower's umbelled. h. e.

G. Corols quanting.

Graux. Calyx inferior, 5-lobed, coloured: cap. sule 1-celled, 5-valved, 5-seeded, surrounded by the calyx. 17.91-maritima (sea-milkwort) grows in salt water. 4 .

Thesium. Calyx superior, half-5-cleft, bearing the stamens : nut 1 -seeded, covered with the per. manent calyx. 3i 24-umbellatum (false toad-flax) leaves oblong. 24. w-g.

\section{Order II. Digynia.}

A. Follicles 2: corol 1-pietalled, suith a 5-fiarted border.

Periploca. Nectary pitcher-form, putting out 5 threads, surrounding the stamens : corol wheelform: stigma capitate, 5-cornered 30. 47-graeca (follicle vine) flowers terminal. 2\%. p.

Apocyum. Corol bell-forin : stamens alternating with 5 filamentous pointed nectaries: stigma broad almast sessile : follicles long linear. 30.47 -androsemifulium (dog-bane) cyned, corol spreading. 24.-cinnasinum, (Indian hemp) panicled, corol erect. 4 y-g.

B. Cafisules 1 or 2-celled, 2-valved: corol 1-hetalled : calyx 5-cleft.

SWERTIA. Corol wheel-form with nectariferous 
pores at the bise of the divisions: capsule 1-celled. 20. 46-fiusilla. (false gentian) stem 1 -flowered. (.).b.-corniculata, corol 4-horned.

Gentrans. Corol with a tubular base, without pores: capsule 1-celled, oblong: columellas 2, longitudinal. 20. 46-aponaria. (gentian) corols 5cleft. $24 \mathrm{~b}$-crinita, corols 4 -cleft, tetrandrous. b.

Cuscura. Corol 5-cleft: capsule 2-celled, dividing transversely at the base : seeds binate. 29. 43-americana (dodder) stem twining, leafless, yellow, parasitic. $w$.

\section{Corol 5-fietalled.}

Hevcrera. Calyx inferior: corol on the calyx : capsule 2-beaked, 2 celled. 13. 84-americana (allum-root) leaves radical, cordate. 4 . r.

PA.NAX. Polygamous. Umbelled. Involucre many leaved : calyx 5-toothed, superior: berry heart-form, 2-seeded : caly $x$, in the staminate flowers, entire. 46. 59-quinquefolia, (ginseng) leaflets quinate 4 . g.-trifolia, leaves ternate. 21. W.

D. Corol 0. (Stem herbaceous, except Atrifllex halimus.)

SAzsorA. Calyx 5-leaved with a capsular base: capsule 1-celled, with a spiral seed. 12.29-kali, (salt-wort) thorny, flowers axillary.

Chenopodrum. Caly y 5-leaved, 5-angled, inferior: seed 1, lens-like, invested by the calyx. 12. 29-album, (pigweed) leaves deltoid, erose. (-). g: -rubrum, leaves heart-deltoid, sinuate-toothedhybridum, leaves cordate, tooth-angled-botrys (oak of Jerusalem) leaves sinuate-pinnatifid. - . anthelminticum (wormseed) leaves ovate-oblong, toothed. $\mathrm{g}$.

BETA. Calyx 5-leaved: seed kidney-form within the fleshy substance of the base of the calyx. 12 . 
29-vulgaris (red beet) lower leaves ovate. $\{$. e. - cicla, (white beet) leaves ternate. $\widehat{\delta}$. e.

Atriplex. Calyx 5-leaved, inferior: style 2parted: seed 1, depressed. Pistillate flowers are intermixed, in which the calyx is 2-leaved. 12.29 - hatula, (orach) stem procumbent. - - halimus, stem erect, very branching. $h$. (Stem woody.)

Unmos. Calyx bell-form withering, border 5cleft: seed 1, enclosed in a flat membranaceous samara. (Stamens vary from 4 to 8.) 53.99-americana, (elm) flowers pedicelled; a variety, fiendula, has drooping branches. h. - fulva, (slippery elm) flowers sessile.

Celtis. Polygamous. Calyx 5-paited : styles thickish, spreading: drupe l-seeded: staminate flowers beneath the perfect ones, with 6-parted calyxes t: stamens 6. 53. 99-occidentalis (nettlc tree) leaves ovate rough.

E. Plants umbelliferous. Flowers 5-petalled, 2-seeded.

With general and hartial involucres.

ERyngium. Involucres many-leaved: flowers in heads, all fertile : receptacle chaffy: fruit crowned, 2-partible. 45.60-maritimum (sea-holly) leaves radical spinous. b.

Corrandrum. Coro!s radiate, with petals inflected, emarginate : general involucre 1-leafed; partial one halved: fruit globular. 45.60-sativum, (coriander) fruit globose. w. e.

HYDROCOTYLE. Umbels simple and very small : flowers all fertile: involucre 4-leaved : petals $e \mathrm{n}$ tire : fruit half-orbicular, compressed. 45. 60americana, (water-navelwort) leaves crenate, reniform. w.

Sanicula. Flowers of the disk abortive: umbels crowded into head-like heaps : seeds prickly. 
45. 60-marilandica, (sanicle, blackroot) forked, leaves about 7 -lobed. $\delta$.w.

Heracleum. General involucre caducous : flowers unequal : petals inflexed, emarginate : fruit oval, compressed, striate, margined, emarginate. 45.60-lanatum, (cow parsley) leaves ternate, woolly beneath. 21 . w.

Daucus. Involucres pinnatifid : flowers somewhat radiate, those of the disk abortive : fruit his. pid with hairs. (Umbels funnel-form.) 45.60carota, (carrot) seed bristly. o. w. e.

Angelica. Petals incurved : corols equal : calyx 5 -toothed: style reflexed: fruit roundish, solid, 3-winged both sides. 45. 60-triquinata, (angelica) terminal leafet sessile. 24.

Sison. Petals lanceclate, inflected : umbels with few peduncles: fruit ovate, striate : involucres about 4-leared. 45. 60-ccnadense, (honewo t) umbel irregular, leaves ternate. 2\%.w.

FERUla. Petals heart-form: fruit oval, flatcompressed, with 5 lines on both sides: flowers uniform, all fertile : general involucre caducous, partial ones many-leaved. (Generally tall.) 45.60 - villosa, (giant fennel) leaves triternate, leafets serrate. $2 ! \cdot \mathrm{w}$.

Ligusticum. Involucre membranaceous: calyx 5-toothed: petals equal, involute, entire: fruit 3 or 5 -ribbed each side, oblong. 45.60 -levisticum, (lovage) leaves manifold, upper one irregularly cut. 24. e.

Aмm1. Involucres pinnatifid: corols radiate, all perfect: fruit smooth or striate: umbels lax. 45. 60-cafillaceum, (bishop weed) leaves capillary, many-cleft.

Sium. Involucres many-leaved : petals heartform: fruit somewhat ovate, striate, compressed. 45. 60_latifolium, (water parsnip) leaves pinnate umbels terminal. 2 . w. 
Selixum. Involucre reflexed : perianth entire : petals heart-form, equal : fruit flat-compressed, striated in the middle, oval-oblong. 45.60-canaclense, (milk-parsley) glabrous, leaves doubly-pinnate.

Coxium. Partial involucres halved, somewhat 3-leaved : fruit somewhat globular, marked with 5 lines, crenate at both ends. 45.60-maculatum, (poison hemlock) stem spotted, leaves tripinnate. $\hat{\delta} \cdot w$.

With a fiartial, and ruithout a general, involucre.

Chaerophyles. Involucre concave, reflexed, 5 to 8-leaved: petals infexed, heart-form: fruit oblong, smooth or striated, very glabrous. 45. 60-claytoni, (sweet cicely) petioles 3-parted, leafets pinnatifid-lobed. $2 \%$

Cicuta. Involucre 3 to 5-leaved: fruit subovate, grooved. 45.60-maculata, (cowbane) stipules 2-lobed.

Imperatoria. Fruit roundish, compressed, gibbous in the middle, bound at the margin with a wing: petals inflex-emarginate. 4.5.60-ostruthium, (master-wort) leaves biternate. e.

Scaxdix. Umbel simple: flowers rayed: in. volucre many-leaved, emarginate, or gash-pinnate: fruit somewhat hairy, terminated with a very long beak. 45. 60-cerefolium, (chervil) leaves tripinnate. 1 . w.

Withouit a fiartial involucre, and rarely hav. ing a general one.

Smyrnium. Fruit somewhat compressed, giljbous, striate: petals acuminate, ketled.-(Fruik becoming black.) 45.60-auruem, (alexanders) leaves pinnate. l. $_{\text {y. }}$-integerrimum, leaves tcr. thate or biternate. $h . y$. 


\section{CLASS V. ORDER III.}

Pastinaca. Fruit oval, flat-compressed : petals involute, entire. 45. 60-sativa, (parsnip) leaves pinnate. (3. y. e.

ANEтним. Fruit ovatish, compressed, striate: petals involute, entire. 45.60-foeniculum, (fennel) fruit ovate. 24. e.-graveolens, (dill) fruit flattened. e. e.

A prum. Fruit ovate, striate : involucre 1-leafed : petals equal. 45. 60-graveolens, (celery) stem-leaves wedge-form. $\delta$. w. e.

CARUM. Fruit ovate-oblong, striate : involucres about 1-leafed : petals keel-form, inflex-emarginate. 45. 60-carui, (caraway) stem branching. t. e.

* Polygonum, Scleranthus, Asclepias and Cynanchum, are usually placed here; but they are carried to the 19 th class on the authority of Doct. Smith.

Order III. Trigynia.

A. Corols superior, 5-cleft.

VIBURNum. Calyx 5-parted : berry or drupe 1seeded. 43.58-opulus, (snowball) leaves S.lobed, petioles glabrous. h.-acerifolium, (naple-leaf) leaves 3-lobed, petioles hairy. h.-lentago, (sheepberry,) leaves ovate, acuminate. $h$.

Sambucus. Calyx 5-parted: berry 3-secded. 43. 68-canadensis, (black-berried clder) cymes 5parted. h. w.-hubescens, (red-berried elder) somewhat racemed. $\zeta$. w.

B. Corols inferior, 5-hetalled.

Staphylea. Calyx 5-parted: capsules inflated, connate : nuts globular, having a cicatrice. 23. 95. - trifoliata, (bladder nut) leaves ternate. $h$. w.

Rhus. Calyx 5-parted: berry 1-seeded. 43. 94-cofallinum, (sumach) petioles winged. h2.vernix, (poison sumach) glabrous panicle few-flowered: h.-glabrum, glabrous, panicle dense. 
-tyłhïnum, hairy. $h$-radicans, (poison ivy) parasitic. $h$.

Sarothra. Calyx 5-parted : capsule 1-celled, 3.valved, coloured. 20. 82-gentianoides, (pineweed) leaves minute, close-pressed. y.

Alsixe. Calyx 5-leaved : petals equal: capsule 1-celled, 3-valved, many-seeded; having a free central columella. 22. 82-media, (chick-weed) stem procumbent, leaves long-ovate. (-).

* Euphorbia.

\section{Order IV. Tetragyina.}

Parvassia. Calyx 5-parted: corol 5-petalled: nectaries 5 : heart-form, ciliate, with globular tips: capsule 4-valved, 2-celled : seed membranaceousmargined. 14. 64. 24.-caroliniana, (parnassusgrass) leaves radical, roundish._asarifolia, radical leaves kidney-form. $4 \%$

\section{Order V. Pentagynia.}

ArRita. Umbellets involucred : perianth 5. toothed, superior: petals 5 : berry crowned, 5 celled; cells 1 -seeded. 46.59.-LEAVES TERNATE -racemosa, (spikenard) stem branching. $\psi^{2}$ w. nudicaulis, (wild sarsaparella) scape with umbels. 21. W.-LeAves pinNate-hishida, umbelled. W. -stinosa, panicled. $h . w$.

Linum. Calyx 5-leaved : corol 5-petalled : capsule 5 -valved, 10 celled : seeds solitary : filaments thickening at the base. 14.73-usitatissimzum, (flax) calyx-leaves 3-nerved. ical leaves ovate. $y$.

Drosena. Calyx 5-cleft: corol 5-petalled : capsule 1 -celled, 3 to 5 -valved at the top, seeds many. (Soinerimes 6 -styled. The leaves are always beset witi glandular hairs resembling dew.) 14.64 - rotundifolia, (sundew) leaves orbicular. W.-longifolia, leaves obovate. 
S'ratrice. Calyx 1-based, entire, plaited, scarim ous, inferior: corol 5 -petalled: seed 1. 48.33limonium, (marsh-rosemary) leaves obovate, mucronate. $24 . \mathrm{b}$.

* Spergula, Cerastium.

\section{Order XIII. Polygynia.}

Xanthorhiza. Calyx o: petals 5 : nectaries 5 , pedicelled: capsules 1 -seeded, about 5 in number. 26. 61-afrifolia, (yellow root) leaves pinnate. $h$.

\section{CLASS VI. HEXANDRIA.}

Order I. Monogyni.

A. Flosver with a herianth and corol, without a shathe.

Tradescantia. Calyx inferior, 3-leaved: corol 3-petalled : filaments with jointed heads : capsules 3-celled. 6. 13-virginica, (spiderwort) leaves lanceolate, umbels terminal. $2 \%$. b. e.

Berberris. Calyx inferior, 6-leaved: petals 6 , with 2 glands at the claw of each: style 0 : berry 1-celled, 2-seeded. (Stigma navelled: stamens spring up on being irritated.) - canadensis, (barberry) branches dotted, thorns in threes, flowers racemed.

Caulophylum. Calyx inferior, 6-leaved: petals 6, opposite to the leaves of the calyx: drupe 1-seeded: anther 2-celled, gaping at the margin. 24. 78-thrulictroides, (poppoose-root) leaves more than decompound. $24 \cdot \mathrm{y} \cdot \mathrm{g}$.

Prinos. Calyx inferior, 6-cleft: corol wheelform, 6-cleft ; berry 6-seeded. 43. 95-verticillatus, (winter-berry) leaves obovate-lanceolate, acuminate. (Berries red, permanent.) $h$.w.-glaber? leaves wedge-lanceolate, obtusish, glabrous. $h$.

Cleome. Caly $x$ 4-leaved : petals 4 , ascending: glands 3 , one at each sinuate division of the calyx 
except the lowest: stamens from 6 to 20 , or more : capsule silique-like, 1-celled, 2-valved. Tetradynamous. 25. 64-dodecandra, (faise.mustard) leaves ternate, stamens 10 to 14 . (9). w.

FloerkeA. Calyx 3.leaved, corol 3-petalled: style 2-cleft: capsule bladder-like, 2-grained. 15. 22-froserfinacoides, (false-mermaid) peduncles long, solitary. (-) $\mathrm{g}-\mathrm{y}$.

B. Flowers with a spathe or glume, without a flerianth.

Amaryllis. Corol superior, 6.petalled, unequal : filaments unequal in proportion or direction, declined, inserted in the throat of the tube. 9. 17atamasco, (atamask lily) corol liliaceous, stamens declined. 24. $\mathrm{r}$.

Hypoxis. Glume-like spathe 2-ralved: corol superior, 6-parted, permanent : capsule elongated, narrow at the base: seed roundish. 10.17-erecta, (star grass) scape 2 to 4 -flowered. 2!.y.

Allium. Spathe many-flowered: petals inferior, ovate, spreading, sessile. (Flowers in close umbels or heads.) 9. 16-horrum, (leek).umbelliferous, leaves flat. 24.-ascalonicum, (eschallots) umbelliferous, leaves awlform. 2\% e - -iricoccum, (garlic) umbelliferous, scape half-terete. 24.-cefia, (onion) scape bulging, longer than the terete leaves. 4. e.-schoenoprasium, (chives) scape and leaves terete, nearly equal. 2!.e.-sativum, (common gallick) bulb-bearing, leaves flat. 21. e.-canadensis, (wiid union) bulb-bearing, leaves linear. 21.

Galanthus. Petals 3, concave, superior : nectaries 3, small, emarginate: stigma simple. 6. 17nivalis, (snow-drop) leaves linear, keeled radical; flowers single. 24 . w.

Narcissus. Petals 6, equal, superior: nectary bell-.orm, 1-leafed, including the stamens. 9.17- 
jonquilla, (jonqui!) pelais thrice as long as nectary. 21. e.-hiseudo-narcissus, (daffodil) pelals equalling nectaries. 2f.e.

Pontederia. Corol inferior, 6-cleft, 2-lipped: capsule fleshy, 3-celled, many-seeded: three stamens commonly inserted on the tip, and three on the tube, of the corol. 6. 17-cordata, (pickerel weed) leaves heart-oblong. $4 . \mathrm{b}$.

C. Flowers without spathe, heriantls or glume.

Agave. Corol erect, superior, tubular, or funnel-form : filaments erect, longer than the corol: capsule triangular, many-seeded. 10.15-virsinca, (agave) stemless, leaves serrate, scape simple $4^{\circ}$ $\mathrm{g}-\mathrm{y}$.

Aletris. Corol superior, funnel-form, wrinkled, somewhat 6-cleft; with the stamens inserted on the base of its divisions : capsule 3-celled, many-seeded, half-inferior when mature. 10.16-aurea, (false aloe) tlowers spiked, nearly sessile, co. rols rough, leaves lance-sword-form. $2 \frac{4}{\mathrm{y}} \mathrm{y}$-w.

Hearerocallis. Corol 6.parted, tubular-funnel-form : stamens deciined : stigma small, simple. 10, 17-fava, (day lily) segments of corol acute. $\boldsymbol{4}$. y-e.-fulva, 3 inner segments of corol obtuse $24 \cdot y=\mathrm{e}$.

Dracakna. Corol inferior, 6-parted; or 6-petalled cohering at the base: filaments somewhat thickened in the middle: berry with 3 one-seeded cells. 11. 12-borealis, (dragoness plant) nearly stemless, leaves oval. 24. w.

Conostylus. Corol aore than half-inferior, 6cleft, permanerit, with branching hairs : anthers erect : style conic, 3-parted : stigma simple: capsule opening at top, 3-celled, many-sceded.-americana, corol woolly within, scage corymb-panicled, leaves grass-sword-form. Discovered in New-Jersey by Fursh. 
Erythronidm. Corol liliaceous, inferior, 6-petalled; petals reflexed, having 2 tubercle-form nectaries at the base of the three inner alternate petals. 11. 14-dens-canis, (dog-tooth violet, or adder-tongue) scape naked, leaves 2, glabrous spotted. 2\%. $y$.

Asparagus. Corol superior, 6-parted, erect ; the three inner petals reflexed at the apex : berry 3-celled; many-seeded. 11.12-officinalis, (asparagus) leaves fascicled, bristle-form. 27 . y. e.

Hyacinthus. Corol roundish or bell-form, equal 6-cleft : 3 nectariferous pores at the top of the germ: stamens inserted in the middle of the corol: cells somewhat 2-seeded. 10. 16-botryoides, (grape hyacinth) corol globular, leaves channelled. 21.b.-orientalis, (garden hyacinth) corol funnel-form, leaves keeled. $2 \%$.

Lilium. Corol inferior liliaceous 6-petalled; petals with a longie udinal line from the middle to the base : capsule with the valves connected by hairs crossing as in a sieve. 10.14-fhiladelthicum, (red lily) flowers about 2 or 3, erect; leaves mostly whorled. $r-y-$ canadense, (yellow lily) flowers pendant, leaves whorled, y.-suferbum, (superb lily) leaves whorled below; scattered above; flowers pendant revolute. $y$.

Tulipa. Corol 6-petalled, liliaceous : style none. 10.14-suaveolens, (tulip) petals obtuse, stem hairy. e. $\rightarrow$ gesneriana, petals obtuse, stem glabrous. e.

Convallaria, Corol inferior 6.cleft : berry 3. celled, spotted before ripening. 11.12-racemosa, (clustered solomon-seal) corol wheel-form, raceme terminal, leaves alternate sub-sessile. $\boldsymbol{\psi}$.w. - - mul. tiflora, peduncles axillary, leaves alternate somewhat clasping. 21. w.-bifolia, tetrandrous; corol wheel-form, 4. cleft, leaves 2 heart-ovate. 21 . w.

Uvularia. Corol inferior, 6-petalled, with a 
nectariferous hollow at the base of each petal : filaments very short. 11. 14-pierfoliata, (bellwort) leaves perfoliate. $2 ! \cdot y \cdot-$ sessilifolia, leaves sessile, lance-ovate. 4 . $y$.

Orontum. Receptacle spadix-like, cylindric, covered with florets : spathe 0 : corol 6 petalled, naked: style 0 : follicle 1-seeded. 2. 7-aquati. cum, (floating arum) leaves lance-ovate, glabrous, tapering base. $2 \%$.

Narthecium. Corol 6-petalled, inferior, spreading, permanent : filaments thread-form, hairy: capsule prismatic: seed appendaged at both ends. 10. 16-americanum, (false asphodel) having a clasping: bract. 2. y.

Ornithogalum. Corol 6-petalled, erect, permanent, spreading above the middle: filaments dilated or subulate at the base : capsule roundish, angled, 3-celled : seed roundish, naked. 10 16umbellatum, (star of Bethleheni) peduncles longer than bracts, corymbs. few-flowered. 21 .w.

Acorus. Receptacle spadix-like, cylindric, covered with florets: corol 6-petalled) naked : corol 0 : capsule 3-celled. 2.7-calamus, (sweet flag) spilse projecting from the side of a sword-form leaf. 24.

\section{Flowers without corol.}

Juscus. Glume 2-valved: calyx 6-leaved, permanent: stigmas 1 or 3-celled, 3-valved, manyseeded. 5. 13-tenuis, (rush grass) leaves chanel. led, panicle cymed. 4.-sylraticus, leaves flat, hairy, panicle cymed. 24-camfiestris, leaves flat, hairy, spikelets terminal. 2\%. (Very small.)-efusus, (bull-rush) culm round, smooth, panicle lateral. 2 .

* Trientalis, Polygonum, Laurus, Corydalis.

Order. II. Digynia.

Oryza. Calyx, glume 2-valved, 1-flowered : 
corol 2-valved, adhering to the seed. 4. 10-sativa, (rice) culm jointed, leaves clasping, panicle terminal (:). e.

* Uimus.

Order III. Trigyia.

Medeola. Calyx o : corol deeply 6-parted, revolute : berry 3 -seeded. 11.12-virginica, (Indian cucumber) leaves in distarit whorls. 4. w.

Melanthium. Polygamous. Calyx o: corol wheel-form, 6-parted or 6-petalled, each part with 2 glands at the base : stamens from the lengthened claws of the petals : capsule ovatish, somewhat 3 . cleft at the apex, s-celled: seeds membrane-winged. numerous. 10. 13-virginicum, (black-flower) flowers panicled, petals oval. 4. y.

Veratruas. Polygamous. Calyx o: corol 6petalled, without glands: capsules 3, many-seeded. (The staminate flowers have the rudiment of a pistil.) 10.13-viride, (itchweed, or swar p heilebore) panicle clecompound, leaves rerved. $\boldsymbol{\psi}$. g.-luteum, (white hellebore) raceme simple. 4 . w.

Helonias. Calyx o: corol 6-petalled : styles distinct: capsules 3.celled, 3-horned, few-seeded. 10. 13-latifolia. (helonias) leaves lance-awlform, scape nearly leafless, spike short. $\boldsymbol{4}$. p.

Scheuchzeria. Calyx 6-parted inferior : corol o: anthers linear: stigmas sessile, lateral: capsules 3, inflated, 1 or 2 -seeded. (Sometimes the calyx is taken for the corol.) 5. 13-qualustris, (less flowering-rush) capsules 3,5 , or 6 , stigmas lateral.

Zigadenus. Calyx 6-leaved, spreading, with 2 glands above the narrow base of each leaf : siamens inserted in contact with the germ: capsules membranaceous, 3-celled, many-seeded. (vome。 times the calyx is taken for the corol.) - slaberri- 
mus. (zigadene) leaves lance-linear, narrow, Iong. 2\%. b

Trilliva Calyx 3-leaved, inferior, spreading : corol 3-petalled : berry 3-celled, many-seeded. 11. 12--cernuum, (wake-robin) flowers peduncled, pendant. p-w.-rhomboideum, flowers peduncled, crect or erectish; Val. erectum, album, and grandi. florum p. or w.

Triglochin. Calyx 3-leared, inferior: corol 3-pctalled, resembling the calyx: stigmas sometimes 6: capsules deliscent at the base. 5. 13maritimum, (arrow-grass) capsule 6-celled, grooved.

Rumex. Calyx 3-leaved: petals 3, converging; stigma many-cleft : seed 1 , naked, three-sided. 12. 28-crisfia: (docls) valves ovate entire, all bearing grains. 4.- blutusifolia, valves toothed, mostly bearing frrins. 4.-aquatica, valves ovate entirc, grains obsolete. h.-britanica, valves entire, all bea:ins grains, leaves obsoletely sheathed. 4 .ciciocelli, (sorrel) leaves hastate. 2\%.

\section{Order XIII. Polyandria.}

Azrsia. Calyx 3-lcared : petals 3 : capsuleg nu:neruss, 1-secded. 5. 13-h!antugo, (water-plantair) leares heart-ovate. 2\%.w.

\section{CLASS VII. IIEPTANDRIA. Order I. Monogyata.}

Triestalis. Calyx 7-lcaved: corol 7-parted, eciual, flat: berry juiccless : number of stamens variable. 20. 3s-eurohece, (chick-wintergreen) learcs lanccolate, clustercel at the top. $2 \%$ w.

Esculus. Calyx inflited, 4 or 5-toothed: corol 4 or 5-petalled, inserted on the calyx, uncqual, pubescent: cajisule 3-celled : seeds large, chestnut-form. 23. 66-hiffucastanum, (horse-chestnut) leaves 7-fringed, corol 5-pecalled. $b_{6} \cdot c_{\text {. }}$ 
Order IV. Tetragyita.

Saurunus. Calyx an ament with 1-flowered scales: corol 0 : germs 4 : berries 4,1 -seeded. 2 . 6-cernuus, (lizard-tail) scales of the ament petioled, many-spiked. 4 .

* Polygonum orientale.

\section{CLASS VIII. OCTANDRIA.}

\section{Order I. Monogyia.}

\section{A. Flowers sufherior.}

Oxycocces. Calyx superior 4-cleft : corol 4parted, the divisions nearly linear, revolute : filaments converging, anthers tubular, 2-parted : berry many-seeded. 18. 51-vuigaris, (cranberry) stem creeping, leaves acutish, revolute. $h$. r-w. - macrocarhus, stems rising, leaves obtuse, flat. $h$.

Gaura. Calyx 4-cleft, tubular : corol 4-petalled, ascending towards the upper side : nut 1 -seeded. 17. 88-biennis, (virginian loosestrife) leaves lanceolate, toothed. $\delta$. $r$.

Epilobium. Calyx 4-cleft, tubular: corol 4petalled: capsule oblong and of great length : seeds feathered. 17. 88-fialustre, (willow-herb) stem teretes, leaves lanceolate toothletted. 24.r. - tetragonum, stem grooved-4-sided, leaves serrulate. 2\% r.-augustifolium, leaves lance-linear, entire, scattered. 4. p-b.

Oenothera. Calyx 4-cleft, tubular, caducous, divisions deflected : petals 4 , inserted on the calyx: stigma 4-cleft: capsule 4-celled, 4-valved : seeds not feathered. 17.88-biennis, (scabish, or treeprimrose) leaves lance-ovate, stamens shorter than corol. Large. \$. y.-humila, leaves entire, obtuse, capsule obovate. Small. 4. y.

B. Flowers inferior.

RHExis, Calyx pitcher-form, 4 or 5 -cleft : pe$\mathrm{E}$ 
tals 4 , oblique, inserted on the calyx : anthers declined : capsule 4-celled, within the calyx, setose : seeds numerous, cochleate. 17. 90-virginica, (deer-grass) leaves 3-nerved, ciliate-serrate. $24 \cdot \mathrm{p}$.

AcEr. Polygamous. Calyx 5-cleft : corol 4 or 5-petalled: samaras 2, united at the base, 1-secded. 23. 66-saccharinum, (sugar-maple) leaves acuminate-toothed, 5-lobed-palmate, sinuate, base sub-cordate. h.y.-rubrum, (soft-maple) leaves sub-5-lobed, gash-toothed, unequally. $h . r$.

Jeffersonia. Calyx 5-leaved, coloured, caducous : corol 8-petalled : cap̄sule obovate, sub-peduncled, 1-celled, dehiscent below : seeds many, oblong, arilled at the base-difhylla (twin-leaf) stemless, peduncle naked, 1 -flowered. 2\%. w.

Dirca. Calyx o: corol tubular, limb obsolete, unequal : stamens longer than the tube of the corol : berry, or drupe, 1. seeded. (The corol may be taken for a coloured calyx.) 31. 25-palustris, (leatherwood) leaves oval, flowers axillary, 2 or 3 in a hairy bud-like involucre, $h$. w.

Tropoeolum. Calyx 4 or 5-cleft, coloured, spurred : petals 4 or 5 , unequal : nuts leathery, sulcate. 23. 73-majus, (nasturtion) leaves peltate. 24. y.e.

* Chrysosplenium varies into the $2 \mathrm{~d}$ order of this class.

\section{Order III. TRIGYNiA.}

Cardiospermum. Calyx 4-leaved: petals 4:

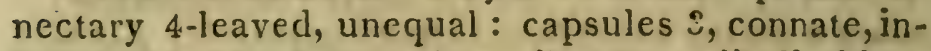
flated. 23. 65-halicacabum, (heart-seed) climbing, leafets gash-lobed. . w.

Poiygonum. Calyx inferior, 5-parted, coloured: corol o: seed 1, angular, covered with the calyx. (Stamens and pistils vary in number-The calyx in some species might be taken for a corol.) 12. 28-aviculare, (knotgrass). flowers axillary, 
stem procumbent. (). w.-hydrofifier, (water-pepper) stamens 6 , styles 2 ; spike filiform, nodding, leaves spotless. (․)w.p.-ficrsicaria, (persicaria) stamens 6 , styles 2, peduncles smooth, stipules ciliate. (). w.p.-hensylvanicum, styles 2 , peduncles bristly, stipules not ciliate. (-) p.-scandens, climbing, leaves cordate. 21.W.-convolvulus, twining, leaves heart-arrowform. $)$. w-arifolium, stem prickly, leaves hastate. $2 \% \cdot r$ - - virginicum, stamens about 5, styles 2, coloured calyx 4-cleft, leaves broad-ovate ciliate. $\boldsymbol{\psi}$. w. maritimum, flowers axillary, stern erect. w.-sagitalum, stem decumbent, prickly, leaves arrow-form. - . w.p.- -orientale, stamens 7, styles 2, leaves broad-ovate, stipules hairy. Very tall. (- r, e-fagohyrum, (buckwheat) stem smooth, branching. e.

\section{CLASS IX. ENNEANDRIA.}

\section{Order I. Monogynia.}

Launus. Calyx 4 to 6-parted : corol o: nectaries 3 , two-bristled glands, surrounding the germ: drupe 1 -secded. (Stamens vary from 3 to 14-often diœcious-The calyx may be taken for a corol.) 12. 27-sassafras, (sassafras) leaves 3-lobed and simple. h.y-benzoin, (spice-bush) leaves simple, acute both ends. $h \cdot y \cdot$

\section{Order ili. Trigfinia.}

RHeum. Calyx none : corol 6-cleft, permanent ; seed 1, 3-sided. 12. 2s-tralmatum, (rhubarb) leaves palmate roughish. e.

\section{CIAASS $\mathrm{X}$. DECANDRIA.}

Order I. Monogyxia.

A. Flowers many-hetalled, irregular.

Podalyria. Calyx 5-cleft, somewhat 2-lipped; corol papilionaceous, wings of the length of the 
banner: legume inflated, smooth, many-seeded. 3i.93-tinctoria, (wild indigo) stipules obsolete, leaves wedge-form. 24. y.-australis, stipules twice as long as petiole. $\psi$. b.

Cercrs. Calyx 5-toothed, gibbous below: corol papilionaceous, banner under the wing, short: legume oblong. 33. 93-canadensis, (judas-tree) leaves corciate. $h$.r.

CAssia. Calyx 5-leaved: corol 5-petalled : anthers, 3 lower ones beaked : legume membranaceous. 33.93-chamaecrista, (cassia) pinnate leafets many-paired. (-). y.-marilandica, pinnate leafets 8-paired. 24.y.

RHodora. Calyx 5-toothed : corol 3-petalled: stamens declined: capsule 5-celled. 18.50-canadensis, (rhodora) umbelled, leaves oval entire. h. r.

\section{B. Flowers 5-petalled, regular.}

Ciethra. Calyx 5-parted, permanent: style permanent: stigma 3-cleft: capsule 3-celled, 3valved, surrounded by the calyx. (Spiked) 18. 51 -alnifolia, (sweet pepper-bush) leaves obovate, serrate. $h$.w.

Pyrol a. Calyx 5 parted: anthers with 2 pores: capsule 5-celled, dehiscent at the angles. 18.51umbellata, (bitter wintergreen) peduncles umbelcorymbed, leaves lance-obovate. $\boldsymbol{\psi}$.w.-macula$t a$, peduncles 2 -flowered, leaves lance-ovate, whitish near the midrib. 4. w.-secunda, raceme many-flowered turned to one side, leaves ovate serrulate. 4. w.-rotundifolia, (shin-leaf) raceme many-flowered, leaves round. 4 . w.

Ruta. Calyx 5-parted : petals concave: receptacle surrounded by 10 nectariferous dots: capsule lobed. (Petals sometimes 4, and stamens 8.) 26. $81-$ sraveolens, (rue) leaves decompound of more, petals entire. 
Dronaea. Calyx 5-leaved : stigma fringed: capsule 1-celled, gibbous: seeds numerous. (Petals sometimes 6.) 14.73-muscifula, (Venus' flytrap) radical leaves with a terminal appendage resembling a rat-trap. $4 . \mathrm{w}$.

\section{Filowers 1-fnetalled.}

Andromeda. Calyx 5-partecl, inferior: corol ovate or roundish, with a 5 -cleft reflexed mouth : capsule 5-celled, with partitions contrary. (Stamens sometimes 8.) 18.51.- haniculata, (whitebush) corols roundish, panicled, leaves lance-obovate, stem whitish crack-barked. h. w.-calyculata, (leather-leaf) racemes one-sided, leafy, leaves lance-oblong, dotted. $h$. w.

RHododendron. Calyx 5-parted : corol somewhat funnel-form and oblique : stamens declining: capsule 5-celled, 8.50-maximum (rosebay) coroal wheel-form, umbel dease terminal : leaves paler beneath. $h \cdot r$.

KaLmia. Calyx 5.parted; corol wheel-salverform, with 10 horns beneath and 10 cavities within: capsule 5-celled. 18.50-latifolia, (broad-leaf laurel) corymbs terminal, leaves leathery ovate. oval. h. w-r.-angustifolia, (narrow-leaf laurel) corymbs lateral, plant small. h.r.

Vaccinium. Calyx 5-toothed or 5-parted: corol bell or pitcher-form, 5-cleft, the divisions reflected: filaments inserted on the germ with the corol : berry 4 or 5-celled, many-seeded. (The foreign species are sometimes octandrous.) 18.51 -corymbosum, (whortleberry) corymbs bracted, corol sub-cylindric, leaves pubescent beneath. $h$. w-p.-resinosum, racemes bracted, corol ovate, leaves sprinkled with resinous dots. $h \cdot \mathrm{p}$. - tenellum, raceme sessile, leaves smoothish, serrulate, oboval. $\zeta . w$. 
Epigana. Calyx double, outer 3-leaved, inner 5-parted: corol salver-form; capsule 5-celled, many-seeded. 18.51-refens, (trailing arbutus) leaves heart-ovate, stem creeping. $h . w-r$.

Gualtheria. Calyx inferior, double, outer 2leaved, inner 5-cleft: corol ovate : capsule 5-celled, invested with the inner, berried calyx : nectary 10-pointed. 18. 51-firocumbens (spicy wintergreen) leaves obovate. $2 \% \cdot w$.

Arbutus. Calyx inferior, 5-parted : corol ovate, pellucid at the base : berry 5-celled. 18. 51 -uva-ursi, (bear-berry) leaves wedge-obovate, stem procumbent. $h$. $\mathrm{r}$.

LEDUM. Calyx 5-cleft: corol flat, 5-parted: capsule 5.celled, dehiscent at the base. (Stamens sometimes 5.) 18.50-latifolium, (labrador-tea) leaves oblong, downy beneath, stamens about $5 . h$. w.-buxifolium, leaves ovate, glabrous, corymbs terminal. $h_{2} . w$.

\section{Calyx none.}

Monotropa. Corol 10-petalled: 5 outer with nectariferous hollows at the base : capsules 5 -valved. (A fifth part of the carpogenation sometimes wanting.)-lanuginosa, (beech-drops) flowers spiked, woolly. w. Whole plant yellowish white.-uniflora, (bird's nest) stem l-flowered. w. Whole plant ivory white.

* Geranium, Rhexia, Pertulacca, Lythrum. Orier II. Digynia.

HYDRANGEA. Calyx 5-toothed, superior: colol 5-petalled : capsule 2-celled, 2-beaked, dehiscent wetween the beaks. 13.85-arborescens, (hydrangea) stamens 8 to 10 , leaves oblong-ovate, toothed. h. w. Vid. Hortensia.

SAXIFRAGA. Calyx 5-parted: corol 5-petalled: capsule 1-celled, 2-beaked; many-seeded. 13. 84 -i'ernalis, (early saxifrage) leaves oval crenate. 
4. w. - hensylvanica, leaves lance-oblong, toothed. 2\%. w.

Trarela A. Calyx 5-parted: corol inserted on the calyx ; 5-petalled, petals entire: capsule 1celled: 2-valved, 1 valve larger. 13. 84-cordifolia, (mitre-wort) leaves cordate, petals longer than the calyx. H. w.

Mitella. Calyx 5-cleft: petals 5 on the calyx pinnatifid: capsule 1 -celled, 2 -valved, valves equal. 13. 84-difinylla, (false sanicle) leaves cordate, scape-like stem 2-leaved. 2 . w.

Chrysosplenium. Calyx 4 or 5-cleft, half-superior, coloured: corol o: capsule 2-beaked; ]celled, many-seeded. (Lateral flowers 8-stamencd.) 13 86-ophositifulium, (golden saxifrage) leaves opposite, heart-form, crenate. $\boldsymbol{\psi}$.

Dranthus. Calyx inferior, cylindrical, 1-leafed, with 4 to 8 scales at the base: petals 5 with claws: capsule cylindrical, 1-celled, dehiscent at the top. 22. 82-caryofhyllus, (pink) petals crenate-lobed, scales of calyx ovate acute. 24. r. - pllumarius, petals many-cleft, scales of calyx ovatish. 24. r-w.barbatus, (sweet-william) flowers aggregate, scales of the caly $\mathrm{x}$ as long as the tube. $\boldsymbol{\psi}$.

Saponaria. Calyx inferior; l-leafed, tubular, without scales: petals 5 , with claws: capsule oblong, 1-celled. 22.82-officinalis, (soap-wort) caly $x$ cylindric, leaves lance-oval. (-).r.-vaccaria, caly $\mathrm{x}$ conic, 5 -angled, leaves ovate sessile. $($.$) .$

Scierantuus. Calyx inferior, 1-leafed: corol 0 : seeds 1 or 2 inclosed in the caly $x$ : stamens inserted on the calyx. 2\%. 86-annuus, (knawell) stem spreading, caly $\mathrm{x}$ becoming sharp-toothed. $($. s.

\section{Order III. Trigyita.}

Arenarta. Calyx inferior, spreading, 5-leavcd: petals 5, entire: capsule 1.celled, many-seed- 
ed. 22. 82-lateriflora, (sandwort) stem simple, corol longer than calyx. 4. w.-serhyllifolia, stem branched, corol shorter than calyx. 3. W.-siric$t a$, leaves linear-subulate, calyx striate, $4 \cdot y \cdot-$ canadensis, stem prostrate glabrous, stamens 5, capsule globose. $\mathrm{O}$.

Stellaria. Calyx 5-leaved, inferior, sprearing: petals 5, deeply cleft, mostly to the claw, spreading : capsule ovate, 1-celled, many-seeded, 6-toothed at the top. 22. 82-fiubera, (starwort) petals longer than calyx, leaves sessile ovate ciliate. 4. w.-alsine, petals shorter than calyx, stem decumbent.. w.

Silene. Calyx 1-leafed, inferior, conic: petals 5, with claws appendaged at the mouth: capsule imperfectly 3-celled. 22. 82-jiensylvanica, (catchfly) viscid-pubescent, root-leaves wedge-form, stem-leaves lanceolate. w-r.-virginica, panicle forked, calyx downy and cylindric when in flower. 24. w-p.-antirrhina, peduncles 3.cleft, petals emarginate, leaves lanceolate.. .

Cucuralus. Calyx inferior, l-leafed, bellform, inflated : petals 5 with claws, not appendaged at the mouth: capsule 3 -celled. 22.82-stellatus, (bladder-campion) leaves in fours, petals manycleft. 2\%.w.-behen, stem forked, calyx bladderlike. 24. w.

Hortensia. Flowers deformed : florets solitary : calyx 5-toothed, minute : corol 5-petalled. The gay flowers composing the cyme, have a large coloured, permanent petal-like 5-leaved calyx, and a minute caducous 4 or 5 -petalled corol: stamens 8,10 or 11 . 13.85-sheciosa, (changeable hydrangea) leaves broad-ovate, tapering to both ends, serrate, cymed. $\mathrm{g}-\mathrm{r}$. This is the plant so common in flower pots, improperly called hyderindia. 


\section{Order V. Pentagyia.}

Sedus. Calyx inferior 5 -cleft: petals $5: 5$ nectariferous scales at the base of the germ : capsules 5. 13.83-telehthium, (liveforever, or orpine) leaves tooth-serrate, stem erect, corymb leafy. 24 . e.-hortulaccoides, creeping, leaves round-spatulate. h.w-e.-anacamfiseros, (stonecrop) decumbent, leaves wedge-form. e.

Penthorum. Calyx 5-10-cleft : petals 5 or 0 : capsule 5-cuspidate, 5-celled. 13. 83-sedoides, (Virginia orpine) stem angular, leaves serrate, alternate. 2f. y.

Oxalis. Calyx 5-leaved inferior : petals 5, cohering by the claws : capsule 5 -celled, 5 -cornered, dehiscent at the corners: stamens, with 5 shorter: outer ones adhering at their bases. 14.73-violacea, (wood sorrel) stemless, scape 2-cleft, umbelled. $24 \cdot \mathrm{p}$.-acetosella, stemless, scape 1.flowered, root granular. 24, w-p.-dillenii, stem erect, peduncles as long as leaves. O. y.-stricta, stem erect, peduncles shorter than the leaves. y.-corniculata, stem decumbent, rooting. (-). $y$.

Spergula. Calyx 5-leaved inferior: petals 5, entire: capsule ovate 1-celled, 5-valved. (Stamens often 5.) 22. 82-arvensts, (corn-spurry) leaves in whorls, peduncles reflexed in maturity, seed kidney-form. (-). w.-saginoides, leaves opposite, linear, smooth, awnless, stem decumbent, flowers pentandrous. Persoon asks whether this is not a variety of sagina trocumbens. Professor Ives considers all the American species of stergula and sagina, as a natural assemblage of plants, which ought to be united in one genus.

Agrostemma. Calyx 1 -leaved coriaceous: petals 5 with claws, border obtuse, entire : capsule 1 celled, many-seeded. 22. 82-githago, (cockle) bristly, petals entire. (-).r.-coronaria, (rose-cockle) downy, petals emarginate. $\hat{\delta}, r$. 
Cerastium. Calyx 5-leaved: petals 5, 2-eleft: capsule 1.celled, dehiscent at top, tooth-like. 22. 32-vulgatum, (mouse-ear chickweed) petals equalling the calyx, plant hirsute. w.-viscosum, viscous, petals hardly longer than calyx. w. - semide. candrum, pentandrous, petals shorter than calyx. w.

Lychnss. Calyx 1-leaved, oblong, smooth : petals 5, with claws: the limb somewhat 2-cleft: capsule 1 or 5-celled. 22.82-chalcedonica, (campion) flowers in fastigiate heads. 24. e.

\section{* Phytolacca.}

\section{Order X. Dechginia.}

Phytolacca. Calyx o: corol 5.petalled, calyx-like, inferior : berry 10-celled, 10-seeded. 54. 89-decandra, (pokeweed) leaves ovate acute, racemes simple: $2 \%$. W: berries red.

\section{CLASS XI. DODECANDRIA. \\ Onder. I. Monogyni.}

Asarum. Calyx 3 or 4-cleft, superior: corol o: stigma 6-cleft: capsule coriaceous, crowned with the calyx. 11.23-canadense, (wild ginger, or white snake-root) calyx reflexed, leaves in pairs, heart-reniform. 4 . p. root aromatic.-virginicum, leaves solitary, glabrous, cordate. 4 .

Hudsonia. Calyx tubular, 3-leaved, inferior: corol 5-petalled : stamens 15 : capsule 1 -celled, 3 valved, 3-seeded. 13.51-ericoides, (false-heatli) leaves.subulate-needle-form, hirsute, peduncles solitary, filiform. h. y.

Portulacca. Calyx 2.cleft, inferior : corol 5petalled: capsule 1 -celled, opening transversely : columella 5, filiform. 13. 86-oleracea, (purslane) leaves wedge-form, succulent, flowers sessile. $y$.

Lyturum. Calyx 6, 8, 10 or 12 -toothed, inferior: petals 5 or 6 on the calyx: capsule 2-celled, 
many-seeded, covered. 17.91-verticillatum, (milkwillowherb) flowers whorled, leaves opposite, generally in threes, lanceolate. $\%$. p.

Decumaria. Calyx 10-leaved, superior : corol 10-petalled: capsule about 8 or 10-celled, seeds numerous. 19.89-sarmentosa, (decumary) stem sarmentose, lower leaves round, upper ones lanceovate. h.w.

TALINUM. Calyx inferior, 2 or 5 -leaved : corol 5-petalled : capsule 1.celled, 3-valved, many seeded, seeds arillate: columella globose-teretifolium, (taliny) leaves cylindric, flesh ycorymbs terminal. 24. p.

\section{Order II. Digynia.}

Agrimovis. Calyx 5-toothed, invested with an outer one : petals 5 : seeds 2 , in the bottom of the calyx. 35. 92-eufatoria (agrimony) leaves inter: ruptedly pinnate. $4 . \mathrm{y}$.

* Delphinium.

\section{Order IIt. Trigyia.}

Euphorbia. Calyx 1-leaved, inflated : petala 4 or 5 , stancing on the calyx : capsule 3 -grained. 38. 96-maculata, (spotted spurge) stem prostrate, forked, leaves hairy spotted. (-). w. -helioscofia, (sun spurge) umbel 5 -rayed, then 3-rayed, forked, leaves wedge form. - polysonifolia, (knot -spurge) leaves entire, stem decumbent. 24.

RESEDA. Calyx 1-leafed, 4 to 6-parted: petals in many divisions: capsule 1-celled, dehiscent at the top: seed reniform (stamens 11 to 15 : styles 3, 5, or none) 54.64-odorata, (mignonette) leaves entire and 3-lobed, calyx long as petals, styles often. 24. y. e.-luteola, (dyers' weed) leaves lanceo. late, entire, flat, calyx 4.cleft. Found growing wild near New-Hayen, by professor Ives. 


\section{Order XIII. Polygynia.}

Sempervivum. Calyx 9 to $12 \cdot$ parted : petals 8 to 12 : capsules 12, many-seeded. (Stamens 16 of 20.) 13.83-tectorum, (house leek) leaves ciliate, offsets spreading. 4.-arboreum, branched woody. h.

\section{CLASS XII. ICOSANDRIA:}

\section{Order I. Monogynia.}

Philadelphus. Calyx 4-5-parted, superior: corol 5-petalled : style 4-cleft : capsule 4-5-celled, manyaseeded; seed arilled. 19. 89-coronarius (false syringa, or mock orange) leaves slightly toothed. $h$. w.

Prunus. Calyx 5-cleft, inferior : corol 5-petalled: nut of the drupe smooth with prominent seams at the sutures. 36. 92-borealis, fruit ovatish, umbels sub-corymbed, sessile, few-flowered, leaves acuminate, petioles with 2 glands. $h$.w.-virginiana, (wild cherry) flowers in erect compact racemes, leaves doubly-toothed, petioles with about 4 glands. h. w.- serotina (choak-cherry) racemes lax, serratures simple, lower ones sub-glandular. $h$. wcerasus (common cherry) umbels sub-sessile, leaves lance-ovate. $h$.w. e.-domestica, (common plum) peduncies sub-solitary, leaves lance-ovate. e.

Cactus. Calyx superior, 1-leafed, imbricate: petals numerous, in many series, the inner ones larger; stigma many-cleft: berry 1-celled, manyseeded, navelled. 13. 85-ofuntia, (prickly pear). nearly stemless, leaves very thick, succulent and proliferous. $y$.

MYrtus. Calyx superior, 5-cleft: petals 5 : berry 2 or 3-celled, many-seeded. 19.89-communis, (myrtle) flowers solitary. $h$. w.

AMYGDalus. Calyx 5-cleft, inferior : petals 5 : drupe with 2 nut perforated with pores : flowers 
sessile. 36.92-hersica, (peach) leaves serrate, flowers solitary, sessile. h.r. e. A variety, nectarine, has glabrous fruit.

* Lythrum.

EROM ORDER II. DYGINIA, TO ORDER Y. PENTAGXNIA.

Crataegus. Calyx superior, 5 -cleft : petals 5 : styles generally 3 or 5 : berry mealy: seeds 2 to 5 , bony. 36. 92.-coccinea, (thorn bush) leaves heartovate, petioles and calyx glandular. h.w. $t a$, leaves heart-ovate, petioles and calyx without glands. h.w.-crus-galli, leaves wedge-obovate, styles 2. h. W-oxyacantha, (quick-set) leavcs obtuse, 3 -cleft, styles 3.

SoRBUs. Calyx 5-cleft, superior: petals 5 : styles 2 to 3 : berry pomaceous : seeds 2 or 3 cartilaginous. 36. 92-americuna, (mountain ash, or service tree) corymbed, leaves pinnate, leafets serrate. $h$.

Aronia. Calyx 5-toothed : petals 5: fruit pomaceous : berry 5 or 10 -celled; cells 1 or 2 -seeded; seeds cartilaginous. 36.92-botryafizum, (shadflower) leaves oblong-oval cuspidate, flowers race. med, petals linear. h. w.-arbutifolia, (choak-berry) corymbed ; leaves obovate crenate-serrate, midrib glandular above. $h$. w-ovalis, leaves roundoval, flowers racemed, petals obovate, ciliate. 々. These species were formerly arranged under the genus hyrus.

Mespinus. Calyx 5-cleft, superior, divisions generally foliaceous : corol 5 -petalled : styles 2 5 : drupe or berry with from 2 to 5 bony seeds.36. 92-hyracantha, (medlar, or evergreen thorn) thorny, leaves lance-ovate, crenate, calyx obtuse. $h$. -sermanica, (common medlar) without thorns, h. 


\section{Order V. Pentagynia.}

Prrus. Calyx 5-cleft, superior : corol 5-petalled: pome 5-celled, many-seeded. 36.92-communis, (pear) leaves ovate, flowers corymbed. . $_{\text {. w. e. }}$ -malus, (apple) leaves ovate, flowers in sessile umbels. h.r-w. e.-cydonia, (quince) leaves entire, flowers solitary. h.w. e.-coronaria, (crab-tree) leaves cordate, angular, flowers corymbed. $h_{2} \cdot \mathrm{r}$-w.

Spiraea. Calyx 5-cleft, inferior, spreading: corol 5-petalled : capsule 2-valved within, manyseeded. 36. 92-alba, (steeple bush, or hand-hack) leaves wedge-lanceolate, serrate, glabrous, panicled. $h$. w.-tomentosa, leaves lanceolate, downy, racemes compound. h. p.-trifoliuta, (Bowman's root, or Indian physic) leaves ternate, flowers sub. panicled. 24. w.

Mesembryanthenum. Calyx superior, 5-cleft : petals numerous, linear, cohering at the base : capsule fleshy, many-seeded, turbinate. 13.87-crystallinum, (ice-plant) very branching, leaves ovate with ice-like papillæ. w. e.

Order XiII. Polygynia.

RosA. Calyx urn-form, 5-cleft, fleshy, contract. ed towards the top: petals 5 : seeds numerous, bristly, fixed to the sides of the calyx within. 35.92. - caroliniana, (swamp-rose) germ globular hispid, peduncles somewhat corymbed and hispid, stem with stipular prickles. h. r.-rubiginosa, (sweet-brier) germs ovate, peduncle and germ hispid, petioles and stems with recurved prickles. $h$. r.-lucida, (wild rose) germ globular-depressed, somewhat bristly, leaves oblong-oval, glabrous, flowers mostly in pairs, $h . r$ - harvifiora, germ globular-depressed, bristly, prickles on the stipule strait, leaves oval, flowers in pairs. $h . r$.

Rubus. Calyx 5-cleft, corol 5-petalled: pistils 
numerous: berry composed of many juices, 1 -seeded, acines. 35.92-occidentalis; (black-raspberry) leafets 3 , downy beneath, stem prickly, petioles cylindric. h. w.-odoratus, (flowering raspberry) leaves simple, palmate. $h$. r.-hrocumbens, (creeping blackberry) procumbent, peduncles and petioles hook-prickly. $h$. w.-strigosus, (red-raspberry) without prickles, strongly bristled, leafets in threes or fives, pinnate, white down beneath. $h$.w. -villosus, (high blackberry) leafets 5 or 3, ovalpointed, sharp-serrate, downy both sides, stem and petioles prickly. h.w.-saxatilis, (brier herb) stem herbaceous, creeping, leafets 3 , nearly glabrous. 21. w.-ideus, (garden raspberry) leafets 5 and 3 , stem prickly, petioles channelled. h. w-e.

Dalibarda. Calyx 5-cleft: corol 5-petalled: styles long, caduc eous, 5 to 8 ; berry composed of dry granulations. 35.92-rehens, (dalibard) leaves cordate, stoloniferous. 24.w.-fragarioides, leaves ternate, crenate-lobed, ciliate. 2\%. y.

Fragaria. Calyx 10-cleft, 5 alternate divisions smaller : corol 5-petalled: receptacle ovate, berry-like, caduccous. 35.92-virginiana, (American strawberry) calyx spread, down on petioles erect, on peduncles close. 2\%. w.-vesca, (garden strawberry) calyx reflexed, down on petioles much spread. 2\%.w.

Potentilla. Calyx 10-cleft, 5 alternate divisions smaller: corol 5-petalled: seeds awnless, roundish, rugose, fixed to a dry small receptacle. 35.92- humila, (five-finger) nearly stemless, hairy, erect, peduncles 1 -flowerd shorter than petioles. 21.y.-reftans, stem creeping, leafets obovate serrate, peduncles 1.flowered. 21.y.- simfllex, stem erect, simple, hairy, leaves oblong-oval, peduncles lateral, 1-flowered, long. 24.y-canadensis, stem ascending, rough-haired. 2!, y-norwegica, leaves 
ternate, stem forked. 24. y.-argentea, (silver cinquefoil) leafets in 5 wedge-form divisions, silvery whitish, prostrate. 2 . y.

GEum. Calyx 10-cleft, 5 alternate divisions smaller: corol 5.petalled : seeds with a bent awn: receptacle columnar, villous. 35. 92-rivale, (avens, or herb bennet) flowers drooping, awns bent in the middle, root-leaves ly rate. 24. p.-virginianum, flower's erect, awns twisted near the end, stemleaves ternate. $24 \cdot y \cdot w$.

Comanum. Calyx 10-cleft, 5-alternate divisions smaller : petals 5 , smaller than the calyx : receptacle ovate, spongy, permanent, villous. 35. $92-$ halustre, (marsh fivefinger) leafets in threes, fives, or sevens, serrate. 2 . p.

Calycanthus. Calyx 1-leafed, pitcher-shaped, scurfy, with coloured divisions: corol none: styles many, with glandular stigmas : seeds many, tailed, in the succulent calyx. 35. 92-floridus, (Carolina allspice) branches spreading, leaves broad-oval. $h$. p. e.

\section{CLASS XIII. POLYANDRIA.}

Order I. Monogynia.

Chelidonium. Calyx 2-leaved: corol 4-petalled: silique-like capsule 1-celled, linear : seeds crested, many. 27.62-majus, (celandine) leaves pinnate-lobed, umbels axillary. $24 \cdot y$.

ACTAEA. Calyx 4-leaved, caduceous : corol 4petalled : berry 1-celled : seeds half orbicular : 26 . 61-sficata, (neclace weed, or bane-berry) racemes terminal. 24. w.

AserRum. Calyx 4-leaved : 2 interior leafets, cordate, large : corol 4-petalled: filaments dispos. ed in 4 parcels : capsule oblong, 1-celled, 2 valved, included in the caly $x$ leaves. 20. 68-crux.andreae, (St. Andrew's cross, or St. Peter's wort) 
stem terete, leaves ovate, panicle dichotomous. h. y.-stans, stem ancipital, leaves oblong, flowcrs vary into the $3 \mathrm{~d}$ order. $y$.

Cistus. Calyx 5-leaved, 2 of them smaller, cor. rol 5-petalled: capsule 3-valved, opening at the top. 20.80-canadensis, (rock-rose) stamens prostrate on the petals, leaves alternate, lanceolate. 24 . y. Late in autumn this plant sends off curved ice crystals from near the root, of a very singular structure.

Sarracenia. Calyx double, 3 and 5-leaved: corol 5-petalled: stigma peltate, covering the stamens: capsule 5-celled. 13.22-nurfurea, (side. saddle flower) leaves cup-form with bordered mouths, radical. 2. p. Found in abundance in the lakes on the Catskill mountains near Benton's turnpike.

Citrus. Calyx 5-cleft : petals 5, oblong: fila. ments dilated at the base, and several parcels : berry 9 to 18 celled-Polyadelphous. 18.70-aurantium, (orange) leaves oval with winged petioles. h.w. e.-medica, (lemon) leaves ovate with linear petioles. 2.. w-e. A variety limon (lemon) has the fruit roundish and small.

Papaver. Calyx 2-leaved, carlucous : corol 4petalled : cápsule 1-celled, dehiscent by pores under the permanent stigma. 27. 62-somniferum, (poppy) leaves clasping. (- p. e.-rheas, leaves pinnatifid. $)$. $r$.

Tilia. Calyx 5-parted : corol 5-petalled: capsule 5-celled, globular, coriaceous, dehiscent at the base, one-seeded. 37. 79-hubescens; (bass. wood) leaves truncate at the base and oblique, somewhat downy. $h$. wi-americana, leaves cordate, glabrous. $h . w$.

Sangeinaria. Calyx caducous, 2-leaved: corol about 8-petalled: capsule pod-like, ovate, 1F 2 
celled. 27. 62-canadensis, (blood-root) scape 1. flowered, leaves lobed. 24. w.

Podophyllum. Calyx 3.leaved : corol about 9petalled: berry i-celled, crowned with the stigma. 27. 61-heltatum, (wild mandrake) leaves peltate, pedate. 24. w.

Capparis. Calyx 4-leaved, coriaceous, caducous: petals 4 : stamens long : berry coriaceous, peduncled, l-celled, globular or pod-like-stinosa, (caper-bush) peduncles solitary, 1-flowered, leaves oval, alternate. w-r. e.

Nymphas. Calyx 4 to 7-leaved, larger than the petals : corol many-petalled : stigma marked with radiated lines : berry many-celled, many-seeded. 13. 22-odorata, (pond-lily) leaves round-cordate, leaves of calyx about as long as the petals. 21. w. -advena, leaves cordate, petals wedgeform, much shorter than the calyx. 4 . $y$.

Argemone. Calyx 3-leaved: corol 6-petalled: capsule semi-valved. 27. 62-mexicana, (horned poppy) leaves cut-pinnatifid, thorny. $\odot . y .-v a r i e-$ ty, alba, has white flowers.

From Order II. Digynia-to Order V. PenTAGYNIA.

Fothergillia. Calyx truncate, entire : corol o: filaments very long, clavate : germ bifid: capsule 2-celled or 2-horned: seed bony, solitary. 50 . 99-alnifolia, (Fothergill's bush) leaves wedgeform, spikes terminal. $h$.

PaEonia. Calyx 5-leaved: petals 5 : style 0 ; stigmas 2 or 3 : capsules pod-like, many-seeded. 26. 61-officinalis, (peony, erroneously called pina) leaves decompound. 24. $r$. In gardens the stamens usually change to petals.

Hypericum. Calyx 5-parted, divisions sub-ovate : corol 5-petalled: filaments often united at the 
base in 3 or 5 sets : styles 2 to 5 : capsules roundish with a number of cells equal to the number of styles. 20.68-herforatum, (St. John's wort) stem 2 -edged, leaves obtuse having pellucid dots. 21. y-harviflorum, corymbs terminal, forked, leaves sessile. 24. y.-virginicum, flowers 9-sta-

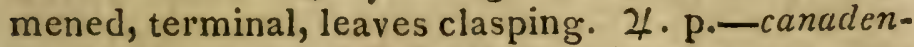
se, flowers peduncled axillary solitary, leaves sessile, stem square. $y$.

Delphinium. Calyx 0: corol 5-petalled, unequal: nectary 2 -cleft, horned behind : capsules 1 or 3, pod-like. 26. 61-stathisagria, (larkspur) leaves palmate obtuse-lobed, nectary 4-leaved. b. e.-azureum, leaves linear-many-cleft, spiked, spur curved. 2\%. p. e.

Aconitum. Calyx 0 : petals 5, upper one vaulted : nectaries 2 , hooded, peduncled, recurved: capsules 3 or 5, pod-like. 26. 61-nafiellus, (monk's hood) leaves 5-parted with 3-parted divisions. 24. p. e.

Aquilegia. Calyx 0 : petals 5 : nectaries 5 alternating with the petals and ending in horns beneath : capsules 5, distinct. 26. 61-canadlensis, (columbine) nectary horns nearly strait. 24.r-y.vulgaris, nectary horns incurved. 24. b-p.

Cimicifuga. Calyx 4-leaved: petals (or nectaries) 4, pitcher-form, cartilaginous : capsules 4: seeds scaly. (Petals, pistils and caly $\mathrm{x}$-leaves, variable.) 26. 62-americana, (bug-bane) flowers spiked, leaves decompound, pistils 5 or $6 . \%$. w.

Nigelda. Calyx none: petals 5 : nectaries 5 , three-cleft, within the corol : capsules 5, convex, 26. 61-damascena, (fennel flower) involucre 5-leaved, longer than the flower. (-). b.

* Reseda, Ascyrum, Caltha, Helleborus, Anemone, Hepatica. 


\section{Order XIII. Polygyia.}

Hydrastis. Calyx 0 : corol 3-petalled : berry composed of 1 -seeded acines. 26.61-canadensis, (orange-root) leaves binate, palmate, petioled. 2 . w.

Clematis. Calyx $0:$ petals, $3,4,5$, or $6:$ seed 8 compressed: styles fermanent, becoming long tails. (Some species are diœcious.) 26. 61-virginana, (virgin's bower) diœcious, climbing, leaves ternate with climbing petioles. 4 . W.

Thalictrum. Calyx $0:$ petals 4 or 5 : fila. ments very long : seeds without tails, striate, terete. (Some species are dicecious.) 26.61 -dioicum, (meadow rue) dicecious, leaves heart-roundish with obtuse lobes, peduncles axillary, shorter than leaves. 4. r. w.-cornuti, diœcious, leafets 3.cleft, panicles terminal. 2\%. w.-hoiygamum, hairy and slender, leaves more than decompound, panicle terminal: 24 . $w$.

Helleborus. Calyx 0 : petals 5 or many : neciaries tubular, 2-lipped : capsules 5 or 6, erectish, compressed, many-seeded. 26. 61-trifoliatus, (gold thread) scape l-flowered, leaves ternate with soundish crenate leafets. 4: w. Root creeping and golden yellow.

Caltua. Calyx 0 : petals 5 to 9 : capsules numerous, many seeded : nectaries 0 : (styles variable in number.) 26. 61-halustris, (American cowslip) leaves heart-reniform, crenate. $4 \cdot y$.

Averone. Calyx 0: petals 5 to 9 : seeds numerous, naked. 26. 61-nemorosa, (wincl-flower) stem simple, 1-flowered, 3-leaved with gashed leafets. 24. r-w.-thalictroides, (rue-anemone) stem simple, stem-leaves 6 whorled petioled, root-leaves long-peduncled. 24. w.-virginiana, stem forked, many-flowered, petals acute. $2 \cdot \mathrm{g} \cdot \mathrm{y}$. 
Hydropeltis. Calyx 6-leaved somewhat converging : corol o : capsules oblong, somewhat fleshy, numerous, 2-celled, 1 or 2-seeded. 26. 61hurhurea, (water-shield) leaves oval, peltate, floating. 2\%. $\mathrm{p}$.

Hepatica Calyx 3-leaved : petals 6 to 9 : seeds naked. 26. 61-triloba, (liverleaf) calyx-leaves broad-ovate obtuse, leaves 3-lobed obtuse. 21 . w-r. Var._acuta, calyx-leaves acute, leaves 3 to 5-lobed acute. Perhaps this variety ought to constitute a new species. The specimens found by professor Dewey, near Williams' College, are much firmer in their texture than the common kind; and differ materially in their general habit.

Annona. Calyx 3-leaved: corol 6-petalled: stigma obtuse: berries 1-celled, many seeded, roundish with a scaly bark. 52.76-glabra, (custard apple) leaves lance-ovate, fruit conic glabrous. e.

Liriodendron. Calyx 3.leaved: corol 6 or 9petalled, liliaceous : seeds in a samara, imbricate on a strobile-like spike. 52. 75-iulinifera, (tulip tree, or white wood) leaves 3-lobed, with the midd!e lobe abrupt. 乙. . w.

Magnolis. Calyx 3-leaved : corol 6 to 9-petalled : capsules numerous, imbricate on a strobile-like spike, 2 valved : seeds berried, pendulous. 52.75-glauca, (magnolia, or beaver tree) leaves oval obtuse glaucous beneath, petals obovate. 24 .w.

Nelumbium. Calyx 4 or 5 -leaved: petals 5 or more : nuts half immersed in a truncate receptacle, and crowned with the permanent style, 26. 61-luteum, (nelumba) leaves peltate, pecluncles and petioles glabrous. 4 . $y$.

Ranunculus. Calyx 5-leaved: petals 5, with claws and a nectariferous pore or scale on the inside of each : seeds numerous. 26.61-abortivus, (crowfoot) radical leaves cordate crenate, stem-leaves 
subternate. 2f.y.-acris, (butter-cup, or crowfooi) lower leaves 3 -parted many-cleft, upper ones linear. 4. y.-sceleratus, (water crowfoot) lower leaves palmate, upper ones digitate. 24.y.-hensylvanicus, calyx reflexed, leaves ternate 3 -cleft hairy beneath. ○. y.-fuviatilis, leaves all under water, capillary. $y-w .-f a s c i c u l a r i s$, leaves ternate-pinnate, root fascicled. 24. y-bulbosus, calyx reflexed, peduncles grooved, root bulbous. 4 . y.-refens, scions creeping, leaves linear entire. $4 . y$.

Trollius. Calyx 0: petals from 5 to 14 : capsules many, ovate, many-seeded. 26.61-americanus, (globeflower) petals about 5 obtuse, nectary shorter than the stamens. 2 . $y$.

Atragene. Calyx 2 or 4 -leaved, sometimes involucred: petals numerous, outer ones largest: seeds tailed. 26.61-americana, (atragene) leaves in fours, leafets cordate. Flowers large. $24 . \mathrm{p}$.

Adonis. Calyx 4-5-leaved: petals 5 or more, without nectariferous pores: seeds awnless. 26.61 -autumnalis, (pheasant's eye) fruit sub-cylindric, petals 5 to 8 . e.

\section{CLASS XIV. DIDYNAMIA. \\ Order I. Gymospermia.}

A. Calyxes 5-farted with the divisions or teeth necrly equal.

TExcrium. Corol deep-cleft on the upper side and without an upper lip; lower lip 3.cleft, the middle division rounded : stamens and pistils incurved, stamens exse't. 42.39-canadense, woodsage, or wild germander) whorls 6-leaved, leaves lance-ovate, serrate, spike crowded. 21.r.-virginicum, bracts shorter than peduncle, leares ovate. 24. $r$.

Isanthus. Calyx bell-form : corol 5-parted, 
tube strait, narrow; divisions ovate equal : sta. mens nearly equal: stigma linear, recurved. 42. 39-coeruleus, (blue gentian) viscid-hairy: leaves lance-ovate, tapering to both ends.

VERBENA. Corol funnel-form with a curved tube, border 5-cleft nearly equal : calyx 5-cleft : seeds 2 or 4 : sometimes but 2 stamens or 2 barren ones-urticifolia, (vervain) spikes filiform, leaves undivided ovate serrate. $\%$ - w-p.-hastata, leaves hastate below and lanceolate above. 2 . w-b. angustifolia, spikes filiform, leaves narrow lancelinear. Small. 4.r.-aubletia, spikes lax, leaves 3-cleft, erect. (-). r.-sturia, stems many, leaves many-cleft jagged. 24.w-1.-1innatifida, leaves pinnatifid.

Lavaxdula. Calyx ovate subdentate, bracts under-studded: corol resupinate : stamens in the tube. 42. 39-sticata, (lavander) Icaves sessile lance-linear, spike leafy.

Lamivar. Corol with the upper lip entire, vaulted; under lip 2-Jobed; throat with a tooth at each margin. 42. 39-amfllexicaule, (dead nettle) floral leaves sessile clasping gashed. (-). p.

Brachystemux. Corol with the upper lip sub-emarginate, lower lip obtusely 3-lobed: filaments very short nearly equal: seed oblong-cylindric. 42. 39-virginicum, (Virginian thyme) leaves lanceolate, flower's in small heads fastigiate, stem square. $2 \%$. w.

Pycnanthemum. Involucre bract-like, manyleaved, under small heads of flowers : calyx tubular, striate : corol with the upper lip sub-entire, lower lip 3-cleft : stamens nearly equal. 42.39aristatum, (wild basil, or mountain mint) bracts linear acuminate, leaves lance-oval with short petioles acuminate, plant downy. 2\% w-p.-incanum, bracts bristle-form, leaves oval petioled acuminate, 
downy. 2\%.w-p. Both species have a pleasant pungent taste.

Nepeta. Calyx dry: striated: corol with a longish tube, under lip with the middle division crenate, throat with a reflected margin: stamens approximate. 42. 39-cataria (catmint) flowers whorled, leaves cordate tooth-serrate. 4 . w-b.

Hyssopus. Corol with the under lip 3-parted, its intermediate division sub-crenate: stamens strait, distant. 42. 39-officinalis (hyssop) leaves lanceolate obtuse entire. 2\%. b. e.-nenetoides, leaves sub-cordate acute-toothed. $24 \cdot \mathrm{y}$.

Mextra. Corol nearly equal, 4-cleft : broadest division emarryinate: stamens erect, distant. 42.39 -viridis, (spear mint) spikes interrupted, leaves ses. sile, bracts bristle-form. 24. p.-nifierita, (peppermint) spikes obtuse, leaves petioled, ovatish. $\boldsymbol{\Psi}$. b. - borealis, (Canada mint) leaves petioled projecting. $24 \cdot \mathrm{b}-\mathrm{w}$.

Stachys. Corol with the upper lip vaulted, the lower lip reflexed at the sides, the middle division largest, emarginate : stamens reflexed towards the sides after discharging the pollen. 42.39 -asizera (false horehound) stem erect, with hairs pointing backwarcl, leaves lance-cordate, spike whorled. 2f. r.

Satureja. Calyx tubular, striate : corol with divisions nearly equal: stamens distant. 42.39.hortensis, (summer savory) peduncles axillary 1 or 3-flowered, leaves lance-ovate. (-). b. e.

Leonurus. Calyx 5-angled, 5-toothed, corol with the upper lip villose, flat, entire; lower lip 3-parted, middle division undivided. 42. 39-cardiaca, (motherwort) upper leaves 3-lobed, entire. 24. w.

MARRUBRIUM. Calyx salver-form, rigid, marked with 10 lines: corol with the upper lip 2-cleft, 
linear, strait. 42. 39-vulgare, (horehound) calys with 10 -hooked teeth, leares toothed. 4 . w.-sufiin un, (nodding horebound) leaves round-cordate, crenate, rugose-veined, teeth of the calyx 5 , strait. 4. $b \cdot p$.

Cuxina. Calyx cylindric, 5-toothed, throat villose, corol ringent ; upper lip erect, flat emarginate $: 2$ of the stamens usually barren. 42.39.-12uleginoides, (penny-royal) leaves oblong 2.touthed, flowers whorled.

Grechoma. Calyx 5-cleft : each pair of anthers approaching so as to exhibit the form of a cross. 42.39-hederacea, (ground ivy) leaves kidney-form crenate, stem square procumbent. $\psi$. b.

\section{B. Calyxes 2-linned.}

Melissa. Calyx dry, flattish above, with the upper lip sub-fastigiate: corol with the upper lip somewhat vaulted, 2-cleft; lower lip with the middie lobe cordate. 42.39-officinalis, (balm) whorls half-round, bracts pedicelled. 2\%. w.-nekeza, (low calamint) branching hairy, leaves ovate obtuse. $\%$. $\mathrm{w} \cdot \mathrm{r}$.

Trichostema. Corol with the upper lip falcate: the under lip 3-parted with the middle division small, oblong : filaments very long-exsert. 42. 39-dichotoma, (blue-curls)) stem forked. (-). b.

Diacocephalum. Corol with the throat inflated; the upper lip concave, the middle division of the lower lip roundish, notched. 42.40-denticulatum, (dragon head) flowers spiked, remote, leaves lance-obovate. 4. p.-virginianum, flowers spiked, crowded, leaves lance-linear. 4 . p.-variegatum, spikes 4-cornered short, leaves oblong. p.

Scutellaria. Calyx with an entire mouth, which is closed with a lid after the corol falls out: tube of corol bent. 42.39-lateriflora, (skull-cap) 
branching, leaves smooth with a rough keel, racemes lateral and leafy. 4 . b. Said to be highly efficacious in the hydrophobia.-Galericulata, leaves heart-lanceolate, crenate, wrinkled, flowers axillary. 24. b.

Origanum. Calyxes collected into a 4 -sided strobile-like cone, with broad intervening bracts: corol with the upper lip erect, flat: under lip 3 . parted, divisions nearly equal. 42. 39-marjorana, (marjoram) spike ternate, leaves oval obtuse. p. w. e-vulgare, spikes panicled, bracts longer than calyx. p.

Thymus. Calyx with the throat closed with hairs: corol with the upper lip flat, emarginate; lower lip longer. 42.39-vulgaris, (thyme) flowers in whorled spikes, leaves ovate revolute. p.e.serfiyllus, (mother of thyme) flowers in heads, stem decumbent, leaves flat. p. e.

Chinipodium. Involucre many-bristled, bractlike, under the whorl: perianth 2-lipped: corol with the upper lip flat, obcordate, strait. 42.39vulgare, (field thyme) stem simple, leaves above hairy remotely toothed. 4 . p.

Prunella. Calyx with the upper lip dilated: filaments 2-forked with an anther on one of the points : stigma 2-cleft. 42.39-hensylvanica, (selfheal, or heal-all) leaves lance-ovate toothed, upper lip of calyx 3-awned. 4 . p. A variety, ovata, has ovate leaves, and lanceolata, lanceolate leaves and blue flowers.

Pнry under lip of calyx shorter, 2-toothed.) 40.39-leftostachya, (phryma) leaves ovate serrate, spike slender terminal $\%$. $p$. 


\section{Order II. Angiospermia.}

A. Calyxes 2-cleft, or 2-leaved.

Obolaria. Corol 4-cleft, bell-form: capsule 1-celled, 2-valved, many-seeded: stamens from the divisions of the corol. (Persoon says the caly $x$ is 5 cleft-Muhlenberg says it is 2-leaved.) 40. 35virginica, (penny-wort) spikelets terminal, leaves opposite coloured. 4. r-w.

\section{B. Calyxes 4-cleft.}

Orobaxche. Calyx of 2-lobed lateral leafets : corol ringent: capsule 1-celled, 2-valved, manyseeded: glands under the base of the germ. 40.35 uniflora, (cancer-root, broom-rape) stem 1-flowered. b-w.-virginiana, stem branched. p.

Bartsia. Calyx lobed, emarginate, coloured: corol less than calyx ; upper lip longest : capsule 2-celled: seed angled. 40.35-coccinea, (painted cup) leaves cut-pinnatifid, bract 3 -cleft. $\delta . r . \$ \& y$.

Rhinantuus. Calyx inflated : corol ringent; the upper lip mostly compressed, the under lip spread 3-parted : capsule 2-celled, obtuse, compressed. 40. 35-crista.galli, (yellow-cockscomb) calyx glabrous, upper lip of corol 2-toothed at the apex, stem spotted. (.) y-p.-virginicus, corol with spreading throat, leaves sinuate-toothed. $y$.

EUPHRASIA. Calyx cylindric: corol 2-lipped; upper lip 2-cleft, lower lip 3-lobed, with the divisions 2 -cleft : lower anthers lobed spinose. 40. 35-officinalis, (eye-bright) lower lip emarginate, leaves ovate toothed.

Melamprrum. Corol, with the upper lip compressed, the margin folded back : capsule 2-celled, oblique, dehiscent on one side: seeds 2, gibbous. 40. 35-lineare, (cow-wheat) leaves linear-lanceo- 
late, the upper ones toothed, bristled at the base, flowers axillary. (-) $y$-w.

Schwalbea. Calyx tubular-inflated; upper division small, lower division large emarginate : corol ringent: capsule ovate, compressed, 2-celled, 2-valved with a double partition: seeds many, chaffy. 40.40-americana, (chaff-seed) sterı square, hairy, leaves lanceolate. $4 . \mathrm{p}$.

\section{Leaves 5-cleft or 5-leaved.}

ZAPANiA. Flowers capitate : calyx 5-tnothed: corol 5-cleft : stigma head-peltate, oblique : pericarp bladder-like, vanishing, connecting the two seeds. 40. 40-lanceolata, (fog-fruit) leaves lancelinear sharp-serrate, stem crecping:-nodiflora, jeaves wedge-form toothed, stem creeping.

Scrophularia. Calyx 5-clcft : corol sub-globose resupinate, middle divison of lower lip reflexed: capsule 2.celled. 40.40-marylandica, (figwort) leaves cordate serrate, stem blunt angled. 2 . p.-glabrata, leaves doubly-serrate. 24. p.

Eninus. Calyx 5-leaved: corol with the limb equally 5-cleft, lobes emarginate; upper lip very short, refiexed: capsule 2.celled. 40. 35-africanus, (erinus) flowers lateral sessile, leaves lanceolate, slightly toothed. p.

BIGxosia. Calyx 5-cleft cup-form: corol with the throat bell-form, 5 -cleft, ventricose beneath : capsule silique-like, 2-celled: seed membranewinged. 40. 45-radicans, (trumpet-flower) stem rooting, leaves pinnate. $h . r$.

Ruxlira. Calyx 5-parted, often 2 bracted : corol somewhat bell-form, limb 5-lobed : stamens conjugate : capsule tapering to both ends, toothed, clastically deliscent : seeds few. 40.36-strefiens, (ruel) leaves ovate cutirc, peduncles 2-flowered shor'to $24 \cdot \mathrm{p}$. 
Brchnera. Calyx obsoletely 5-toothed : corol with the limb in 5 equal divisions, the lobes cordate: capsule 2-celled. 40. 34-americana, (buchnera) spikes remotely flowered, leaves lanceolate 3-nerved. $24 . \mathrm{b}$.

Axtrrrhincm. Calyx 5-leaved or deeply 5parted, the two lower dirisions remote : corol ringent, spurred or with a prominent base, the gape closed with a prominent palate : capsule ovate, 2valved, dehiscent at the apex. 40,40-linaria, (snap-dragon) flowers in terminal spikes, leaves crowded together lance-linear. 2\%. y.-canadense, (toad-flax) flowers racemed, leaves alternate remote. (.). p.

Gerardia. Calyx 5-cleft : corol 2-lipped; lower lip 3.parted with emarginate lobes, middle one 2-parted: capsule 3.celled, dehiscent at the base. 40. 40-fava, (faise foxglove) hairy, leaves lanceolate gash-pinnatifid below, flowers axillary opposite. 21.5.-quercifolia, (oak-leaf foxglove) glabrous, stem panicle-like, pinnatificl leaves with lanceolate divisions, upper leaves entire. 2\%. y.-hurfurea, (dwarf foxglove) leaves linear, very branching. (-). p.-fiedicularia, (lousewort foxglove) hairy, leaves pinnatifid doubly-serrate. $44 \cdot \mathrm{y}$.

Digitalis. Calyx 5-parted: corol bell-form, ventricose, 5 -cleft: capsule ovate, 2 -celled. (Flowers racemed.) 40. 40-hurhurea, (foxglove) corol obtuse with an entire upper lip, ieaves hairy. $\delta$.p.

Pedicularis. Calyx 5-cleft or obliquely truncate : corol ringent : capsule 2-celled, mucronate, ublique : seeds numerous, coated. (Leaves manycleft.) 40, 35-canadensis, (lousewort) calyx obliquely truncate, spike leafy short, upper lip of co. rol with 2 bristles. 21. y. \& p. - gladiata, (high heal-all) spikes leafy rough-hairecl, flowers alternate, $4 \cdot y-p$.

$$
\text { G } 2
$$


Mrruzus. Calyx prismatic, 5-toothed : corol ringent, upper lip folded back upon its sides : stigma thick : capsule 2-celled, many-seeded. 40.45 -ringens, (monkey-flower) leaves sessile lanceolate glabrous. $2 \%$ b.

Chelone. Calyx 5-cleft or 5-leaved : corol ringent, inflated; the upper lip emarginate obtuse, under lip slightly s-cleft: the rudiment of a smooth filament between the two tallest stamens: capsule 2 -celled. 40.15-glabra, (snake-head) leaves lanceolate serrate, upper ones opposite. 2 . W.-obliqua, leaves all opposite. 4 . p.

Fentstemon. Calyx 5-cleft or 5-leaved: corol ringent inflated: the rudiment of a bearded filament between the two tallest stamens: capsule 2 celled. (Taken from the last genus.) 40. 35- $n 26-$ bescens, (beard-tongue) stem hairy, upper lip short. 4. $\mathrm{p}$.

Martynia. Calyx 5-cleft : corol ringent almost bell-form: capsule woody and barked, with a hooked beak; 4-celled, 2-valved. 40. 42-firoboscidea, (unicorn-plant) stem branched, leaves cordate, fruit hook-beaked. (O). W.

\section{CI.ASS XV. TETRADYNAMIA.}

Order t. Siliculosa.

All plants of this class have flowers with 4-leaved calyses and cruciform corols.

A. Silicle without a notch at the end.

Dra RA. Silicle oval-oblong, valves flattish, parallel to the partition: style none. 39.63 -verna, (whitlow-grass) leaves lance-oblong acutish subserrate, with short stiff hairs, scape naked, petals 2-cleft. O. w.-caroliniana, leaves ovate hirsute, scape naked simple smooth, silicle linear. 4 . w.

BunIAs. Silicle somewhat 4 -sided, not opening 
by valves, caducous; 2 or 4 -celled, wrinkled. 39. 68_edentula, (sea rocket) leaves obovate sinuate, silicle with 2 smooth one-seeded toothless joints. (). Bigelow.

B. Silicle with a notch at the end.

Alyssux. Filaments of the two shorter stamens marked with a tooth: silicle emarginate, often hairy. 39.63-sativum, (madwort) leaves clasping, flowers corymbed. (-). $\mathrm{y}$.

Cochlfaria. Silicle thick, rugose, many-seeded, 2 -valved; the valves gibbous obtuse. 39.63officinalis, (scurvy grass) root-leaves round-cordate petioled, stem-leaves sessile oblong. $\hat{\delta} \cdot 17$-armoracia, (horse-radish) root-leaves lanceolate, crenate. 2\%. w.e.

LEPIDIUs. Calyx spreading : corol regular: silicle emarginate, cordate, many-seeded : valves carinate, partition contrary. 39.63-sativum, (pepper-grass) leaves oblong, many-cleft, glabrous. 0 . w. e.-virginicum, leaves below pinnatifid, above serrate. 2f. W.

Thlaspr. Calyx spreading: silicle emarginate, obcordate, many-seeded; valves resemble two boats with the keels outward. 39.63-bursa-hastoris, (shepherd's-purse) silicle wedge-obcordate, root-leaves pinnatificl. 0 . w.-camplestre, (mithridate mustard) silicle roundish-cordate, leaves arrow-form. (-) w.

\section{Ordek II. Silliquosat}

A. Calyx leaves converging or closed ution the corols, when the flowers are mature.

Arabrs. Glands 4, one within each leafet of the caly $\mathrm{x}$, of the size of the reflected scale : silique

+ Plants of this order resemble each other so nearly, that long descriptions are necessary. 
compressed, torulose, subdivaricate. 39.63-that iana, (wall-cress) radical leaves oblong petioled, cauline leaves lanceolate sessile, stem erect roughhaired at the base, petals twice as long as the caly $\mathrm{x}$. (i). w.- tuberosa, stem simple, leaves obtuse lanceolate toothed, lower leaves sub-petioled, root tuberous-fibrous. 4 . w.-lyrata, leaves glabrous, radical leaves lyrate, stem leaves linear-lanceolate sub-falcate. 6. w. -hisfida, radical leaves liontooth-lyrate, stem-leaves lanceolate with forked hairs, stem branchy glabrous. $\mathbf{4}$. w.-canadensis, leaves lanceolate toothed glabrous, or with very soft hairs. w.

TuRritis. Calyx converging, erect : corol erect: silique very long, angled, striate. 39.63-laevigata, (tower-mustard) leaves glabrous, radical ones obovate serrate, cauline ones lance-linear entire clasping. $\delta$. w.-hirsuta, leaves all hispid, cauline ones clasping, silique 4-angled. 7 . w.

Dentaria. Calyx longitudinally converging; silique bursting elastically with revolute valves: the partition thick and fungus-like: stigma emarginate. (Roots always fleshy with tooth-like pro. cesses.)-difhylla, (tooth-root) stem with two ternate leaves nearly opposite and unequally gashed. 2f. w-y.-laciniata, leaves triternate, leafets 3-parted linear toothed, racemes terminal many-flowered. 2f. w-p:

Hesperis. Calyx closed, shorter than the claws of the petals : petals when bent ohliquely, are linea! or obovate: silique sub-terete : stigmas forked, with converging apexes. 39.63-finmatifida, (rocket) leaves sharply serrate, upper ones lanceolate, low. ers one pinnatifid, siliques shortish sub-peduncled, 4. w.-tristis, stem branched bristly spreading. y. e.-matronalis, (dame's violet) stem simple crect. leaves lance-ovate denticulate. $\boldsymbol{4}$. w. e.

Enximum. Calyx closed ; silique columnar, 
exactly 1-sided. 39.63-officinale, (hedge-mustard) leaves runcinate, siliques pressed close to the stem. 2. y-barbarea, (wintercress) lower leaves lyrate, with a roundish terminal lobe, upper leaves obovate toothed. 4 . $y$.

Brassica. Calyx erect, converging : partition extending beyond the valves of the silique : seed globose: glands betwe en the short stamens and pistil, and between the long stamens and calyx. 39. 63.-oleracea, (cabbage) leaves smooth glaucous repand-lobed. $\delta$. e.-nafıs, (rape) leaves smooth, upper ones lance-cordate clasping, lower ones lyrate toothed, silique torulose. S . e.-rana, (turnip) root orbicular depressed, radical leaves jyrate scabrous, cauline ones entire smootls. $\hat{\delta}$.e.

Raphanus. Calyx closed, setose : silique torose, somewhat jointed, terete, not opening by valves, I or 2-celled : glands between the short stamens and pistil, and between the long stamens and calyx. 39. 63-rafhanistrum, (white radish) leaves lyrate, silique 2 -celled when immature, and not jointed, in maturity 1 -celled and jointed. - .w. or y. e.-sativus, (garden radish) leaves lyrate, silique terete torose 2-celled. (-).e. Var. fusiformis, root spindle form ; globosa, root globose; niger, root blackish and sharp-tasted.

Cheiranthus. Calyx closed, two of the leafets gibbous at the base: petals dilated : silique when young with a glandular tooth each side : stigma 2-lobed : seed flat. 39.63-chiri, (stock- julyflower) leaves lanceolate acute glabrous. e.

B. Calyx-leaves stiread, not lying closed ution the corol when the flowers are mature.

Cardamixe. Calyx leaves spreading but little: stigma entire : a single gland between each of the stiort stamens and the calyx: silique long bursting 
elastically with revolute valves. 39.63-fiensylvani$c a$, (American watercress) branching, glabrous, leaves pinnate, leafets angle toothed roundish-oblong obtuse, siliques narrow erect. w.-virginica, leaves pinnate, leafets lanceolate 1 -toothed or eared at the base. (In habit much resembles the shepherd's purse.) $4 \cdot \mathrm{w}$ - - hirsuta, leaves without stipules, leafets roundish mostly repand-toothed petioled, flowers tetrandrous. w.

SISyMBRIUM. Caly $\mathrm{x}$ and corol spreading : silique bursting, with a short terete beak, valves straitish. 39. 63-nasturtium, (English watercress) silique declined, leaves pinnate with sub-cordate leafets. 24. r. Southern states.-amphibium (water-radish) silique declined oblong ovate, leaves pinnati. fid serrate, petals longer than caly $\mathrm{x}$. 24. w.

Sinapis. Calyx spreading: corol with strait claws: glands between the short stamens and pistil, and between the long stamens and calyx : partition extending beyond the valves of the silique, ensiform. 39. 63. nigra, (mustard) silique glabrous, leaves linear-lanceolate at top. (- y. e.-aiba, silique hispid, leaves pinnatifid. $e$.

\section{CLASS XVI. MONADELPHIA.}

Order III. Triandria.

Sistainchius. Spathe 2-leaved: perianth $0:$ corol superior, 6-cleft, tubular: style 1 : capsule 3celled. 6. 18-anceps, (blue-eyed grass) culm 2. edged, leaves ensiform, fruit globular glabrous. 24. b.

\section{Order V. Pentandria.}

Passiflora. Calyx 5-parted, coloured : corol 5-petalled, on the calyx: nectary, a triple filamentous crown within the petals : berry pedicelled. 34. 97 -coerulea, (passion flower) lcaves palmate 5- 
CLASS XVI. ORDERS X AND XIII. $7 \%$

lobed. b. South America_lutea, leaves cordate 3-lobed. y. Southern States.

Erodicar. Calyx 5-leaved: corol 5-petalled: nectariferous scales 5 -alternating with the filaments : arils 5,1 -seeded, awned, beaked at the base of the receptacle; awn spiral, bearded within. (Taken from the geranium.) 14.73.-cicutarium, (stork's bill) peduncles many-flowered, leaves pinnate with sessile gash-pinnatifid leafets, stem pros. trate rough-haired. 0. p.

* Lysimachia, Linum, Anagallis.

Order X. Decandria.

Geraniux. Calyx 5-leaved: corol 5-petalled, regular: nectariferous glands 5, adhering to the base of the long filaments : arils 5,1-seeded, awned, beaked at the head of the receptacle: awn naked, strait. 14.73-maculatum, (spotted cranebill, or crowfoot) stem dichotomous erect, leaves gash. 5-parted, peduncles 2-flowered. 24. p.-robertianum, (herb-robert) calyx 10-angled pilose, peduncle 2 -flowered, leaves in 3 or 5 three-cleft pinnatifid divisions. (-). p.-columbinum, peduncles thrice as long as the 5-parted leaves, capsules glabrous, (i). b.

Mrmosa: Calyx 5-toothed : corol 5-cleft, 5petalled, or 0 : stamens capillary, very long, 4 to 10. or more, sometimes not united: legume some. Limes jointed. 33. 93-brachyloba, (sensitive plant) unarmed, leaves doubly pinnate 8-paired. 24 . r. we.-glandulosa, unarmed, leaves doubly-pinnate 12-paired. 2f.w. e-sensativa, prickly. e.

Order xili. Polyandria.

Gordonia. Calyx simple, 5-leaved or 5-parted : corol 5-petalled adhering at the base : style 5-cornered, stigma 5-cleft : capsule 5-celled: co- 
Iumella columnar : seeds 2 with leaf-like wings. 37. 74-lasianthus, (leaf-seed) leaves leathery glabrous, flowers long-peduncled. $h . w$. Southern states. - franklinia, flowers sessile. ${ }^{2}$. w:

SidA. Calyx simple, angular, 5-cleft: style many-parted : capsules many, 1 or 3 -seeded. (Pedicel articulated under the apex.) 37. 74-sfinosa, (Indian-mallows) leaves lance ovate, toothed, stipules bristle-form. y.-abutilon, leaves heartroundish. (- y.-rhombifolia, leaves lance-wedgeform. $z \cdot y$.

Hrbrscus. Calyx double, outer one many-leaved, inner one about 5 -cleft : stigmas 5 : capsule 5 or i0-celled, many-seeded. 37. 74-moscheutos, (hibiscus, or marsh-mallow) leaves ovate acuminate serrate and downy beneath, petiole bearing the flower. 2!.w-p.-flalustris, (marsh hibiscus) leaves ovate sub-trilobed, flowers axillary. 2t. p.-syriccus, (althaea frutex, or syrian mallows) leaves wedge-ovate 3 -lobed toothed. 2 . p. Southern states.-esculentus, (okra) leaves cordate 5-lobed, outer caly 10 to 12 -parted, caducous. 2.w.e.

MALva. Calyx double, outer one 3.leaved, inner one 5.cleft: capsules many, 1-seeded. 37.74 . -rotundifolia, (low mallows) stem prostrate, leaves heart-reniform doubly-crenate or 5 to 7 crenatelobed. 4 . b.p.-caroliniana, stem prostrate, leaves 5-lobed cut-toothed. O.-sylvestris, (common mallows) stem erect, leaves with 7 sharpish lobes. 21 .b. -americana, leaves ovate rough. 3 . $y$.

Althaea. Calyx double, outer one 6 or 9. cleft : capsules many, 1-seeded. 37. 74.-officinalis, (hollyhock or marsh mallows) leaves oblong-ovate, toothed, 3-lobed, downy. 4. p-w. e.-rosea, (common holly-hock) leaves cordate crenate 5 to 7 angled rugose. $\hat{o} \cdot \mathrm{p} \cdot \mathrm{y} \cdot \mathrm{w}$. e.

Gossypium. Calyx double, outer one 3-cleft: 
CLASS XVII. ORDER VI TO VIIII. 7\% capsule 4-celled, seeds involved in wool. 87.74.herbaceum, (cotton) leaves 5-lobed mucronate. (). w. e.

\section{CLASS XVII. DIADELPHIA.}

From Order Vi, Hexandria, to Order Vitr, Octandria.

Fumaria. Calyx 2-leaved, caducous : corol ipregular, spurred at the base: filaments 2 , each with 3 anthers: capsule drupe-like, 1-celled, 1seeded, not opening by valves; seed affixed to the side of the cell. 24. 62.- officinalis, (fumitory) leaves more than decompound with leafets wedgelanceolate gashed, stem with spreading branches. \%. r.

Corydalis. Calyz 2-leaved: corol ringent 1 or 2-spurred : filaments 2, membranaceous, each with 3 anthers: capsule silique-like, many-seeded. 24. 62.-cucullaria, (colic weed) stemless, corol 2. spurred, scape naked, leaves more than decompound. 21. $y-w$. Those found near IVilliams' College are mostly hexandrous-they have also a twoleaved bract, so near the calyx in the immature state, as to give the appearance of a 4-leaved calyx.-rosea, 1-spurred, leaves pinnate-pinnatifid, silique one and a half longer than peduncle. $\boldsymbol{\psi}$.r.y.

Polygala. Calyx 5-leaved, unequal, 2 of the leafets wing-like, larger, coloured : corol irregular (or rather, calyx 3-leaved, corol imperfectly papilionaceous) capsule obcordate, 2-celled, 2 -valved. Keel of corol sometimes appendaged. 33. 35.KEEL APPENDAGED.-paucifolia, (evergreen snakeroot) keel crested, flowers terminal about in threes, leaves ovate near the flowers. 2). r.-rubella, (milkwort) keel crested, raceme nearly bractless, wings of corol 3-nerved obtuse longer than keel, stem-leares linear obtuse, radical ones ovate. $2 \% \cdot r$. 
-incarnata, keel crested, spiked, stem branched crect, leaves alternate subulate. r.-KEEL NoT APPENDAGED.-sanguinea, spikes in heads, peduncles scurfy, stem angled, erect, branched. r.-verticillata, leaves narrow-linear whorled, flowers with spaces between them.w.r.-senega, (seneca snakeroot) spike filiform terminal, stem simple, leaves lance-oblong. $4 . \mathrm{w}$.

\section{Order X. Decandria:}

A. Strmens united in one set.

Amorpha. Calyx bell-form 4 or 5-cleft : ban: ner ovate, concave, wings and keel 0 : legume 2seeded, falcate. 32.93.-fruticosa, (false indigo) somewhat shrubby, leaves glandular petioled, calyx 4.toothed. h. b-p.

Lupinus. Corol 2-lipped : anthers, 5 oblong and 5 roundish : legume coriaceous, torulose. 32 . 93-perennis (lupine) leaves divided, calyxes not appendaged alternate. 4 .b.-villosus, leaves simple, calyxes appendaged half-whorled. 2!.w-p.hilosus, leaves divided, calyxes appendaged whorled.

Crotolaria. Corol with the banner cordate, large : keel acuminate : filaments connate, with a dorsal fissure: style curved; legume pedicelled, turgid. 32. 93-sagittalis, (rattle-box) leaves lanceoblong, stipules acuminate decurrent, opposite to the racemes. (9). y.-harviflora, leaves lanceolate, upper stipules decurrent very short opposite to the racemes."

Genista. Calyx 2-lipped, 2 upper teeth very short: banner oblong, reflexed back from the pistil and stamens. 32.93-tinctoria, (dyer's kneedgrass) leaves lanceolate smooth, branches striate. e.

Arachis. Calyx 2-lipped: corol upside down: legume gibbous, torulose, veiny, coriaceous. 32.93 
hyprogaea, (pea-nut) stem procumbent, leaves pinnate abrupt. O. y.

B. Stamens in 2 sets; generally 9 in one set, and 1 alone in the other.

1. Legume many-seeded, stigma hubescent.

PIsuM. Calyx with the divisions leaf-like, about equal : banner protruding 2 folds : style compressed, carinate, villose above : legume without down at the suture. 32. 93-sativum, (pea) petioles cylindric, stipules rounded at the base and notched. 고. w. e. Var. umbellatum, bouquet pea, peduncles many-flowered- terminal : quadratum, garden pea, fruit cinereous : humile, dwarf pea, leafets bijugous.-maritimum, (sea pea) petioles flattish above, stem angular, stipules some what arrow-form. $2 \%$.w.

Lathyrus. Calyx with the two upper divisions shorter : style flat, villose above, broader towards the top. (Stem mostly winged, leafets 2 or more terminated by a divided tendril.) 32. 93-odoratus, (sweet pea) peduncles 2 -flowered, leafets ovate-oblong. (-) p. e.-venosus, stem not winged. (-).

VICIA. Calyx emarginate above, 2-toothed; 3 strait long teeth below : banner emarginate: stigma bearded transversely on the lower side. 32. 93.- americana, (wild vetch) peduncles many-flowered shorter than the leaves, stipules half-arrowform. 24. p.-husilla, pecluncles axillary 1 -flower. ed, leaves about in sixes, stipules half-arrow-form. y. w.-milchilla, peduncles many-flowered, leafets about 7 -paired retuse-mucronate, legume 2 -seeded. w. p. This species was found last summer by professor Mitchill of New-York, and sent to the Italian naturalist, C. S. Rafinesque; who, discovering it to be a new species, gave it this specific name.-sativa, (tare) legumes sessile axillary mostly in pairs, stipules toothed. b-w. -faba, (gar. 
den bean) legumes nearly sessile axillary 3 together swelling at the seeds, stipules sagittate. (-) e:

Phaseolus. Keel, stamens and style spirally twisted together. 32. 93-STEMS TWINING.-vulgaris, (common poled-bean) racemes solitary short$\mathrm{er}$ than the leaves, peduncles in pairs. $\mathrm{p}$. w. e: helvolus, (wild bean) flowers in heads, leafets 3 . cornered oblong. Q. p.-STEM ERECT.-nanus, (bush-bean) bracts larger than caly $x$, stem smooth. . w. p. e. All of this genus are usually called kidney-beans.

Dolichos. Banner with two oblong paralle processes at the base, compressing the wings beneath them. 32. 93-furhureus, (cowhage) stem twining glabrous, petioles hairy. - p.- phaseo. lus, stem erect, seeds globular, with coloured hilums. w.

Roвinis. Calyx 4-cleft, upper division 2-parted: banner large, reflexed, roundish : legume gib. bous, elongated. 32. 93-viscosa, (locust tree) racemes erect, branches and legumes glandular-viscid. h. r.-hishida, stem bristly without thorns. $h_{2}$. p. - $h$ seudo-acacia, (false-acacia) racemes pendant, legumes smooth, stipules spinous. h.w.

INDIGOFERA. Calyx spreading : keel with a subulate spur both sides: legume linear, small, terete or quadrangular. 32. 93-tinctoria, (indigo) leaves pinnate, racemes shorter than the leaves, stem somewhat woody. e.

2. Legume many-seeded, stigma without hubes. cence.

Vexillaria.* Banner very large, spreading; overshadowing the wings: calyx bell-form or tu-

* This name is given as a substitute for that of the 1712th genus of Persoon, which is so severely censured by Doctos Smith in Rees' Cyclopxdir. 
bular : legume linear, very long, acuminate. 32. 93-virginiana, (butterfly-weed) leaves ternate oblong pubescent, calyxes twinned. b.--mariana, leaves ternate, calyx cylindric, bracts lance-linear. w.p.

Galactia. Calyx 4-toothed, 2-bracted: petals all oblong, banner broader incumbent : stigma obtuse: legume terete : seed roundish. 32. 93.slabella (milk-way plant) glabrous, leaves oblongovate somewhat emarginate. 24 . p.

Grycine. Calyx 2-lipped, the tooth of the low. er lip longest : the end of the keel curling upwards, and, in appearance, pushing back the banner: style incurved with the keel : legume oblong compressed. 32. 93.- angulata, (wild bush-bean) leaves ternate, side ones 2-lobed, end one ovatish. w.p.monoica (pea-vine or wild liquorice) stem twining, hairy, leaves ternate ovate. 6 - b-w. afios (groundnut) pinnatifid leaves with 7 lance-ovate leafets, stem twining, root tuberous. 24. p.

GaLega. Calyx with subulate teeth nearly equal: legume torulose, teretish. (Very glabrous pinnate leaves.) 32. 93.-virginica (goat's rue) calyx woolly, leafets oval oblong. 24. p.

\section{Legumes ferw-sseded.}

MeDichao. The keel deflected from the direction of the banner: legume compressed, cochIeate. 32. 93. - sativa (lucerne clover) leafets oblong toothed, peduncles racemed. 24. b. e-lufiulina, procumbent, leafets obovate, legume reniform 1-seeded. 今. y - maculata, leafets obcordate toothed, stipules toothed. $y$.

Astragalus. Calyx 5-toothed: keel obtuse: legume somewhat 2-celled, incurved at the suture below. 32. 93._canadensis (milk vetch) spreading, leafets (about 21) glabrous, legume sub.cylindric.

$\mathrm{H} 2$ 
2. y-carolinianus, erect, leafets (about 41) hairy beneath, legumes swelled. 24. y.-sccundus, procumbent, legumes pointing one-way. 2\%. p.

\section{Legunes aboust 1-seeded.}

Thifolius. Flowers sub-capitate: legume in. cluded in the calyx, not opening by valves, 2 to 4 seeded. (Leaves always ternate.) 32. 93-12ra$t \in n s e$ (red clover) heads dense-ovate, 4 of the calyxteeth equal, stipules awned. 2\%.r.-rapens" (white elover) creeping, heads umbelled, leafets emarginate serrulate. 4. w- -arverse (field clover, or rabbit-foot) heads cylindric, calyx-teeth bristleform longer than corol, leafets narrow.oborate. r-w.

Melizotus. Flowers racemed : calyx tubular, 5-toothed: keel simple, shorter than the wings and banner: legume rugose, longer than the calyx. (Taken from the trifolium.) 32.93-offinalis (melilot clover) stem erect, stipules lance-subulate. w.

I,ESPEDEZA. Calyx 5-parted, divisions nearly equal : keel of the corol transversely obtuse : Jegume lens-form, unarmed, l-seeded. (Leaves always ternate. Taken from the hedysarum.) 32. 93-iafitala (bush-clover) erect, leaves sessile with oblong leafets, little heads in terminal heaps, legume much smaller than the calyx. p. A variety, angustifolia, leaves lance-linea!, peciuncles long. - holystachio, erect and rery downy, leafets round ovate, legume and calys equal. w.-frocumbens, frocumbent, hairy, leafets ovate. $\psi$. p.

5. Legumes jointed, or in loments.

Hedrsarum. Calyx 5.cleft : keel of corol transversely obtuse : loment many-jointed, joints 1seeded, compressed, generally hispid. (Most, or all, the species in the Northern States have ternate leares.) 32. 23-ernalense (bush-trefoil) leaves 
lance-oblong, stipules filiform, flowers racemed. 2\%. p. -violaceum, leaves oval obtuse, racemes umbelled, flowers in pairs. 2\%. p.-divergens, leaves oblong obtuse, racemes longer than petioles, flowers in pairs. 4. p.-nudiflorum, leaves roundish-ovate pointed, scape panicled glabrous. 24. p.-rotundifolium, stem prostrate hairy, leaves orbicular. 4.p. canescens, leaves roundish, stem angular ciliate bristly. 2\%. p.

Struosanthes. Calyx tubulär, very long, bearing the corol which originates higher than the top of the germ : loment two-jointed, hooked. (Sometimes the pod is in a single joint.) 32.93 -elatior (pencil-flower) leafets lanceolate, bracts ciliate, flowers spiked. $y$.

Aeschynomene. Calyx 2-lipped: loment compressed, one suture strait, another lobed; joints truncate, 1-seeded. 32.93-hisfida (false sensitive plant) petioles with bristly tubercles, leafets manypaired linear obtuse. $y-r$.

\section{POLYADELPHOUS PLANTS.}

The genera Ascyrum and Hypericum were placed in the class Rolyadelfinia by Linneus, and are still retained in that class by many authors. We, following Persoon's method, have removed them to the class holyandria, and left the class 110. byadelshia out of the system.

\section{CLASS XVIII. SYNGENESIA.}

Order I. Polygamia Equalis.

A. Florets ligulate.

TRAGOPOGON. Calyx simple, many-leaved : re. ceptacle naked: egret plumose and stiped. $\nmid 45.53$

$\dagger$ Pull off the corol, stamens and styles; whatever is left on the germ is the egret. In some flowers the stipe to stir 
-dandelion (goat's beard) stemless, leaves enisiform, flower with a scape. 2f.y.-forrifolium, having a stem. $\hat{\delta}$. p.

APARGIA. Caly $x$ imbricate : receptacle naked: egret plumose, sessile. (Taken from the leontodon.) 49. 53-autumnalis (false hawkweed) leaves lanceolate smoothish tooth-pinnatifid, scape branching.y. Dr. Bigelow supposes it came to Boston from Europe.

Leontodon. Calyx double : receptacle naked: egret plumose, stiped. 49.53-taraxacum, (dandelion) leaves radical runcinate, outer calyx-leaves reflexed. $\% \cdot \mathrm{y}$.

Prenanthes. Florets in a simple series (or in one circular row :) calyx calycled: receptacle naked : egret simple, sub-sessile. 49.53-alba (white lettuce) florets numerous, leaves angular and halbert-form toothed, flowers nodding. 21. w.-altissima, florets about 5 , leaves 3-lobed. $21 \cdot \mathrm{y}-\mathrm{r}$ - - cordata, leaves cordate toothed ciliate. $2 \% \mathrm{y}$.

LAcTuca. Calyx imbricate, cylindric, with the margin membranaceous: receptacle naked: egret simple, stiped: seed smooth. 49.63-sativa (garden lettuce) leaves rounded, those of the stem cordate, corymbed. y.-elongata (tall lettuce) leaves smooth beneath, lower ones runcinate clasping, upper ones lanceolate. $\% \cdot w$. Dr. Bigelow calls this the fire-weed; but the fire-weed is a species of senecio.

Hieracium. Calyx imbricate, ovate; egret simple, sessile : receptacie naked or sub-pilose._(From white becoming yellowish.) 49.53-venosum

ped egret will not appear before full maturity. Pull off all the germs; if notling is left, the receptacle is naked. But whatever is left belongs to the receptacle, and gives it the character of chaffy, hairy or bristly. 
(veiny hawkweed) scape naked branched, leaves radical prostrate obovate ciliate entire with colour. ed veins. 2l · y. - kalmü, stem many-flowered, leaves small lanceolate toothed. 2f.y.-scabrum, panicle glandular hairy. 24. y.

Sonchus. Calyx imbricate inflated : egret pilose, sessile : receptacle naked. 49.53-oleraceus (sow thistle) peduncles woolly umbelled, calyx glabrous, leaves runcinate denticulate. (-). y.-hallidlus, raceme terminal compound, leaves lance-en. siform clasping toothed. y.-halustris, calyx and peduncles bristly, somewhat umbelled, leaves runcinate with sagittate base. $\boldsymbol{\psi}$.

Cynara. Receptacle bristly: calyx dilated, imbricate ; scales with fleshy base, emarginate, pointed : egret plumose, sessile. 49. 54-scolymus, (garden artichoke) leaves prickly or unarmed pinnate and undivided, calyx-scales ovate. b.e.

Hyoseris. Calyx sub-globular, calycled, the exterior very small : receptacle naked : egret unequal, outer simple, pilose, inner membranaceous : seed near the margin often involved in the scales of the calyx. 49.53-hrenanthoides, (swine lettucc) stem. leaves clasping, radical ones sub-lyrate. $y$.

Krigia. Calyx simple, many-leaved: egret 5 membranous leaves with 5 alternating bristles : receptacle naked. 49.53-virginica, (dwarf-dandelion) scape 1 -flowered, leaves lance-lyrate, glabrous. (i). $y$.

Cichorium. Calyx calycled : egret many chaffy leaves : receptacle somewhat chaffy. 49.53 -intibus, (succory or endive) flowers sessile in pairs, leaves runcinate. $2 \%$. b.

B. Florets tubulous; flowers cafitate.

Liatris. Calyx imbricate, oblong : egret plumose, coloured: receptacle naked. (Flowcrs pur: 
ple.) 49.54-sficata (gay feather) leaves linear entire glabrous ciliate at base dotted, flowers spiked, calyx-scales linear. 24. p.-scariosa, raceme terminal, calyx-scales obovate. 4 . p.-squarrosa, peduncles 1-flowered. 24. p.

Vernonia. Calyx imbricate, ovate ; egret double, exterior chaffy and short, interior capillary: receptacle naked : stigma 2 -cleft, shortish. 49.55 - noveboracensis (flat-top) corymb fastigiate, stem glabrous, leaves lanceolate. 24. p.-fraealta, stem pubescent. 27. r.

CNicus. Calyx imbricate, with prickly scales: receptacle villose : egret plumose. (Taken from the carduus.) 49.54-lanceolatus (thistle) leaves decurrent hispid pinnatifid with 2 -lobed spreading divisions, stem hair $y$, calyx with lance-thorny scales. $\hat{o}$. p.-arvensis (Canada thistle) leaves sessile glabrous tooth-pinnatifid edged with spines, panicled, scales of calyx minute lanceolate with short soft bristly spines. $\delta \cdot p$ - -altissimus, radical leaves pinnatifid, stem leaves lance-oblong toothed ciliate. 24. p.

Carthamus. Calyx ovate, imbricate with scales, ovatish-leafy at apex: egret chaff-hairy or none: receptacle chaff-bristly. 49.54-coeruleus (falsesaffron) egret hairy, leaves thorny-toothed. 2!. b. e. tinctorius, egret none, leaves serrate prickly. .y.e.

ONopornow. Calyx imbricate with sharp-mucronate scales : egret pilose, caducous : receptacle alveolate : seed transversely sulcate. 49. 54acantrium (cotton thistle) leaves sinuate downy thorny oblong, decurrent.

CARDuUs. Calyx ovate, imbricate with prickly scales : receptacle villose : egret pilose. $49.54-$ fectinatus (comb-tooth thistle) leaves decurrent lanceolate pectinate. $\delta \cdot \mathrm{p} .-$ marianus, leaves clasp. ing, spines cliannelled doubly-thomed. 
ARCTIUn. Calyx globose, with scales hooked at apex: egret chaff-bristly: receptacle chaffy. 49. 54-latha (burdock) stem-leaves cordate. $\hat{\delta}$.p.

SPARGa NOPHORUs. Calyx sub-globose, imbricate with unequal scales recurve-spreading at the lips: receptacle naked: seed with a cup-like crown, sub. cartilagenous. 49. 55-verticillatus (crown-cup) leaves linear-setaceus whorled, about 1 -flowered.

\section{Florets tubulous; flowers discoid.}

Bidens. Calyx calycled, nearly equal; sometimes the flower is furnished with one or two radiate florets : receptacle chaffy, flat: egret 2,3 or 4 awns with reflexed and erect lateral prickles: seed 4.cornered. 49.55-WITH 1 OR 2 RADIATE FLORETs.-bifinnata, (burr-marigold or cuckold) leaves doubly-pinnate. - -chrysanthemoides, leaves oblong tapering to both ends, calyx shorter than ray. - y.-cernua, leaves lanceolate, outer calyx longer than the florets. - y.-WITHOUT RADIATE FLORETS-frondosa, outer calyx 6 times longer than the florets, lower leaves pinnate, upper ones serrate. (3). $y$-connata, stem-leaves in threes, floral ones lance-oblong, $\mathrm{P} \cdot \mathrm{y}$.

Kunnia. Calyx imbricate, cylindrical : egret plumose, sessile : receptacle naked. 49.55-eufiatoroides, (false boneset) leaves serrate, corymb compact. 24.w.-critonia, leaves entire, corymb spreading. $24 . \mathrm{w}$.

Eupatorium. Calyx imbricated (rarely simple) oblong : style long, cloven half way down: egret pilose or rough papillose : receptacle naked, 49.55 -FLORETS 6 OR MORE. - herfoliatum, (boneset, tho. roughwort) leaves connate-perfoliate clowny. 21 .w. - hurfureum, leaves petioled 4 or 5 in a whorl lance-ovate, stem hollow. 24. p.-verticillatum, leaves 3 or 4 in 2 whorl with wedge-form base 
stem solid glabrous. 24. p.-FLORETS 3 TO 5.-sCssilifolium, leaves clasping dotted beneath. 24. w. verbenifolium, leaves sessile, florets 5 , calyx hairy. 24. w.

Mrkania. Calyx 4 or 6.leaved, equal ; 4 or 6 flowered; receptacle naked: egret pilose. 49.55scandens (climbing boneset) stem glabrous, leaves cordate spread-toothed. $w$.

Cacalin. Calyx cylindric, oblong; somewhat calycled at the base : receptacle naked: egret pilose. (Leaves mostly succulent.) 49.55-suaveo. lens (wild caraway) leaves hastate-sagittate. 24.w. -atriflicifolia, radical leaves cordate, stem-leaves deltoid. 2. w.-reniformis, radical leaves repandtoothed. 24. w.

\section{Order II. Polygamia Superfuta.}

\section{A. Flovers discoid.}

Coxyza. Calyx imbricate with scales sub-linear ovate; or hemispheric and sub-scarious: egret simple, capillary: receptacle naked. Florets of the ray with a 3 -cleft border, not composing a ray in appearance. 49. 55-camfliorata, (plowman's wort) leaves lance-orate sessile, corymb crowded. O. p.-bifrons, leaves spatulate clasping. 4 .

Gnaphalium. Calyx imbricate, with the mar. ginal scales rounded, scarious, shortish, glossy, coloured : receptacle naked : egret pilose or plumose. (Florets often all perfect.) 49.5.5-12lantagineum, (mouse-ear, life everlasting) small, corymb compact, root-leaves spatulate, shoots procumbent, dioecious. 21. W.-margaritaceum, (common life everlasting) leaves lance-linear acuminate, corymb fastigiate, odour pleasant. 21.w.-uliginosum, (cudweed) stem much-branched, leaves lance-linear tapering to both ends, racemes terminal crowded. D. w. 


\section{CLASS XVIII, ORDER II.}

Artemisia. Calyx imbricate, with scales rounded, converging: egret 0 : receptacle somewhat villose or nakedish. (Flowers mostly rounded.) 4?. 55-LEAYES PINNATIFID-absynthium (wormwood) divisions of radical leaves obtuse, of stem-leaves acutish. 2!. e.-vulgaris, divisions of leaves all acute and toothed. 24.-LEAvEs PINNATE-abrotomum (southern wood) lower leaves doubly-pinnate, upper ones pinnate filiform. h.e.-prontica, stemleaves doubly-pinnate with linear leafets, flowers nodding. $\%$.

TANACETEM. Calyx inbricate, hemispheric: scales acuminate : rays obsolete, 3-cleft : egret somewhat marginal : receptacle naked. (Flowers corymbed.) 49.55-vulgare, (tansy) leaves doub. ly-pinnate. 24. y.e.

\section{B. Flower's radiate.}

Chrmsanthemem. Calyx hemispherical, imbricate, with the scales membranous at the margin : receptacle naked : egret none, or a narrow margin. 49. 55-leucanthemum, (ox-eyed daisy) leaves clasping obtuse cut-pinnatifid, radical ones obovate. $\boldsymbol{\psi}$. w.-harthenium, (feverfew) corymbed, leaves compound, leafets ovate. 2\%. e.

TAGetes. Calyx simple, 1-leafed, 5-toothed, tubular : florets of the ray 5, permanent : egret 5 erect awns: receptacle naked. 49. 55-erecta, (African marigold) stem erect, calyx angular, leaves pinnate. 24. y. e.-hatula, (French marir gold) stem spreading, calyx smooth. $\boldsymbol{\Psi}$. y. e.

BeLris. Calyx hemispherical, scales equal : egret 0 : receptacle naked conical : seed obovate. 49.55-nerennis, (garclen daisy) scape 1-flowered, leaves obovate. \&. b-w. e.

Pyrethrum. Calyx hemispherical, imbricate with scales acutish and scarious margined: egret 
a marginal ring: receptacle naked. (Taken from Chrysanthemum.) 49.55-serotinum, (wild feverfew) leaves lanceolate, branches corymbed. 4 .

ArNica. Calyx with leafets equal : egret simple : receptacle naked. The florets of the ray usually have 5 filaments without anthers. 49.55montana, (arnic) leaves ovate entire having 2 opposite cauline ones. $24 \cdot \mathrm{y} \cdot \mathrm{e}$.

Doronicum. Calyx-scales equal, in two rows, longer than the disk: egret simple : receptacle naked: seeds of the ray naked and destitute of egret. 49.55-nudicaule, (leopard's bane) stem almost leafless. 4 . $\mathrm{y}$.

Inula. Calyx imbricate or squarrose: egret simple : anthers ending in 2 bristles at the base : receptacle naked : ray florets numerous. 49.55-ha. lenium, (elecampane) leaves clasping ovate downy beneath, calyx-leaves ovate. $21 \cdot y$-mariana, leaves sessile lance-oblong glandular-toothed tapering base. 2. y.

ERIGERON. Calyx imbricate: egret pilose: receptacle naked : florets of the ray linear, very narrow, numerous. 49.55-bellidifolium, (early fleabane) root-leaves obovate serrate, stem-leaves lanceolate entire. 24. p. w.-hihiladelfhicum, (blue flea-bane) stem-leaves half-clasping lanceolate subserrate, ray-fiorets as long as those of the disk, stem many-flowered. 2 • p-b.-canadense, (Canada flea-bane) leaves lanceolate ciliate, lower ones tooth-serrate, panicled, stem and flowers hairy, flowers small numerous. (-). w.

SolIDAGo. Calyx-scales imbricate, closed : egret simple : receptacle naked, furrowed with dots or punctures: ray-florets about 5. (Yellow.) 49. 55--RACEMES POINTING ONE WAY.--allissima, (golden rod) stem erect hairy tall, leaves lanceolate rough wrinkled, lower ones deep-serrate, raceme 
panicled. 2\%.y.-rugosa, very rough, lower leaves with close serratures, racemes spread-panicled. $y$. -canadensis, stem downy, leaves rough serrate, racemes panicled recurved, ligulate, florets abbreviated. 21. y.-RACEMES ERECT.-bicolor, leaves oval, lower ones serrate, stem and leaves hairy, calyx-leaves obtuse. 4. w-y.-lanceoluta, stem and leaves glabrous, branching, leaves lance-linear entire, corymbs terminal. 21. y.-laevigata, stem and leaves smooth, leaves fleshy, peduncles scaly downy. 4. y-caesia, (a variety of flexicaulis) glabrous, leaves acuminate, racemes axillary. y.

Senecio. Calyx cylindric; leafets with withering, blackish tips, and a few small calycle leafets at the base : egret simple : receptacle naked. 49.55 -aureus, (rag-wort) root-leaves heart-ovate serrate petioled, stem-leaves pinnatifid-toothed with lanceolate terminal divisions. 24. y. -hieracifolius, ray obscure, leaves clasping oblong unequally toothed. (). y.-vulgaris, ray obscure, flowers scattered, leaves sinuate-pinnatifid clasping toothed. (-). y.-elongatus, glabrous, root-leaves spatulate, stemleaves pinnatifid. $2 \%$

Tussilago. Calyx simple, scales equal, and equalling the disk, sub-membranous: reccptacle nalsed: pistillate florets ligulate or without teeth : egret simple, sessile. (Sometimes polygamous.) 49. 55-farjara, (colt's foot) scape 1-flowered scaly, at length come out radical heart-angular leaves. 2. $y$.

Aster. Calyx imbricate, the inferior scales spreading : egret simple : receptacle naked, scrobiculate. (Florets of the ray more than 10, except in solidaginoides, and a few other species.) 49.55. Leaves lance.linear entire-D-solidaginoides, (star-flower) leaves obtuse with scabrous margins, branches fastigiate-corymbed, flowers sessile with 
5-flowered rays, calyx-scales whitish green-tipped. 4. w.-linarifolius, leaves keeled dotted rough cus. pidate, fastigiate, stem sub-decumbent. p.-subulatus, glabrous, leaves subulate, stem panicled, flowers small many. w.b. - salicifolius, glabrous, stem panicled at top, calyx-scales acute with spreading tips. b. -novae-angliae, leaves clasping eared at the base hairy, calyx-scales lax coloured lanceolate. p.-cyaneus, leaves clasping smooth, stem panicled glabrous, branches racemed, inner calyx. scales with coloured tips. b.-LEAvEs ovatish xNTIRE-cornifolius, glabrous, leaves acuminate short-petioled with rough margin.w.-amygdalinus, leaves tapering to the base, margin rough, fastigiate. W. LEAvES SERRATE CORDATE-undulatus, leaves clasping, lower ones with winged petioles. b. - cordifolius, leaves all with winged petioles. pw. LEAVES SERRATE OR TOOTHED OVATE.-COrymbosus, fastigiate. W.-chinensis, (china aster) calyx leafy, branches 1 -flowered.e. Leaves LANCEOLATE, LOWER ONES SERRATE-funiceus, leaves clasping, panicled-diffusus, leaves glabrous, stem. hairy, much-branched. w.

Helenium. Calyx 1-leafed, many parted : egret 5 chaffy leaves: receptacle naked in the disk, chaffy in the ray: florets of the ray half-3-cleft : seed villose. (Leaves decurrent.) 49.55-autumnale, (false-sunflower) leaves serrate glabrous. 4. y.

Boltonia. Calyx imbricate: egret tooth-awned, somewhat 2.horned : receptacle hemispherical, alveolate: florets of the ray numerous : seed flatcompressed, margined. 49. 55-asteroides, (falsechamomile) leaves entire, seeds oval glabrous. $2 \%$ r-y.-slastifolia, lower leaves serrate, seeds obcordate. 24. r-y.

Matricaria. Calyx flat, imbricate, scales scarious at the margin: receptacle naked, cone-cylin- 
aric: egret none. 49.55-chamomilla, (wild chamomile) leaves pinnate and doubly-pinnate.

Anthemss. Calyx hemispherical, nearly equal: egret 0 , or a membranous margin : florets of the ray more than $5:$ receptacle chaffy, the chaffs fat, with a rigid acuminate apex. 49.55-cotula, (mayweed) receptacle bristly, leaves doubly-pinnate, leafets 3-parted. O. w-arvensis, divisions of leaves lance-linear. $($ ) w.-nobilis, (chamomile) receptacle chaffy, leafets 3-parted linear-subulate. 21. e.

Achillea. Calyx imbricate, ovate: egret 0 : receptacle chaffy: florets of the ray 4 to 10 , dilated. (Flowers corymbed.) 49. 55-millefolia, (yarrow) leaves doubly-pinnatific, stem groved. 21.w.

Verbesina. Calyx in double order : receptacle chaffy: florets of the ray about 5 : egret awned. 49. 55-sigesbeckia, (crown-beard) leaves decurrent opposite lance-ovate serrate. $2 \cdot y$.

Order III. Polygamia Frustranea.

RUdBeckia. Calyx with a double order of scales : receptacle chaffy, conic : egret a 4-toothed margin or 0.49 .55 -laciniata, (cone flower) leaves pinnate, stem-leafets oblong, with the egret. 24. y. - Inurfurea, leaves lance-ovate alternate undivided, ray-petals 2-cleft. 24.p.-triloba, leaves spatulate, lower ones 3 -lobed, upper ones undivided. $\left\{\begin{array}{c}y \\ \text {. }\end{array}\right.$

Helianthus. Calyx imbricate, sub-squarrose, leafy : receptacle flat, chaffy : egret 2-leaved, caducous. 49. 55-annuus, (sunflower) leaves all cordate 3-nerved, flowers drooping. (-).y.-decafetalus, leaves ovate, stem smooth below, peduncles scabrous, ray about 10-petalled. 2\%.y.-divaricatus, leaves opposite sessile ovate-oblong, panicle forkeả. 2 . $\mathrm{y} \cdot$ - altissimus, leaves alternate lanceolate, petioles ciliate. 24. y-tuberosus, (Jerusalem artiI. 2 
choke) lower leaves heart-ovate, root tuberous: 4. $y$.

Coneorsis. Calyx double, also many-leaved: seeds compressed, emarginate : receptacle chaffy: egret 2-horned. 49.55.-verticillata, (tickseed sunflower) leaves pinnate three or five in a whorl. 2\%. w.-trichostzerma, pinnate leaves with 5 or 7 leafets, seed wedge-form. $y$ - -auriculata, leaves ovate entire, lower ones ternate. $4 . y$.

Centaurea. Calyx various, mostly imbricate, roundish : egret simple, various: receptacle bristiy: corols of the ray funnel-shaped, longer, irregular. 49.54-cyanus, (blue-bottle) leaves linear entire lower ones toothed. (-).b.e.-jacea, leaves lanceolate entire, radical ones a little toothed. 4. b.beneclicta, (blessed thistle) leaves sub-decurrent, caly $\mathrm{x}$ with branchy thorns. - centaureum, (tall bluebottle) leaves pinnate, leafets doubly-serrate. e:

\section{Order IV. Poltgamia Necessaria.}

Silphivar. Calyx squarrose, scales leafy, broad : egret 2-horned-margin: receptacle chaffy: seeds compressed, obcordate, margined. 49.55-herfoliatum, (ragged cup) leaves opposite connate, stem 3sided. 24. y.-ternatum, leaves in threes near the middle of the stem, panicled. 24. $y$.

Polymina. Calyx double, exterior 4-5-leaved, interior 10-leaved, leafets concave : egret $0: \mathrm{re}-$ ceptacle chaffy. 49. 55-canadensis, (leaf-cup) leaves denticulate, lower ones pinnatifid. 24. w.uvedalia, leaves 3 -lobed. $2 \%$. y.

Iva. Calyx 3-leaved: styles 2, long: seed naked, obtuse : receptacle hairy : ray 5 -flowered. 49. 55-frutescens, (highwater shrub) leaves lanceolate rough-dotted deep-serrate. h. $g$. 
Order V. Polygamia Segregata.

Elephantopus. Common calyx 3-leaved, partial calyx 4-flowered : florets ligulate: receptacle naked : egret setaceous. 49.55-carolinianus, (elephant foot) stem simple pilose, leaves narrowed at the base. 4 . p. Southern states.

\section{CLASS XIX. GYNANDRIA.}

Order I. Monandria-or Order II. Dian: DRIA.

Calyx 5-leaved coloured, (or corol 5-petalled.)

A. With a stur under the lower lif.

Orcuis. Calyx ringent-like, the upper leafets vaulted: lip dilated, spur long: anther terminal adnate. 7.21-sfrectabilis, (scarlet orchis) lip of nectary emarginate, spur as long as germ, stem leafless. r. - orbiculata, (round-leaved orchis) lip entire, side-leafets of calyx spreading, spur longer than germ. g.-fimbriata, lip 3-parted, divisions wedge-form fringed, spur longer than germ. \%. p. hrsychodes, lip 3-parted fringe-like, spur as long as germ. 4. y-g.-ciliaris, lip 3-parted lanceolate fringed, root-bulb undivided, spur long, 4 . y.

Lmodorux. Calyx somewhat spreading: lip spreading, elongated at the base into a spur: anther a terminal lid, caclucous. 7. 21-unifolium, (limodore) has but one leaf. w.

Satyrium. Calyx ringent-like, upper leafet vaulted: lip linear, parted or sub-entire: spur short, sub-inflated and somewhat 2-lobed : anther terminal, adnate. 7.21 -bracteatum, (satyrion) lip bifid at the apex, bract twice as long as the flow: el. g. 
B. Lower lif quithout a shur.

Neotria. Calyx ringent-like, the outer side leafets united before about the inflated base of the lip: anther parallel to the acuminate style, affixed behind. 7. 21-zubescens, (ladies' traces) radical leaves ovate netted with white veins, scape and flowers hairy, spike oblong. 2/.w.-cermua, rootbulbs branched, stem leafy sheathed, lip of nectary entire, spike spiral. 24.w.-iortilis, lip 3-lobed, middle division crenate, spike spiral, leaves radical linear. w.-aestivalis, stem leafy.

Epipactis. Calyx erect, spreading: anther a permanent lid: lip flat, pendulous, 2-cleft at the apex. 7.21 -convallaroides, (lily orchis) stem with 2 roundish cordate leaves, lip obtuse 2 -lobed.

Cymbidium. Calyx 4 or 5-leaved, erect or spreading: lip concave at the base, with a spreading border : anther a deciduous lid: pollen globose. 7. 21-corallorhizon, (corol root) lip oblong acute undivided, stem leafless, sheathed. $y-w$. Plant white-odontorhizon, lip ovate obtuse, stem leafless sheathed. w.p. Plant white-fzulchellum, (grasspink) radical leaf ensiform, lip erect with expanded border. 24.p.-hyemale, leaves radical 5-nerved, lip obovate with crenate margin. $y-g$.

Malaxis. Calyx spreading, turned upside down by the twisting of the peduncle when the petals open : lip ascending, concave, spreading: anther a lid. 7.21-liliafolia (marsh-twayblade) leaves 2ovate-lanceolate, lip wedge-obovate pointed. 4 . p.y.-ophiogrlossoides, leaf 1 heart-ovate, lip 2-cleft. 21. w.

Arethusa. Calyx somewhat ringent-like; leafets somewhat converging : anther a permanent lid : pollen powdery-granulated. 7.21-bulbosa (arethusa) leafless, 1-flowered, root globose. 24.p.ophioglossoides, scape 1 or 3 -flowered with 2 leaves, root fibrous. $4 \cdot \mathrm{p}$. 


\section{Order II. Diaxdria.}

Crpripedium. Calyx coloured, 4-leaved, spreading: lip inflated, hollow : style with a terminal lobe; and a petal-like appendage on the upper side. 7.21-fiubescens (yellow ladies' slipper) stem leafy, style-lobe obtuse, shoe-form nectary shorter than calyx. 21. y. - shectabile (ladies' slipper) stem leafy, style-lobe obtuse, nectary longer than calyx: 2'. p.-humile, stemless, leaves radical. 4. P.

\section{Order V. Pentandkia.}

Ascrepias. Follicles 2: nectaries 5, concave, containing a little horn: petals reflexed. 30.47Stems SiMple, Leaves opposite-syriaca, (milkweed, or silk-weed) leaves lance-oblong sharp downy beneath, umbels downy. 21.w-p.-quadrifolia, four largest leaves near the middle of the stem. 4 . w-r. - hurhurascens, leaves ovate downy beneath, nectaries upside down, umbels erect. $2 \% \cdot \mathrm{p}$-obtusifolia, leaves clasping round-obtuse oval-oblong. 4. P.-STEM SIMPLE, LEAVES WHORLED OR SCATTERED-verticillata, leaves mostly whorlec narrowlinear. 2l-y-w.-tuberosa, leaves scattered lanceoblong rough-haired. 24. -incarnata, stem branching, leaves lanceolate, hairy. 21. $\mathrm{r}$.

Cyanchum. Calyx 5-toothed : corol wheel form: nectary cylindric, 5 -toothed : follicles 2.30. 47-obliquum (choak-dog) twining, leaves cordate, corymbs axillary. p.

\section{Order Vi. Hexandria.}

Aristolochia. Calyx 0: corol superior, 1-petalled, ligulate, inflated at the base : capsule 6.celled, many-seeded. 11.23-serhenlaria (birth-wort) stem zigzag, scaped, lips of corol lanceolate. 4 .- 
sifho, stem twining, peduncles bracted, limb of corol s-cleft. $z \cdot y$.

* Arum was formerly placed here, but is now removed to class monoecia.

\section{CLASS XX. MONOECIA,}

\section{Ordeir I. Monandra.}

Fluvialis. Staminate flowers-calyx 0 : corol $0:$ anther sessile. Pistillate flowers-calyx $0:$ co. rol 0 : style filiform : stigma 2-cleft : capsule 1seeded. 15.6. (Flower's axillary.)-flexilis, (rivernymph) leaves in sixes linear 2-toothed at the apex. (3)-fragilis, leaves in threes or opposite pricklytoothed. :-

Chara. Staminate flowers-calyx 0: corol 0: anther globose, sessile. Pistillate flowers-calyx 0 : corol 0 : style 0 : stigmas 5 : berry 1-celled, many-seeded. (Aquatics.) 15.6-vulgaris (chara) branclilets terete, joints leafy, leafets oblong-subulate-foliosa, the leafets linear.

Najas.-Staminate flowers-caly x 2-lobed : corol 4-cleft: anthers sessile, cohering. Pistillate flowers-calyx 0 : corol 0 : style subulate : stigma 2 or 3-cleft: capsule 1 to 4-seeded. 15.6-canadensis (water nymph) small filiform smooth, leaves narrow-linear.

\section{Order II. Diandita.}

LEMxa. Staminate flowers-calyx 1-leafed: corol 0. Pistillate flowers-caly 1 1-leafed : corol 0 : capsule 1-celled, 2-seeded, 54,6-minor (duckmeat) leaves oval flat both sides cohering at their bases, root solitary - polyrliza, leaves oval flat cohering 2 or 3 together, roots fascicled. Leaves greenish-purple; appear like flaxseed, scattered upon the surface of stagnant watcrs, with the roots 
srowing immediately from the under surface of the leaves, and the flowers from their upper surfaces. Found in great abundance near Williams' College.

Podostemum. Staminate flowers-calyx 0: corol 0 : stamens affixed to a pedicel. Pistillate flowers-calyx $0:$ corol $0:$ germ ovate : stigma 1 , sessile : capsule 2-celled, 2-valved, many-seeded. 54.6 - ceratofihyllum (thread-foot) stem filiform floating, leaves pinnate, flowers axillary.

\section{Order III. Triandria.}

Trpha. Ament cylindric. Staminate flowerscalyx obsolete, 3-leaved : corol 0 . Pistillate flowers-bclow the staminate : calyx 0 : corol 0 : seed 1 , pedicelled; the pedicels surrounded at the base with long hairs resembling egret. 3.8-latifolia (cat-tail, or reed mace) staminate and pistillate spikes near or adjoining, leaves sub-ensiform. 24. -angustifolia, staminate and pistillate spikes remote, leaves half-cylindric. 4 .

Sparganium. Staminate flowers-ament roundish : calyx 3-leaved: corol 0. Pistillate flowerscalyx 3-leaved : corol 0: stigma 2-cleft: drupe juiceless, 1-seeded. 3. S-ramosum (burr-reed) leaves 3 -sided at the base, common peduncle branched. 2\%. g-w.-simfllex, common peduncle simple. 4 .

$\mathrm{ZEA}_{\mathrm{EA}}$ Staminate flowers-calyx-glume 2-fiowered, awnless : corol-glume awnless. Pistillate flowers-calyx-glume 2-valved: style 1, filiform, pendulous : seed solitary, immersed in an oblong receptacle. 4. 10-mays (Indian corn) leaves entire linear-lanceolate keeled. (-).g. Var. hrecox (summer corn) small and ripens in the summer.

Tripsacum. Staminate flowers-calyx-glume 2-flowored, outer one staminate, inner one neutral: corol-glume membranaccous. Pistillate flowers- 
calyx-glume 1-flowered, covered with a 1-leafed involucre perforated with hollows: corol-glume 2valved: styles 2 : seed 1. 4. 10-dactyloides (sesame grass) spikes ternate. 24 .

Corx. Staminate flowers-in remote spikes: calyx-glumes 2-flowered, awnless : corol-glume awnless. Pistillate flowers-calyx-glume 2 -flowered: corol-glume awnless: style 2-parted : seed covered with the bone-like calyx. 4. 10-lacryma (Job's tear) culm branched, leaves wrinkled, seeds ovate.

CAREx. Aments imbricate (usually in cylindric spikes.) Staminate flowers-calyx-scales solitary: corol 0. Pistillate flowers-calyx-scales solitary : corol inflated, monopetalous, 2-toothed at the apex : stigmas 2 or 3 : nut 3 -sicied, inclosed in the permanent corol, which becomes an utriculus-like permanent aril. 3. 9-STIGMAS 2, STAMENS AND PISTILS IN DISTrNCT SPIKES-coesfitosa† (bog sedge) pistillate spikes about in threes, lower one very short, aril longer than the obtuse scale-crinita, staminate spikes in pairs, pistillate spikes in fours, aril shorter than the awned scale-Stigmas 2, SPIKES SEVERAL, ANDROGYNOUS, WITH STAMINATE FLORETS ABOVE-stifiata, aril bi-cuspidate planoconvex nerved, culm very rough, spikelets oblong about in fives-paniculata, aril with ciliate-serrate margin, spikes panicled.-LIKE THE LAST, EXCEPT WITH PISTILlATE FLORETS ABOVE-scirfoides, spikelets about in fours, aril ciliate-serrate- festu. cacea (fescue sedge) spikelets about in eights, aril ciliate-serrate.-STIGMAS 3, SPIRES ANDROGYNOUS WITH STAMINATE FLORETS ABOVE- hedunculata,

† Fifty-five species of Carex have been examined and described in the Northern States. It is therefore very difficult to select the most common, in conformity with our plan.We have inserted none but those found most common near Yale College by the author, and near Williams' College by Professor Dewey. 
spikes about in fours, aril oborate $3 \cdot$ sided obtuseSTIGMAS 3 , STAMENS AND PISTILS ON DISTINCT SPIKES, STAMIXATE OXES SOLITARY, PISTILLATE ONES SESSILE OR WITH INCIOSED PEDUNCIESvaria, pistillate spikes short about in threes, aril shorter than the scale-icniaculata, pistillate spilies in threes, aril a litte longer than the scale-lufue linc, pistillate spikes in thress, aril mucronate several times longer than the scale-oligocarna, pistillate spikes in pairs, aril oborate beaked longer than the scale-folliculata, pistillate spike sub-sotilary about 6.flowered.-LIRE THE LAST, EXCEPT WITH LONG PEDUXCLES AND LONG SHEATHSsilantaginea (plantain sedge) pistillate spikes in fours, culm with leaf-pointed bracts, radical leaves broad 3-nerved-granuluris, pistillate spikes in threes, aril nerved globular inflated sub-emarginate, scale lance-orate-conoidea, pistillate spikes in pairs, aril oblong-conic obtuse equalling the awned scale.-Stigmas 3, staminate spikes maxtflellita, staminate spilies in pair's, aril bifid, shorter than the cblong anned scale-lacustris, staminate spikes in fours, aril 2-forked. A variety, gigantea, is very large-r'ecicaria, staminate spikes in threes, aril oblong inflated bi-cuspidate.

SCLERia. Staminate flowers-calyx-giume 2 or 6 -valved, many flowered : corol-glumes awnless. Pistillate flowers-calyx like the staminate: corol 0 : stigmas 1 to 3 : nut coloured sub-globose. S.9. - verticillata (whip.grass) flowers spiked, culm 3. sided glabrous, fruit flobose.

Comptoxia. Staminate flowers-ament cylindric with calyx-scales : corol 2-petalled: filaments 2 -forked. Pistillate flowers-spike or ament ovate, corol 6-petalled: styles 2: nut ovate. 50,99-asflenifolia (sweet-fern) Icaves alternately pinnatifid hairy beneath. h. $g$. 
Serpicula. Staminate flowers-calyx 4-toothed: corol 4-petalled. Pistillate flower's-calyx 4parted : nut tomentose. 15.88-verticillata (little snake-weed) leaves whorled prickly serrate. w.

Eriocaulon. Common calyx many-leaved : florets many in an imbricate head: corol 3 to 6 petalled: stamens higher than the germ: capsule 3-celled: seed 1, crowned with the corol. Central flowers staminate. 6. 13-kellucidum (pipe-wort) stem 6 or 7 -angled, stem and root pellucid, leaves awl-form 5-nerved. Grows mostly under waterdecangulare, stem 10-angled-gnaphaloides, culm sub-compressed.

Order IV. Tetrandria.

Boehmeria. Staminate flowers-calyx 4-parted : corol 0 : nectary 0 . Pistillate flowers-calyx 0 : corol 0 : styles 2: seed 1, compressed. (Flowers capitate.) 53.98-cylindrica (round-head) leaves opposite glabrous. 4 -lareriflora, leaves alternate rough. 4 .

URTICA. Staminate flowers-calyx 4-leaved: corol 0: nectary central cyathiform. Pistillate fowers-calyx 2-leaved (2-valved :) corol 0 : seed 1 , glossy. 53.98-dioicu, (nettle) leaves opposite cordate coarse-serrate, spike-panicled, dioecious. $\boldsymbol{4}$. g.-urens, leaves opposite oval 3 to 5-nerved, racemes simple. G. - fumila, leaves opposite ovate, racemes 2 -parted shortish sub-corymbed. - procera, leaves opposite cordate, petioles ciliate. 21.-canadensis, leaves alternate heart-ovate, aments branched 2-ranked erect. 4.-zuhitlowi, (Albany flax) leaves alternate heart-ovate 3-nerved, upper ones opposite, panicle forked, root tuberous. $2 \%$. About 6 feet high. Discovered near Albany by Mr. Charles Whitlow.

Parietaria. Polygamous. Perfect flowers- 
calyx 4-cleft inferior: corol 0 : stamens elastic : style 1: seed 1. Pistillate flowers-calyx 2-leaved: seed covered with the dried elongated calyx. 53. 98-hensylvanica, (pellitory) involucre longer than the flower, leaves lance-oblong with opake dots. 21.

Mores. Staminate flowers-calyx 4-parted: corol 0. Pistillate flowers-calyx 4-leaved : corol 0: styles 2: caly $\mathrm{x}$ berried: seed 1. 53.98-alba, (mulberry) leaves heart-oblique smooth. h.w.rubra, leaves cordate downy beneath. $h$. w.

A lxus. Staminate flowers-ament composed of wedge-form truncate 5 -flowered receptacles : calyx a scale : corol 4-parted. Pistillate flowerscalyx 2 -flowered scales : corol 0 : sced compress. ed ovate wingless. 50.99-serrulata, (alder) leaves cbovate acuminate, stipules oval obtuse. h.g.

Buxus. Staminate flowers-calyx 3-leaved : petals 2: germ a mere rudiment. Pistillate flowers-calyx 4 -leaved: petals 3 : styles $3:$ capsules 3-beaked, 3-celled : seeds 2.38.96-sempervirens, (box) leaves ovate. h. Var. angustifolia, leaves lanccolate. Var. suffruticosa, small, Icares obovate.

\section{Order V. Pentandria.}

Xavthius. Staminate flowers-common calyx imbricate : corol 5-cleft, funnel-form: receptacle chaffy. Pistillate flowers-involucre 2-leaved, 1. flowered: corol 0: drupe dry, muricate, 2-cleft : nut 2.celled-strumarium, (sea burdock) stem unarmed, leaves cordate 3-nerved, fruit an oval burr. - irientale, leaves 3 to 5 -lobed.

Amerosia. Staminate flowers-common calyx 1-leaved : corol 1-petalled, 5-cleft, funnel-form : receptacle naked. Pistillate flowers-calyx 1-leafed, entire, the swelling part 5 -toothed, 1 -flowered : corol 0 : nut from the indurated calyx, 1 -seeded. 54. 


\section{CLASS XX. ORDERS VI AND XIII.}

32-elatior, (common hogweed) leaves doubly-pin: nate smoothish with ciliate petioles, racemes terminal panicled. - -artemisifolia, branches fastigiate. B.-trifica, leaves 3-lobed, fruit 6-spined.

Amanartues. Staminate flowers-calyx 3 or 5 . lcaved: corol 0 : stamens 3 or 5 . Pistillate flowers. - calyx and corol as the staminate: styles 3 : capsule 1-celled, opening transversely: seed 1. 54 . so-oleraceus, (pot amaranth) glomerules axillary ramose, leaves rugose oblonis very obtuse emarginate. (- g.-hybridus, leaves lance-ovate, racemes decompound crowded erect. (-) g-p.-hypochondriacus, leaves lance-oblong mucronate. 6. p.albus, glomerules axillary, leaves obovate retuse, stem 4-sided. 3.-melancholicus, (love-lies bleeding) glomerules axillary peduncled, leaves lanceovate coloured. $\Theta$. p. e.

\section{Order VI. Hexandria?}

ZizANia. Staminate flowers-calyx 0 : corolglume 2-valved, awnless, with pistillate flowers intermixed. Pistillate flowers - calyx 0 : corol-glume 2-valved, cuculate, awned: style 2-parted: seed 1 invested in the plaited corol. 4. 10-clavulosa, (water oats) panicles branching, pistillate flowers above, bract clavate.

\section{Order XiII. Polyandria.}

Myriophyludr. Staminate flowers-calyx 4cleft: petals 4 , callucous : stamens 4,6 or 8 . Pistillate flowers-calyx and corol like the staminate: pistils $4:$ style $0:$ secels 4 , having a bark. 15.6-ficatum, (water millfoil) leaves all pinnate capilla1y, lower ones whorled, all under water. 4 .

SAgrfTaria. Staminate fowers-calyx 3-leared : corol 3.petalled : filaments mostly 24. Pistillate flowers-calyx and corol as in the staminate: 
pistils many : capsules aggregate, 1.seedecl. 5.13 - sagittifulia, (arrow-head) leaves lanceolate acuminate sagittate with strait lanceolate hind lobes. 2\%. w.

Calla. Spathe ovate becoming expanded: spadix covered with the fructification: stamens intermixed. Staminate flowers-caiyx and $\operatorname{corol} 0$ : anthers sessile. Pistillate flowers-calyx and corol 0 : berries 1-celled crowned with the short style. 2. 7-falustris, (water arum) leaves cordate, spathe ovate. 24 . w.

Arum. Spathe cuculate: spadix not entirely covered with the fructification ; being more or less naked above, with pistillate flowers bencath and staminate in the middle; (sometimes a fery are staminate beneath:) berry mostly 1 -seedod : rencrally cirrose-glandular beneath. 2.7-trikhyllum, (Indian tumip) leaves ternate, with ovate entire leafets, naked part of spadix very long clavate. 2! . p. y.g. Near Williams' college mostly dioecicus. -virginicum, leares heart-sagittate acute with obtusish hind lobes, naked part of spadix very short. In wet places.

Potenius. Staminate flowers-calyx 4-leaved: corol 4-parted : stamens 30 to 50. Pistillate flow. ers-calyx and corol like the staminate : pistils 2: berry from the indurated tube of the corol. 54.92 -sanguisorba, (burnet) stem sub-angled, filaments exsert. 4. e.

Quercus. Staminate flowers-calyx sub-5cleft: stamens 5 to 10. Pistillate flowers-calys 1-lealed, entire, scabrous, (a woody cup:) styles 2 to 5 : nut coriaceous, surrounded at the base by the permanent calyx. h. 50. 99-LEAves OBOvate, toothed - montana, (rock-oak, of chesnut oak) leaves nearly equal-toothed - bicslor, (swamp oak) leaves unequally toothed with entire basc.K 2 
Leares lanceolate toothed.-casianea, (yellow oak) leaves acuminate-LEAvEs 3-LOBED AT THE EXD-nigra, (black jack) leaves wedge-form. -Leaves sinuate with Mucronate loees, obLoxG-discolar, (two-coloured oak) downy leaves. -rubra, (red-oak) leaves glabrous obtuse, with acutish lobes, calyx flat.-coccinea, (scarlet oak) leaves glabrous deep-sinuate, calyx with tapering base.-halustris, (pin oak) leaves glabrous deep-sinuate downy at the axils of the veins, calyx with flatturbinate base.-DIFFER FROM THE LAST IN BEING oBovate.-tinctoria, (black oak) leaves pubescent beneath with obsoletely toothed lobes-ilicifolia, (scrub oak) leaves 3 to 5 -lobed downy beneath.LeAves SINUATE WITH aWnLess Lobes-alba, (white oak) leaves downy with entire obtuse lobes. Juglans. Staminate flowers-ament imbricate : calyx a scale : corol 6-parted : filaments 4 to 18 . Pistillate flowers-calyx 4.cleft superior : corol 4cleft : styles 2 : drupe coriaceous or woody : nut sulcate. $2.50 .94-L E A F E T S 7$ OR UNDER, ACUMIvAтE- -alba, (hicliory) leafets scabrous pubescent bencath lanceolate, fiuit sub-quadrangular.-squamosa, (shag-bark walnut) leafets soft-pubescent lance-oblong, fruit ovate-glabra, (pignut) leafets ovate glabrous with resinous dots beneath, fruit obcordate.-Leafets 9 or over-nigra, (black walnut) leafets lanceolate sub-pubescent, fruit globular.-amara, (bitter nut) leafets lanceolate acuminate about 9 , odd one sub-sessile, putamen thin.cinerea (butter-nut) leaves lance oblong with soft pubescence and viscous petioles, fruit oblong.

Fagus. Staminate flowers-ament roundish : calyx 5-cleft, bell-form : stamens about 12 . Pistillate flower's-calyx 4-toothed, setose : germs 2 : nuts 2 inclosed in the calyx becoming coriaceous echinate. 50.99-ferrusinea (beach) leaves ovate 
serrate acuminate pubescent beneath. $h \cdot g \cdot w,-$ sylicatica, leaves glabrous obsoletely serrate. $h$.

C.astaxea. Polygamous. Staminate flowersament naked, linear : corol 5 -petalled : stamens 10 to 20. Pistillate flowers-calyx 5 or $6 \cdot$ leaved, muricate : germs 3 : stigmas pencil-form : nuts 3 , with coriaceous putamen, inclosed in the calyx becoming echinate. 50.:9-vesca-americana, (chesnut) leaves lance-oblong giabrous, sinuate-serrate. h.

Betula. Staminate flowers-ament imbricate, scales peltate 3 -flowered : calyx a scale : stamens 10 to 12. Pistillate flowers-calyx a 2.flowered scale : sced 1, winged. 50.99-nafryracea, (canoe birch, or paper birch) leaves ovate acuminate dou. b! $y$-serrate, veins hirsute beneath. h.-hohulifolic, (white birch, broom birch) leaves deltoid long acuminate very glabrous. h.-lenta, (black birch) leaves heart ovate sharp-serrate acuminate, petioles and nerves hairy beneath. $h$. A variety, lutea, (yellow birch) bark yellowish.

Carpinus. Ament imbricate. Staminate flow. ers-calyx-scales ciliate : stamens 10. Pistillate flowers-calyx-scales 2-flowered : corol 3-cleft : nut ovate sulcate. 50.99-americana, (hornbeam) scales of the pistillate ament 3-parted with the intermediate division lance-ovate, leaves unequally serrate. $\eta_{\text {. }}$

Ostrya. Ament imbricate. Staminate flowers - caly $\mathrm{x}$ a scale : filaments ramose. Pistillate flowers-ament naked: capsules inflated, imbricate, with one seed at its base. 50.99-virsinica, (hop hornbeam) leaves oblong-ovate acuminate. $h \cdot g$. Corylus. Staminate flowers-ament imbricate: caly $\mathrm{x}$ a scale: stamens about 8 . Pistillate flowers. - calyx 3-parted, lacerated: styles 2 : nut ovate, surrounded by and included in the permanent leaf- 
like calyx. 50. 99-americana, (common hazlenul) caly $x$ of the fruit bell-form with a dilated border, leaves cordate acuminate. $h \cdot g \cdot$-rostrata, (beaked hazlenut) calyx of the fruit elongated into a tubular beak, leaves oblong-ovate. $h_{2} . g$.

Platanus. Ament globose. Staminate flowers -corol scarcely apparent, anthers growing around the filaments. Pistillate flowers-calyx manyleaved : style with a recurved stigma : seed loundish, crowned with the mucronate style, with egretlike hairs at the base. 50.99-occidencalis, (buttonwood, or plane tree) leaves quinquangular pubescent. h. g.

Liquidambar. Staminate flowers-ament conic, surrounded with a 4 -leaved involucre: filaments numerous. Pistillate flowers-ament globose, surrounded with a 4 -leaved involucre : calyx 1.leafed, pitcher-form, 2-flowered : styles 2 : capsules 2, surrounded at the base by the calyx, 1 celled, many-seeded. 51.99-styraciflua, (sweetgum) leaves palmate-lobed with the axils of the reins hairy. $h$.

\section{Order XVI. Monadelphia.}

Prncs. Staminate flowers-calyx 4-leaved : stamens many: anthers naked. Pistillate flowers -calyxes in strobiles or cones, scales 2-flowered: pistil 1 : nut with a membranaceous wing. (Perhaps more properly a samara.) h. 51. 100LEAVES EVERGREEN, 2 iN A IASCICLE.-resinosa, (yellow pine) cones not half as long as the learcsSAME, WITH 3 LEAVES IN A FASCICLE.-rigida, (pitch-pine) spines on the scales reflexedSame, witil 5 Leaves IN a FASCICle--strobus, (white pine) strobiles cylindric lunger than leaves. -Leaves DECiduous FASCICLED-hendula, (black larch) strobile oblong-microcarfia, (hacmatack) 
strobile roundisi, few-flowered.-Leaves solitaRY, SCALES OF THE CONE SMOOTH AND TAPERING. - balsamea, (balsam fir) leaves flat emarginate.canadensis, (hemlock tree) leares flat denticulate two ranked.—alba, (single spruce) leaves 4-sided incurved, strobile sub-cylindric with obovate scales. - nigra, (double spruce) leaves 4-sided strait crect, strobile ovate with oval scales.

Cupressus: Staminate flowers-ament imbricate : calyx a scale : corol 0 : anthers 4 , sessile. Pistillate flowers-ament strobilaceous : calyx a 1-flowered scale : corol 0 : stigmas 2, with concave points : nut angled. 51. 100-thyoides (white cedar) branchlets compressed and covered with imbricated rows of short leafets, cones minute and angular. $h$.

ThuJa. Staminate flowers-ament imbricate: calyx a scale : corol 0 : anthers 4 . Pistillate flowers-ament a strobile : calyx a 2 -flowered scale : corcl 0 : nut 1 , surrounded with a marginal wing. 51. 100-occidentalis (arbor vitae $\uparrow$ ) branches spreading, cones smooth with obtuse scales. $h$.

Acalyfia. Staminate flowers-calyx 3 or 4leaved : stamens 8 to 16 . Pistillate flowers-caly $\mathrm{x}$ and corol like the staminate: styles 3, 2-cleft: capsuie tricoccous, 3-celled. (Having large bracts.) 38. 95 -virginica, (three seed mercury) leaves lance oblong.

Ricixes. Staminate flowcrs-calyz 5-parted : stamens numerous. Pistiliate flowers-calyx 3 parted: styles 3, 2-cleft: capsules echinate, 3celled, 3-seeded. 38.96-communis (palma-christi, or castor oil plant) leaves palmate peltate. (-).

Phyllanthus. Staminate flowers-calyx 5 or

$\dagger$ This tree is also called white cedar in Dr. Hosack's ca. talogue ; to which and to Phelps' Calendar, we generally re. fer, as the standards for Englikh names. 
6.parted : filaments united in a column; anthers 3 and generally several barren filaments. Pistillate flowers-calyx and corol like the staminate: nectary with a 12 -angled margin: styles 3 : capsule tricoccous. 38. 96-obovata, (leaf-floner) leaves obovate obtusish.

Moxordic 4 . Staminate flowers-calyx 5-cleft : corol 5-parted: filaments 3. Pistillate flowersstyle 3-cleft: berry gourd-like and bursting elastically: seeds compressed. 34. 97-balsamina, (balsam apple) leaves cut-palmate, fruit angular. (:). e. -echinata, fruit setose-echinate, leaves heart-5lobed. (3)

Sicyos. Staminate flowers-calyx 5-toothed: corol 5-parted: filaments 3. Pistillate flowersstyles 3-cleft : berry gourd-like, 1-seeded. 34. 97 -angulata, (single-seed cucumber) leaves angular. 5. w.

Cucurbita. Staminate flowers-calyx 5-toothed: corol 5-cleft: filaments 3. Pistillate flowers - calyx and corol lilie the staminate : pistil 3 -cleft: pomaceous berry large, 3 to 5 -celled : seeds thickenerl at the margin. 34. 97-lagenaria, (sourd) leaves cordate round-obtuse hairy, fruit clavate with a woody rind. e.-verrucosa, (warted squash) leaves cordate deeply 5 -lobed with the midd!e lobe narrowed at its base, fruit clavate warty. e. - melofiefio, (flat squash) leaves corclate obtuse sub-5lobed, fruit depress-bossed. e-nchio, (pumpkin) leaves cordate obtuse sub-5-lobed, fruit roundish or oblong smooth. e. Var. 10tiro, fruit depressed or flattened-citrullus, (water-melon) leaves 5lobed, lobes sinuate-pinnatifid obtuse, fruit oval smooth. e.

Cucums. Staminate flowers-calyx 5-toothed: corol 5-parted: filaments 3. Pistillate flowerscalyx and corol like the staminate: stigmas 3 
thick, 2-parted : berry with sharpish seeds. 34. 97 -sativus, (cucumber) angles of leaves strait, fruit oblong rough. (-). y. e.melo, (musk-melon) angles of leaves round, fruit torulose. e.

Melothria. Staminate flowers-calyx 5-toothed: corol bell-form : filaments 3. Pistillate flowers-style 1: stigmas 3: berry 3-celled, manyseeded. 34. 97-hendula, (creeping cucumber) leaves 5-lobed, peduncles 1 -flowered. $y$.

\section{CLASS XXI. DIOECIA.}

\section{Order II. Diandria.}

VAllisneria. Staminate flowers-spathe 2: parted: spadix covered with florets: perianth 3 parted. Pistillate flowers-spathe 2-cleft, 1-flowered : perianth 3-parted, superior: corol 3-petalled: capsule 1-celled, many-seeded. (Stamens 2 and 6) 1.22-americana, (tape-grass) leaves linear, peduncles strait. 2\%.-sfiralis, peduncle of the fruit spiral, leaves linear with tapering base.

Salix. Staminate flowers-ament cylindric : calyx a scale, with a nectariferous gland at the base. Pistillate flowers-ament and calyx like the staminate : style 2 -cleft : capsule 1 -celled, 2 valv-

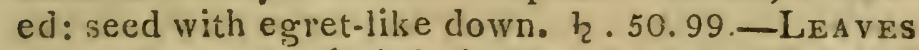
SERRATE-nigra.(brittle-joint willow) leaves lanceolate acuminate glabrous with hairy petioles, staminate flowers 4 -stamened and in perfection after the leaves appear-conifera, (cone willow) leaves lance oblong remotely serrulate glabrous above and downy beneath, stipules lunate.-eriocephala, (swamp willow) twigs downy, leaves oblong-oval sub-retuse at base, aments downy.-vitellina, (yellow willow) leaves lance-ovate acute glabrous above -lucida, leaves glabrous oblong acuminate with glandular serratures, staminate flowers in perfec- 


\section{CLASS XXI. ORDERS III AND IV.}

tion after leaves appear-babylonica, (weeping willow) leaves glabrous lance-linear, branches pendant. e.-Leaves entiRe-tristis, leaves lanceolate tapering to both ends, downy beneath-rehens, (creeping willow) stem depressed, leaves lanceoval.

Fraxinus. Ferfect flowers-calyx 0, or 4 -parted: corol 0, or 4-petalled : pistil 1: samara 1-seedcll with a lanccolate wing. Pistillate flowers on a distinct plant: calyx, corol, pistils same as staminate. $h .44 .37$-americana (white ash) leafets subovate, acuminate entire-juslandifolia, (walnut leaved ash) leafets ovate serrate.

\section{Order III. Triandria:}

Fic e's. Common receptacle fleshy enclosing the apetalous florets, either in the same or in a distinct individual. Staminate flowers-calyx 3-parted.Pistillate flowers-calyx 5-parted: pistil 1, lateral: seed 1, covered with the closed permanent somewhat fleshy calyx. 53.98-carica, (fig tree) leaves palmate about 3-lobed, fruit glabrous pear-form.

* Carex.

\section{Order IV. Tetrandria.}

Hiprophae. Staminate flowers-calyx 2-parted: corol 0. Pistillate flowers-calyx and corol like the staminate: style 1 : berry 1 -seeded. 16.24 - canadensis, (sea-buckthorn) leaves ovate.

Myrica. Staminate flowers-ament oblong : calyx an ovate scale: corol 0 . Pistillate flower's-calyx and corol like the staminate: styles 2: drupe or berry 1-seeded. 50.99-cerifera, (bay-berry) leaves lanceolate slightly toothed. h.-gale, (sweet gale) leaves lanceolate slightly serrate. $h$.

Broussonetia. Staminate flowers-ament cylindric : calyx 4-parted. Pistillate flowers-ament 
globose : calyx tubulous, 3 or 4-toothed : germ becoming fleshy, clavate, prominent: styles lateral: seed 1, covered by the calyx. 50, 99.--hafiyrifera, (paper mulberry) leaves lobate or undivided, $h_{\text {. }}$ e.

\section{Order V. Pentandria.}

Hamitosia, Perfect flowers-calyx superior 5 -cleft : $\operatorname{corol} 0:$ nectary with a 5 -toothed disk: stamens 5 : pistil 1 : fruit a drupe. Staminate flowers like the perfect, except in wanting the pistiloleifera, (American oil-nut) leaves oblong, racemes spike-like hairy. $h$.

Zixthoxrucy. Staminate flowers-calyx 5. parted: corol 0: stamens 3 to 5. Pistillate flowers-pistils 3 to 5 : capsules equal to the number of pistils, l-seeded. 46.94-fraxineum, (prickly ash, or tooth-ache tree) leaves pinnate, leafets ovate ob. scurely serrate, umbels axillary. $h$. Among a great number of these plants which I examined near Yale and Williams' Colleges, I have never been able to find a staminate flower.

Spinaera. Staminate flowers-calyx 5-parted: corol 0. Pistillate flowers-styles $4:$ seed 1 , within the indurated calyx. 12.29-oleracea, (spinach) fruit sessile, unarmed or sessile. w. e.

AcIIDA. Staminate flowers-calyx 5-parted: corol 0. Pistillate flowers-calyx 3-parted: style 0 : stigmas 3 , sessile : capsule 1-seeded. 53. 29cannabina, (water-hemp) leaves lanceolate, capsules slender acute-angled. - - ruscocartia, capsules obtuse-angled.

Humules. Staminate flowers-calyx 5-leaved: corol 0. Pistillate flowers-calyx 1-leafed, entire, oblique, spreading: styles 2 : seed 1 , within the leaf-like calyx: inflorescence strobile-form. 53.93 -lufulus, (hop) stem twining dextrorsum

Caxxabis. Staminate flowers-calyx 5-parted. 


\section{CLASS XXI. ORDERS VI, VIII TO XIII.}

Pistillate flowers-calyx 5-leaved, entire, gaping laterally: styles $2:$ nut 2 -valved, within the closed calyx. 53.98-sativa, (hemp) stem hairy, leaves digitate, staminate flowers solitary. 0 .

Nyssa. Perfect flowers-calyx superior, 5 -parted : corol 0: pistil 1: fruit a drupe. Staminate flowers-stamens 10. 12.24-biflora, (tupelo tree, or pepperidge) leaves ovate-oblong entire, peduncle of perfect flowers 2-flowered. $h$ - - triflora, (sour gum) leaves oval, peduncle about 3 -flowered. $h$.

\section{Order Vi. Hexandria.}

Smrix. Staminate flowers-calyx 5-leaved: corol 0. Pistillate flowers-calyx and corol like the staminate : styles $3:$ berry 3 -celled : seeds 2. 11. 12-rotundifulia, (green briar) stem prickly, leaves cordate acuminate 5 to 7 -nerved, climbing. $h \cdot w-y-$ heduncularis, (Jacob's ladder) stem terete unarmed: leaves ovate acuminate 9-nerved, peduncles of fertile umbels longer than the leaves. 24. g. -herbacea, stem and leaves unarmed, leaves 7 -nerved, climbing with tendrils. 4 . w-y. - sy hhilitica, (true sar'saparilla) stem prickly-terete, leaves lance-oblong 3-nerved mucronate.

Droscon corol 0. Pistillate flowers-styles 3: capsule 3celled, compressed : seed 2, membranaceous. (Leaves generally alternate.) 11.12-villosa, (yam root) leaves downy beneath, cordate. 2\%.

From Order Vili. Octandria, to Order XiII. Polyandria.

Populus: Staminate flowers-ament cylindric: calyx a torn scale : corol turbinate, oblique, entire. Pistillate flower's-ament, caly $\mathrm{x}$ and corol like the staminate : stigma 4-cleft : capsule 2-celled: sced 
with egret-like hairs. (Leares having a tremulous motion.) h. 50. 93-tremuloides, (white poplar) leaves heart-roundish abruptly accuminate serulate-toothed hairy edges-trenida, (high poplar) leaves round-oval smooth both sides, unequally sinnate-toothed. (Young leaves downy.)-angulata, (bahn of gilead) leaves cordate obtusely hook-toothed glabrous, branches with winged angles-balsamifera, (balsam poplar) leaves ovate close serrate whitish or rusty beneath, stipules gummy, buds resinous-dilatata, (lombardy poplar) leaves deltoid. e.

Diospyros. Staminate flowers-calyx 4 to 6 cleft dilated : coral pitcher-form, 4 to 6 cleft : stamens 8,16 or 29. Pistillate flowers-calyx and corol like the staminate: styles 4 -cleft : berry 8 to 12 seeded. 1841 --virginiana, (date plumb) leaves ovate obtusish glabrous. $2 \%$. w.

Gleditschia. Perfect flowers-calyx 4-cleft : corol 3-petalled: pistil 1: capsule legume-like. Staminate flowers-calyx 3-leaved; corol 3-petalled. Pistillate flowers-calyx 5-leaved : corol 5 petalled. 33. 93-triacanthos, (honey-locust) leafets 10-paired, leaves doubly-pinnate. $h$.w.

DATISCA. Staminate flowers-calyx 5 leaved: corol 0 : anthers sessile, about 15 . Pistillate flowers-calyx superior 2-toothed: styles 3 : capsules 3-angled, 3-horned 1-celled, pervious. 54.-hirta, (false hemp) leaves pinnate, stem hirsute. $\boldsymbol{\psi}$.

Mexispermum. Staminate flowers-calyx 2leaved : petals 4 or 6 outer and 8 inner: stamens 16. Pistillate flowers-corol like the staminate : 8 barren stamens: germs 2 or 3 : berries paired, 1 seeded. 11.77-canadense, (moonseed) stem twining, leaves peltate cordate round angular. $h$.w. --virginicumi, leaves sub-peltate cordate lobed pubescent beneath $h w$. 


\section{Order XVI. Monadelphia.}

JUNIPERUS. Staminate flowers--ament ovate : calyx a scale: stamens 3. Pistillate flowers-ca. lyx 3-parted: petals 3 : styles 3 : berry 3 -seeded, consisting of the three unequal tubercles of the calyx. (Nut bony, 1-celled with balsamy glands at the base.) 51.100--virginiana, (red cedar,) leaves united by their bases in threes. $h$-communis, (juniper) leaves ternate (or three ranked) spreading mucronate longer than the berry--sabina, (savin) leaves opposite obtuse imbricate 4-ways. $h$.

Taxus. Staminate flowers--calyx about 4-budleaves : corol 0: stamens many, anthers peltate, 8cleft. Pistillate flowers--style 0 : receptacle acorncupform; nut ovate: 51.100-canadensis, (yew) leaves linear pointing 2 -ways, margin revolute. $2 \%$.

\section{Polygamous Plants.}

Some authors still retain the class of Polygamia; but we, following Persoon, have distributed the genera belonging to this class as follows :-Androtzogon to class 3 , order 2. Holcus to 3, 2. Panax to 5, 2. Atriflex to 5,2. Celtis to 5,2. Veratrum to 6, 3. Melanthium to 6, 3. Acer to 8, 1. Mimosa to $16,10$. Parietaria to 20, 4. Fraxinus to 21 , 2. Nyssa to 21, 5. Gleditschia to 21, 13.

\section{CLASS XXII. CRYPTOGAMIA.}

\section{Order I. Filices.}

Ferns are annulated, when each capsule has a kind of chain passing around it at about right angles with the suture. When the fruit is ripe the chain in straitening opens the capsule; it being attached by its ends to two contiguous edges of it. 


\section{A. With annulated caflsules.}

\section{Without involucres.}

Acrosticher. Capsules numerous, covering the whole lower surface of the frond. (The fertile leaves differ in shape from the barren. This genus should be examined in the young state ; for the scattered fruit of other genera, by becoming confluent when old, often cover the frond.) 55.5-aureum (fork-fern) frond pinnate with alternate leafets, fruit on the upper leafets $2 \%$.

PoLfPodicy. Capsules disposed in round scattered clots, on various parts of the lower surface of the frond. 55. 5-vulsare, (polypod) frond pinnatifid with oblong obtuse sub-serrate lobes, root with chaffy scales-incanum, same, with lobes lanced entire-virginianum, root smooth-connectile, frond doubly-pinnate, ciliate, fruit-dots minute-calcare. wm, frond ternate and doubly-pinnate.

\section{With involucres.}

OxOCLEA. Fruit-dots incleterminate, capsules covering the whole lower surface of the frond: involucre formed by turning in or rolling back the margin of the leaf, which opens inwards, in maturity, towards the midrib. (The fertile leares are contracted and narrower tian the barren ones.) 55. 5-sensibilis, (sensitive polypod) barren frond pinnate, fertile one doubly-pinnate-_struthiofteris, fronds doubly-pinnatifid, divisions roundish.

Blechxis. Capsules in uninterrupted lines running parallel to the midrib of the frond on both sides : involucre opens inwards.t (The fruit of

$\dagger$ The involucre opens inwards, when it opens or sepa. rates, so as to leare the capsulcs naked on the side next to the midrib. 
this genus, when ripe, often covers the whole lower surface of the frond like the acrostichum; it must therefore be examined in the young state.) 55. $5-$ borealis, (Roman fer $n$ ) barren frond pinnatifid, fer. tile one pinnate with acuminate leafets-serrula. tum, frond pinnate, leafets serrate tapering to both ends.

Pteris. Capsules arranged in a continued line along the very margin of the frond : involucre opening inwards. (When the leaves are extremely small, the rows of capsules on opposite sides meet and cover the lower surface like the onoclea.) 55. 5aquilina, (common brake) leafets pinnate with lanceolate repand-tocthed divisions, upper ones smallest-fracilis, leafets lanceolate obtuse alternate, lower ones pinnatiff-caudata, leafets coubly-pinnate, subdivisions sub-linear-atrofurfurea, frond doubly-pinnate, subdivisions sub-linear, lines of the capsulcs somewhat interrupted.

Vitraria. Capsules in an uninterrupted line along the disk or margin of the frond: involucre double, one of which opens inwards, the other cutwards. (Leaves always simple.) 55.5-lineaic, (ribbon fern) frond linear very long pendant.

Asplexium. Capsules in lines parallel to each other, situated exactly uffon the secondary veins of the frond: involucres opening invards. (By the secondary veins is meant those of the middle part of the disk, not the larger ones at the margin or midrib. The parallel lines of capsules may stand obliquely with respect to the midrib, but their direction must be parallel to each other.) 55.5FroNd DOUBLY-PINNATE BELOW.-montanum, (spleen wort) leafets pinnatifid-ruta-muraria, frond only pinnate at top with obtuse leafets.-Frond PINNATE ONLY-thelyhteroides, leafets deeply-pirnatifid with tooth-serrate divisions-angustifolizm, 
base of upper leafets truncate, of lower ones rounded-ebeneum, leafets serrulate, upper ones eared, lower ones cordate-melanocaulon, leafets cienate with wedge-form base. FroND SIMPLE一 Thyzofihyllum, (walking-leaf) frond lanceolate with a rooting apex.

Scolopexdrum. Capsules in strait or zigzag parallel lines between the secondary veins (or ribs) of the frond: involucre clouble, opening on the top of the lines of capsules and folding down towards each side. (In the genus csfleniam, the lines of capsules lie across the veins of the frond; but in this they are in line with the reins and attached along their sides or between them.)-vulgare, (caterpiliar fern) capsules between 2 veins.

WOODWARDIA. Capsules in oblong or oval spots arranged in regular rows on both sides of the midrib : involucre vaulted and opening inwards. (When the leaves have large segments, the capsules are arranged along the midribs of the seg. ments also.) 35.5-angustifolia, (kidney-fruit fern) frond pinnate, segments linear acute entire, barren ones sub-serrate-virginica, segments pinnatifid.

Adiaxtum. Capsules disposed in oblong spots arranged along the margin of the frond : involucre is formed by turning back the margin of the frond over the capsules, and it opens inwards. (The lines of oblong spots are generally along that margin, which may be considered the end of the leaf or of the segments of the leaf.) 55. 5-hedatum, (maiden-hair) frond pedate, branches pinnate, leafets halved.

Aspidium. Capsules in scattered roundish spots on various parts of the whole lower surface of the frond: involucie a kidney-form or round membrane, fastened to the frond in or near the center of the fruit-dot, and opening on all sides. (The 
involucre, when a little opened, is strictly pellate.) 55. 5. FROND TERNATE-crucitarium, (shieldfern) leafets pinnatifid.-FroND PINNATE WITH UNDIVIDED LEAFETS- acrostichoides, leafets ciliateserrate. Frond PINATE With rinNatifid LEAFETS-noveboracensis, divisions of leafets sub. entire ciliate obtusish-cristatum, divisions of leafets tooth-serrate obtuse. Frond Doubly-pinvate. -felixmas, leafets serrate. Var. erosum, leafets erose-serratc-marginale, leafets crenate decurrent. Aтrynium. (Taken from asficidium.) Capsules in scattered roundish or oblong spots on various parts of the whole lower surface of the frond: involucre a roundish membrane fastened to the frond at one side of the fruit-dot and opening on the other. (The involucre is generally fastened inwards and opens outwards towards the margin of the frond.) 55. 5. FroNd PINNATE-thelypteris, (snuff-box fern) divisions of leafets acute. Frond DOUBLY-PINNATE, LEAFETS DOUBLY-SERRATE- $f e$ lix-femina, fruit-dots strait-oblong-ashlenoides, fruit-dots lunulate. SAME, WITH LEAFETS SERRATE-bulbiferum, leafets opposite, fruit-dots round. SAME, WITI LEAFETS NOTCHED OR TOOTHED-tenue, leafets decurrent, toothed-rufidulum, leafets crenate with united bases, chaff-bristly beneath.

Dicrsonia. Capsules in small round scattered fruit-dots at the margin of the frond: involucre double; one part is formed of the thin margin of the frond turned over upon the fruit-dots, the other is from the frond inward side of the fruit-dots, each part opening opposite to where it is fastened to the frond. 55.5-pilosizuscula, (fine-haired fern) frond cloubly-pinnate, leafets pinnatifid.

Woodsis. Involucre perianth-form, with a hairy margin: capsules pedicelled included within the 
involucre : fruit-clots roundish, scattered. 55.5hyfierbolea, (calyxed ferri) frond pinnate, leafets 3 parted-ilvensis, frond doubly-pinnatifid, divisions oblong.

Cheilanthes. Fruit-dots roundish, distinct, situated at the margin of the frond: involucre a roundish membrane formed by turning over the margin of the frond upon the fruit dots, and opening inwards. (The margin is always crenate, and each fruit-dot has its own involucre perfectly separate from the others.) 55.5-vestita, (lip-fern) frond doubly-pinnate with pinnatifid leafets, hairy both sides.

\section{B. With cahsules not annulated.}

Schizaea. Capsules top-form, striate longitr: dinally and radiately, situated upon the back part of a narrow spike-form appendage of the frond, surrounded with or interspersed among, bundles of hairs. (The appendages, bearing the capsules are generally digitate and occupy the summit of the frond.) 55. 5-tortuosa, (one-sided fern) frond simple, spikes one-way.

Osuunda. Capsules giobose, pedicelled, radiate-striate or wrinkled, having a hinge at the joining of the two valves, which resembles part of the jointed ring of annulated ferns : the capsules either occupy the whole lower surface of the frond, or a panicled-raceme. (The parts of the frond occupied by the fruit are always more contracted than the barren parts.) 55.5-cinnamomea, (flowering fern) frond doubly-pinnatifid, fertile ones distinct and panicle-racemed-interrufita, barren leafets on the frond with the fertile ones, above and below and sometimes among them-inectabilis, frond doublypinnate, leafets sharp-serrate-regalis, frond bipinnate, terminating in several racemes, very branch. ing and without hairs. 
LyGodrum. Capsules radiate-striated or wrinkled, opening laterally, longitudinally or transversely; they are situated upon spikelets which proceed from the margin of the frond, each capsule being hid under a scale which falls off frequently in ripening. 55. 5-falmotum, (fringed fern) leaves palmate.

\section{APPENDIX TO THE ORDER FILICES.}

Prerotdes.

Plants related to the ferris in their general ha. bit; but in some degree approaching the habit of pines, umbelliferous and culminiferous plants. The young leaves are never spiral ; in which particular they differ from most of the proper ferns.

Botruchium. Capsules coriaceous, globose, one-celled, smooth; they are disposed in spikes or racemes, generally open lengthwise, sometimes irregularly. 55. 5-FROND 3-PARTED, BIPINNATIIID-gracile, (grape-fern) divisions of the leafets sub-pinnatifid and sub-clentate-dissectum, divisions 2-parted, 2-toothed at the apex-virginicum, divisions obtuse, sub-3.toothed.- CAME, BIPINNATEfumaroides, leafets lunate crenate- DOMEWHAT DOUBLY.TERNATE-obliquum, leafets serrulate, unequally cordate.

Ophioglossum. Capsules round, 1-celled, opening transversely; they are placed on a somewhat jointed spike in two close rows. 55.5-vulgatum, (adder-tongue furi) frond ovate simple, spikes about an inch long.

Psilotum. Capsules globose, 3-celled, 3-seeded, opening upwards with three pores; they are sessile on the branches and axillary. 55.5-dicho. toma, (naked fern) stem naked 3-sided forked.

L. ICOPODIUM. Capsules mostly kidney-form or 
roundish, 2 or 4-valved opening elastically; they are placed under separate scales in a spike, or sometimes in the axils of leaves. (Very leafy, their stems being generally covered with 2,3 or 4 rows of narrow simple entire leaves.) 55.5-comhlanatum, (club-moss, or ground pine) leaves acute 2-rowed united, spikes in pairs or in fours peduncled erect, stem creeping-obscurum, leaves scattered 6-rowed, shoots erect, branches radiately forked, spikes nearly sessile cylindric solitary terminal-rufestre, leaves scattered ending in a fila. ment, spikes 4-sided sessile.

Equisetum. Fruit placed under peltate bodies, which are arranged in whorls forming a spike-form raceme; four spiral filaments surround the seed (probably) which resemble green globules. (Fertile plants mostly leafless, the stems of all are jointed with toothed sheaths at every joint, and usually longitudinally striated and hollow.) 55.5hyemale, (scouring rtish) stem naked very rough, sometimes branched at the base, spike terminalarvense, (field horsetaii) fertile stem simple naked, sheaths broad sharp black distinct-sylvaticum, (woods horsetail) fertile stem with a few whorls of branches, teeth at the joints broad-pointed.

\section{Order II. Muscr. $\dagger$}

\section{A. Caflsules without a lid.}

Prescum. Capsule furnished with calyptre, but

t So far the most common species of each genus have been given. Perceiving that the work is swelling beyond what was intended; nothing more will be given under the five remaining orders, than is absolutely necessary for studying the genera. Perhaps another edition may appear, which will include all the known species of plants in the Northern States. The manuscript was prepared last summer, under the immediate inspection of Professor Ives of Yale College; and it is now ascertained, that it would not extend this book beyond another bundred pages. 
having no lid it falls off closed. (The smallest of mosses, never half an inch high, arid more commonly not the eighth of an inch, and almost sessile.) 56. 4-crisfum, (earth moss) having a stem, learcs lance-acuminate.

B. Capsules ruith a lid and rvithout a freristome.

Sphignty. Calyptre breaks off transversely, leaving the lower half on the capsule: lid caducous. (Found only in swamps. Becomes reldish when old.) -latifolium, (peat-moss) leaves ovate obtuse close-imbricate.

Grmxostoxex. Calyptre wholly falling off: lid caducous-ilyriforme, (toothless moss) leaves ovate acute spread, capsule pear-form, lid obtuse pointed.

C. Cafsule with a lid and furnished with a single fieristome, frroceeding from the outer surface of the cafsule, or from both inner and outer.

\section{Teeth fewer than 16.}

TETraphis. Tecth 4 , taking their origin from both the inner and outer surface of the capsule. 56. 1-hellucida, (four-toothed moss) nearly transparent in various parts.

Splachum. Teeth 8 : capsule with an umbrella-form apophysis below its base. 56. 4-amfiullaceum, (umbrella moss) apophysis blubberlike.

2. Teeth always 16, and neither shlit, connected, nor in fair?.

Grimma. Calyptre smallish: peduncle terminal. 56. 4-controversa, (awl-cap moss) leaves lance-linear, serrate at top-alficola, leaves obtus. ish, capsule obconic nearly sessile. 
Maschalocarfus Calyptre small : peduncle arillary : stem sarmentose. 56. 4-julaceum, (creeping moss) ascending, leaves ovate acute nearly as long as the peduncle.

3. Teeth alivays 16 , and sflit.

Dicranum. Each tooth split part of the way from the tip towards the base, incurved and solid. (Muhlenberg found $2 j$ species in North America.) 56. 4-bryoides, (fork moss) leaves 2-rowed com. pressed, capsule terminal erect.

Trichostomum. Each tooth split quite down to the base, erect and filiform. 55. 4-pallidum, (hair-mouth moss) stem simple short, lcaves capillary and sheathing.

\section{Tecth 16, 32 or 64 , connected.}

Polytrichum. Tips of the teeth all meet in a flat membrane, which extends over most of the mouth of the capsule : calyptre mostly composed of hair. 56, 4-junifterinum, (hair-cap moss) capsule square, leaves lance-linear with rough keels.

\section{Teeth 16, 32, or 64, in hairs.}

Didysodon. Capsule without any apophysis below its base. (The sflachnum, having teeth sometimes in pairs, is best distinguished from this genus by its apophysis.) 56. 4-lineare, (doubletooth moss) leaves linear.

D. Cafisule with a lid, and furnished with a single heristome piroceeding only from the inner surface of the cafssule.

Barbula. Peristome consists of twisted filaments. 56. 4-aficulata, (spiral-tooth moss) stem short, leaves remotish lance-oblong.

Diphascum. Teeth vein-like, lying on a conic plaited membrane; capsule inflated almost sessile 
upon the root. 56. 4-foliosum, (bladder mouth) leaves linear acuminate:

E. Caflsules with a lid, and furnished rvith a double heristome, the outer one consisting of 8 or 16 distinct teeth.

1. Inner feristome consisting of teeth or flaments not connected at their bases.

Orthotrichum. Inner peristome consists of 8 or 16 fine hair-like filaments, vaulted inwards. 56 . 1-anomalum, (curve-haired moss) branched, leaves lanceolate keeled obtusish, calyptre hairy toothed base-striatum, leaves acute keeled spread, calyptre entire.

Neckera. Outer peristome consists of 16 teeth, the inner one of 16 filaments, erect; peduncles axillary. (Sarmentose.) 56. 4-finnata, (straithaired moss) decumbent branched, leaves 2-rowed, capsule immersed in the sheath-macropoda, peduncles long.

Trmmra. Outer teeth very short; inner ones 16 , long and membranaceous, in distinct pairs : peduncles terminal. 56. 4-holystachoides, (manyspiked moss) leaves lance-linear serrate spread, lid depressed in the centre.

Cirmacrum: Inner peristome consists of 16 filaments, disposed in pairs; each pair is united at top and connected below by transverse fibres: peduncles axillary. 56. 4-dendroides, (tree moss) a projecting collar below the outer teeth.

2. Inner heristome consisting of teeth or flaments connected towards the base by a membrane.

Meesia. Inner peristome consisting of 16 reticulate flat teeth: peduncle terminal. 56.4-longiseta, (net.tooth moss) having a very long awn.

Leskea. Outer teeth long-acuminate; inner 
peristome consists of a delicate membrane at the base, and upwards becoming uniform acuminate teeth : peduncle axillary. (Greatly resembles Hypnum, but the ciliae between the inner teeth of the hynum will distinguish it, if a very high magnifier be used.) - rostrata, (goose-neck moss) having a long beak. (Muhlenberg found 16 species.)

BARTRAMIA. Inner peristome consists of a delicate membrane terminating in 16 acuminately divided teeth disposed in pairs : peduncles terminal, except halleriana. 56. 4-arcuata, (bow-moss) branched, leaves serrate striate. (Muhlenberg found 5 species.)

Mnium. Inner peristome consists of a membrane terminating in pointed teeth which are much perforated with large holes compared with the size of the teeth, having between them fine hairs : peduncles terminal. 56. 4-cusfidatum, (prickedtooth moss) holes through the teeth smallish. (Muhlenberg found 11 species.)

BRYUM. Inner peristome consists of a membrane terminating in pointed teeth not perforated, having between them very fine delicate hairs : peduncles terminal. 56. 4-concideum, (thread moss) leaves entire spread, capsules erect pearformmutans, leaves serrate at top, capsule pendant. (About 12 species have been found in North America.)

HYPNU. Inner peristome consists of a membrane terminating in pointed teeth not perforated, having between them very fine hairs, to be seen with a good magnifier: peduncles axillary. 56. 4 - serfiens, (sleeping moss) creeping, leaves !-nerved at bottom, entire. (Muhlenberg examined 38 species in North America.) 
3. Inner heristome consisting of a membrane, or of united filaments.

Fontralis. Inner peristome consisting of a reticulate membrane with longitudinal fibres. 56 . 4 -antehyretica, (water moss) leaves of the sheath obtuse, stem branched-cafillacer, leaves acute, peduncle long.

Buxbauma. Outer teeth extremely short and truncate; inner peristome consists of 2 or more cylinders of filaments slightly connected together, the inner cylinder being the longest: capsule oblique : peduncle radical. 56.4-ahhylla, (leafless moss) capsule peduncled.

F. Cafsule with a lid, and furnished with a double heristome, outer one consisting of 16 connected teeth.

Funaria. Outer teeth oblique and connected at the tips: inner are 16 filaments lying flat : peduncles terminal. 56.4-hygrometrica, (rope moss) leaves inflected, peduncle becomes twisted by drying.

\section{Order 1II. Hepatrcae.}

JUNGermanxis. Capsules 4-valved with chain: like seed-dispersers, peduncled, arising from a bell-form calyx. (An apophysis adjoins the capsule, and it has a silvery filamentous peduncle, which often grows several inches in one night. The peduncle rises from a bell-form calyx, which is more permanent than the fruit. In this calyx the fruit lies concealed a while, at length it opens and discloses a small shining black globule, which rises on the silvery peduncle and bursts into 4

t The plants of this order have the fructification much like that of Mosses; but the frond or leafy part sometimes resembles that of Lichens. 
valves.) 57. 3-7rorella, (false moss) branches flowering in the middle, flowers sub-sessile, leaves obovate. (Muhlenberg found 27 species.)

Marchantia. Capsules peduncled, bursting at their summits : seeds attached to elastic fibres: the capsules are placed beneath umbrella-like stars or cones. (It agrees with some species of jungermannia in external appearance, but it is of a much firmer texture. The frond is always leafy, lobed, furnished with a strong midrib and beset beneath with villous roots. 'The disk of the leaf consists of a beautiful green network.) 57. 3-polymortika, (brook liverwort) frond obtusely lobed. (In the spring some of this species send up ovate anthers or buds on pellucid filaments from the disk of the frond. Near the middle of the summer the umbrellas appear, bearing the fruit under the rays.)

Axthoceros. Capsules horn-like, issuing from a calyx with toothed reflexed margin, covered at first with a brown villous caducous calyptre; afterwards splitting into valves and presenting the columella with the seeds attached: around the ca. lyx, within the substance of the leaf, and in a proper involucre, are inclosed heaps of reddish minute anthers or buds. (The substance of the frond is made up of beautiful 6 -sided cells, each of which is marked on the surface with a small protuberance.) 57. 3-levis, (was liverwort) frond flat, crenate-frunctatus, frond sinuate-lobed obtuse.

Riccia. Capsules sessile, globular, 1-celled, crowned with an appearance of withered anther's; they are lodged in the substance of the leaf, and to be discorered only by the darker colour of the place where situated. (Usually found with Lemna in ponds and stagnant waiers.) 57. 3-fuiluns, (gaping-liverwort) fronds repeatediy forked, linear, M 2 
smooth, reticulate obtuse, cleft at tips-natans; frond obcordate flat with long compressed radicles.

\section{Order IV. Algae.}

Fucus. Germs imbedded in the gelatinous sub. stance of the frond, aggregate : seeds produced in clustered tubercles, which burst at their summits. 57. 2-edulis, (dying seaweed) frond a roundish short stem expanding into a wedge obovate obtuse succulent leaf. An excellent vermifuge. (See Doct. A. R. Griffin's inaugural dissertation, before the New. York college of physicians.)

ULYA. Frond membranaceous or gelatinous : fruit in semi-transparent vêsicular membranes within the substance or under the general cuticle of the frond, either solitary or clustered. 57.2furrirurea, (seaweed) lance-oblong, flat, ma!'gin wary entire.

Coxferva. Consisting of herbaceous tubes $\mathrm{Cl}^{\circ}$ fibres, generally separated within by transverse partitions: seeds scattered through the joints, or contained in solitary closed tubercles attached to the tubes or fibres. 57. 2-nivida, (green-hair, frog's spittle) filaments simple lubricous shining, joints cylindric-fluviatilis, (brook grecn-hair) filaments branched alternately.

\section{Order V. Lichenes. $\dagger$}

\section{A. Recefiacle none; fropago naked, scattered or agsregate.}

I.EPRARIA. Eftlorescence sub-globular, scattered loosely over a crustaceous base, which it often

$T$ This order is wholly copied from the system of AchariIs, translated by Doct. Smith. Acharius has since subdivid. ed Parmelia, Baemyces, \&c. by giving generic names to the sections of species. Sprengel says, these three first grencra are merely the first rudiunsonts of o:ber genera, and should be rejected. 
cover entirely : frond crustaceous, indeterminate. 57. 2-chlorina, (leprous-like lichen*) greenish yellow or dark deep yellow, thick, effuse.

Spiloma. Efflorescence in shapeless superficial masses, woolly and powdery, rigicl : frond crustaceous mostly indeterminate, but sometimes border$\mathrm{ed}$, thin and uniform. 57.2-melanofra, (efflorescent lichen) blackish flat sub-confluent.

B. Recentaclest composed of a heculiar compact hardish substance, not in any manner formed out of the frond, from which it differs in colour, being often black.

\section{Recentacle onen.}

VARTOLARia, Receptacles seated on a thicis tartarous crust, which supplies them with an accessory border; thcir disk is occupied with an assemblage of powdery substance : the frond is generalby determinate. This genus was supposed to have ro receptacle, until Dr. Smith detected the error. 57. \&-faginea, (small-pos lichen) frond leprous thin glabrous grey, receptacles white.

Opegrapha. Clefts black, sessile, oblong or linear, simple, confluent or branched, strait or zig-

* Young botanists who have not been informed to what department of nature the word lichen applies, may be told, that the following substances are licliens. The spots on rocks and stones which are white, yellow, black, \&c. sprinkled over with dots resembling fly-dirt upon window-glass. Also the whitish green patches on old fences, trees, \&c. and the substance on trees of a fibrous structure and firm tezture, usually called moss. It differs from moss in being more tough and hard, and generally less green.

$t$ is the student will find nothing on lichens like those organs denominated the fructification of phenogamous plants, he may be told that by receptacles are here meant those specks or dots mentioned in the last not-, or those sutucerform substances on the large greenish patches on fences. 
zag, bordered : their disk flat or slightly concave, naked or powdery, narrow, linear, rarely dilated: their border is of their own substance, often very narrow and scarcely perceptible, sometimes tumid, elevated, and sometimes inflexed : frond crustaceous, uniform, thin, generally indeterminate, occasionally leprous. 57.2-scrifita, (lettered lichen) clefts zigzag.

LECIDEA. Spangles sessile, with a flattish or convex disk : frond various, crustaceous; uniform and limited, scattered and indeterminate, leafy, membranous, or stellate. 57.2-immersa, (spangled lichen) greenish white, spangles black.

Calicium. Puffs turbinate, lenticular, or nearly globose : generally elevated on a rigid simple stalk of a different substance from the crust : frond crustaceous, leprous or tartarous, uniform or scattered, powdery and granulated or solid and chequered, or somewhat leafy and imbricated. This genus was confounded with the fungi, till distinguished by Persoon. 57.2-strigonelium, (fungus lichen) crust none.

2. Receftacles closed, at length ohening.

Gyrophora. Buttons superficial flattish: frond leafy, peltate, between membranous and leafy. 57 . 2-vcllea, (button lichen) brownish grey, peltate.

Bathelium. Receptacles sessile, sub-globose, opening by a decirluous lid, hollow, from 1 to 3 cells lined with seeds : frond crustaceous, inde. terminate, uniform.

VERRUCARIA. Tubercles elevated, discharging their chained seeds by a terminal pore, or else they fall out entire: frond thin crustaceous, uniform. Very minute. 57.2-nicidla, (mite lichen) pale sleen, tubercles crowded.

Exdocarpon. Hollows hidden in the substance 


\section{CLASS XXII. ORDER V.}

of the frond, each opening by a little mouth : seeds enclosed in cells: frond membranous, somewhat crustaceous, of a determinate figure; so united to the substance of the receptacles as to appear of a piece, excepting a little difference of colour. 57. 2-miniatum, (hidden lichen) peltate, pale, with spread-lobes.

C. Receptacles formed of the frond itself, with wohich it agrees in substance and colour: seeds (excepting of the sphaerophoron) enclosed in firoher cells.

1. Recefulacles comfround, either hierced or safing. Thelotrema, Receptacles compound; the outer ones wart-like, elerated, of the substance of the crust ; inner ones somewhat globular, with a concave disk : frond a firm, continued, indeterminate crust. 57.2-hertusus, (crust lichen) warts hemispheric with 1 or 2 pores.

2. Recefutacles nearly globular, closed; at length bursting.

Sphaerophoron. Cellules nearly globose, each enclosing a roundish ball of naked compact seeds, which turns to black powder, and is discharged by the torn orifice, leaving the cellule hollow and empty : frond shrubby, branched, solid, rigid, tartarous or minutely fibrous within; with a smooth cartilaginous coat. A very elegant genus resem. bling corol. 57.2-slobiferus, (seed-ball lichen) shrubby branched cylindric brownish smooth.

IsIDIU s. Receptacle of the seeds terminal, subdiscoicl, solid, various in thickness, covered with, and shining through, the crust of the papillae of the frond, which at length bursts, and each recepta. cle appears coloured and partly exposed : globules also either terminate each papilla or are nearly ses- 
sile on the crust itself; which are coloured and finally fall out, leaving pits at the summits of the branches : frond crustaceous, leprous and tartarous, cracked, rather indeterminate ; either papillary only, or throwing up solid branches. Resembles sphaerophoron; but differs in having a base, terminal globules, \&c. 57. 2-corallinus, (coral lichen) warty-branched, tartarous, grey.

3. Receftacles orbicular, ofien; their border of the colour of the frond, surrounding a variously-coloured disk.

URCEOLARIA. Shields or spangles cup-form, variously coloured, sunk into the substance of the crust, or of its fragments or warts : disk concave, rarely becoming flattish by age, but never elevated above the crust: proper border often scarcely manifest, of the colour of the disk ; the accessory border sometimes formed of the crust, elevated like a ring surrounding the disk of each shield : frond. crustaceous, tartarous, uniform, determinate, chequered or cracked, rarely figured or lobate. Often found on stones, \&xc. and some species are inseparable from the stones on which thicy grow. 57.2bryothilla, (pitcher-shield lichen) greyish with glaucous-black shields.

Parmelia. Shields superficial or elevated, thick or sub-membranous, flattish, convex, or concave, crowned with a free accessory border : frond various, crustaceous, leafy, branched or laciniated, cartilaginous, membranous or gelatinous. A vast genus. One of its best characters is, that the shield has no border of its own substance; but always has an accessary one of the substance of the crust. 57. 2-herbacea, (shield lichen) bright green with fulvous shields. 
4. Receftacles fattened, nearly destitute of a bor. der; their ufither side entively covered with a thin seed-bearing disk, of considerable solidity at the surface.

Sticta. Shields on the upper side of the frond, sessile, close-pressed, orbicular, membranaceous; their disk flat, rarely somewhat convex; their border accessory, thin, entire, free, slightly. tumid, rarely fringed, of the colour of the frond : pits among the down of the under side, scattered, sessile, minute, coloured: their disk roundish powdery; border elevated, a little inflated, entire or jagged, sometimes obliterated : frond leafy, membranous, or in some measure leathery, lobed or jagged, downy or shaggy beneath. The pits on the back of the frond form the best of its characters. 57. 2-sylvatica, (pit-back lichen) glaucous. brown, shields marginal brown.

Peltidea. Targets closely attached to the frond about the margin on the upper or under side, rarely lateral or towards the middle, each consisting entirely of a flattish (rarely concave) coloured disk : border accessory, very thin and scarcely discernible, united with the frond and of the same colour : frond rather leathery, with downy veins and fibres beneath, rarely naked; the circumference lobed, its barren lobes depressed, fertile ones elongated, ascending, naked at the base. Best distinguished from the sticta by having prominent veins or ribs underneath, and no pits. 57.2-venosa, (target lichen) grey with divergent lobes, targets round brown.

CETRARIA, targets shield-like, thickish, flat, close-pressed, sessile, near the margin; their circumference loose, rounded, deformed or wavy, disk coloured, slightly convex : border accessory, 
very narrow, entire or crerate, of the colour of the frond: frond leafy, membranous or sub-cartilaginous, rigid, smooth and naked on both sides; sometimes cellular or pitted; lobed and jagged, often crisped at the edges. The receptacles are rather between shields and targets; well distinguished from Pelridea by its smooth, poiished, membranuus, sub-rigid frond. 57.2-isiandica, (iceland lichen) chesnut-greenish, ciliate-prickly.

Cormetraria. Urbs shie!d-like, terminal, peltate, thickish, cartilaginous, orbiculor, flat; at length rather conrex, uneren, irresular ; disk generally of the colour of the frond; border scarcely iny, except in a young state a straight accessory one, entire, naked, or radiated, at length reflexed: frond cartilaginous, rigid, smooth, rather solid, spongy within, of a shrubby tufted branched habit, the branches acute. Resembies the Palmelia in the frond and the Usnea in the fruit. 57. 2-tristis, (horned lichen) brown, branches cylindric-muricata, horns lateral and terminal.

UsveA. Orbs sub-coriaceous, flattened, peltate, naked and smooth on bo:h sides, mostiy much dilated; disk first concave, then flat, even, subsequently rather convex and cracked, or warty, scarcely coloured; border either none or accesso$\mathrm{r} y$, which is entire or toothed in the circumference, very often radiated: knobs on the same, or on a different plant, which are sessile, lateral, scattered; at first shield-like and sometimes in a manner bordered, finally convex and warty, coloured. This is the filamentous lichen, tapering, more or less jointed, having a central elastic medullary thread. It is sometimes called tree-moss. 57.2-fllicata, (filamentous lichen, or tree-moss) pendant, palish white, orbs greenish white. 
5. Recefitacles convex, more or less globose, clothed with a seed-bearing layer, or disk, sessile on the branches, patillary firocesses, or pieculiar stalks of the frond; terminal and hermanent.

Stereocaulon. Knobs sub-turbinate, at first furnished with a proper border, then globose, without stalks, simple or clustered, coloured, permanent; disk at first flat and small, but gradually occupying the whole; the border of the same uninterrupted substance and colour, entire, at length obliterated : frond solid, almost woody, caulescent, erect, branched, mostly clothed with scales, rough and fibrous. 57. 2-ramulosus, (knobbed lichen) fibrous rough shrubby scale, knobs dark brown.

BAEMTCES. Knobs capitate, nearly globose, swith reflexed and scarcely bordered edges, terminating their own proper fruit-stalks, simple or clustered, coloured, permanent; disk properly none, the whole globose surface being covered with the thin, solid, seed-bearing coat; border none : frond either subcrustaceous, softish, granulated, indeterminate, rarely figured ; or consisting of a cartilaginous leafy and lobed crust : fruitstalks eloneated out of the very substance of the frond, simple or branched, tubular or nearly solid, dilated or tapering upwards, sometimes barren. 57. 2-cocciferus, (scarlet-mouth lichen) stem and cup greenish grey, knobs scarlet, sessile on the edges of the cup.

\section{Order Vi. Fungi.}

Agaricus. Fungus with gills underneath. 58 1-camfiestris, (eatable mushroom) gills pirk-colour, stem white, with volva.

Merulies. Fungus with veins underneath. 58. 1-cnrnucoxoides, (veined fungus) stem groved, pileus dull-yellow. 
Boletus. Fungus with pores underneath. 58. 1-igniarius, (touch-wood) form of a horse's hoof.

THAELAEPHORA. Fungus warty underneath, the substance corky. 58. 1-caryoflhyllca, (pink fungus) brown with radiate lines and white edge.

HyDNum. Fungus prickly underneath. 58. 1chrysorhizum, (paper punk) membranaceous flat spreading stemless, root yellow filiform, extending along the grains of decaying timber. Torrey.

Morellus. Fungus reticulate or cellular above, smooth underneath. 58. 1-esculentus, (morel) without volva, cellular outside, margin attached to stem.

Hezvelia. Fungus turbinate, plaited, wrinkled, inflated, deformed or concave, smooth, elastically ejecting seeds from the upper surface. 58. 1calyciformis, (smooth-cup fungus) ochre, glabrous, scurfy, stem short.

C.LAvaria. Fungus cup-form, erect, elongated, simple or branched: seeds dispersed over the whole surface, or collectcd in tubercles opening at top. 58. 1-cornea, (club fungus) red-orange, sim: ple or cloven.

Clathrus. Fungus roundish, cancellated with fleshy branches interwoven with each other. 58. 1-cancellatus, (latticed fungus) pilens open letticed.

Peziza. Fungus concave above; hemispherical or bell-form; seeds contained in the cup. 58. 1coccinea, (dish fungus) scarlet within, buff without.

Cyathus. Fungus bell-form or cylindric, bearing lentiform capsules within. 58. 1-striatus, (birinest fungus) dark brown, villous.

LYCOPERDON. Fungus becoming powdery and fibrous within: seeds attached intcrnally to the fibres. 58. 1-bovistum, (puff-ball) white becoming blackish, spherical. 
Furigo. Fungus with a cellular-fibrous bark; the fibres penetrating in a reticulate mannel through a fibrous mass. 58.1-fava, (soot fungus) yellow.

Stemonitis. Fungus covered with a fugaceous or membranaceous bark, filled with seminiferous fibres elastically expanding themselves. 58. 1-fascicularis, (brittle-bark puff-ball) steel-blue, head sub-turbinate, stem very short.

Aecrorum. Fungus furnished with membranaceous cases, glabrous on both sides, filled with naked seeds not cohering 58.1-cornutum, (cased fungus) yellow, cases long curved olive grey.

Spriaeria. Fungus furnished with cases filled with roundish naked gelatincus seeds. 58. 1-mo$r i$, (globule fungus) clustered scarlet small.

Sclerotium. Fungus quite simple, globularoblong, tough, bardish, gaping a little at length in the center, covered with an inseparable bark never opening above. 58. 1-semen, (barked fungus) subglobular, blackish, rugged.

UREDo. Fungus parasitical, consisting of a mealy powerer, destitute of a case, growing from under the cuticles of leaves and stems, bursting at last, with an even margin. 58. 1-frumenti, (blight, or grain-rust) linear oblong, black-brown, growing from under the cuticle of culms-fava, (yellow rust) sub-globular yellow, growing from under the cuticle of leaves.

Tubercularia. Fungus gelatinous : cup tubercled : stalk thick, filled, closely appressed, bearing the seeds on the upper stirface. 58. Ivulgaris, (tubercled fungus) convex rugged with a sub-repand-tooth margin.

Mucor, Fungus fugacious, heals at first semitrarsparent, then becoming opake, fixed to simple or branched stalks. 58. 1-mucedo, (common mould) globular, stem simple grey. 
Mosrla. Consisting of moniliform filaments clustered into a head. 58. 1-coesfittosa, (bead-fungus) branched with ternate spikes.

Xylostroma. Fungus leathery, expanded, deformed, penetrating, smooth, even : seeds among the fibres, globuiar minute. 58. 1-giganteum, (leather punk, or oak leather) fibres parallel, the swole fungus filling the interstices between the cleavages of decaying wood.

FINIS.

\section{INDEX OF THE GENERA.}

$\begin{array}{lrlr}\text { Acalypha } & \text { Page. } & \text { Alyssum } & 73 \\ \text { Acer } & 111 \text { Amaranthus } & 106 \\ \text { Achillaa } & 44 \text { Amaryllis } & 37 \\ \text { Acnida } & 95 \text { Ambrosia } & 105 \\ \text { Aconitum } & 115 \text { Ammania } & 19 \\ \text { Acorus } & 61 \text { Ammi } & 32 \\ \text { Acrostichum } & 40 \text { Amorpha } & 80 \\ \text { Actata } & 119 \text { Ampelopsis } & 28 \\ \text { Adiantum } & 58 \text { Amygdalus } & 54 \\ \text { Adonis } & 121 \text { Anagallis } & 22 \\ \text { Ecidium } & 64 \text { Anchusa } & 21 \\ \text { Eschynomene } & 141 \text { Andromeda } & 47 \\ \text { Esculus } & 85 \text { Andropogon } & 16 \\ \text { Agaricus } & 42 \text { Anemone } & 62 \\ \text { Agave } & 139 \text { Anethum } & 34 \\ \text { Agrimonia } & 38 \text { Angelica } & 32 \\ \text { Agrostemma } & 53 \text { Annona } & 63 \\ \text { Agrostis } & 51 \text { Anthemis } & 95 \\ \text { Aira } & 13 \text { Anthoceros } & 131 \\ \text { Aletris } & 14 \text { Anthoxanthum } & 9 \\ \text { Alisma } & 38 \text { Antirrhinum } & 71 \\ \text { Allium } & 42 \text { Apargia } & 86 \\ \text { Alnus } & 37 \text { Apium } & 34 \\ \text { Alopecurus } & 105 \text { Apocynum } & 29 \\ \text { Alsine } & 13 \text { Aquilegia } & 61 \\ \text { Althaea } & 35 \text { Arabis } & 73 \\ & 78 \text { Aracisis } & 80\end{array}$


Âralia

Arbutus

Arctium

A renaria

Arethusa

Argemone

Aristida

Aristolochia

Arnica

Aronia

Artemisia

Arum

Arundo

Asarum

Asclepias

Ascyrum

Asparagus

Aspidium

Asplenium

Aster

Astragalus

Athyrium

Atragene

Atriplex

Atropa

Atheropogon

Avena

Azalea

$B$.

35 Boehmeria 104

48 Boletus 140

89 Boltonia 94

49 Burago 21

98 Botrychium 124.

60 Brachystemum 65

13 Brassica 75

99 Briza $\mathbf{1 5}$

93 Bromus 16

55 Broussonetia. 114

21 Biyum 129

107 Buchnera $\quad 76$

16 Bunias 72

52 Buxbaumia 130

29 Buxus 105

58 C.

39 Cacalia 90

321 Cactus 54

120 Calla 107

93 Calicium 134

83 Callicarpa 17

122 Callitriche 7

64. Caltha 62

31 Calycanthus $\quad 58$

24. Campanula 26

15 Cannabis $\quad 115$

16 Capparis 60

25 Capsicum 20

Baemyces

Cardamine $\quad 75$

159 Cardiospermum 44

127 Carduus $\quad 88$

18 Carex 102

129 Carpinus $\quad 109$

69 Carthamus 88

13: Carum 34

91 Cassia 46

36 Castanea 169

So Catalpa 8

109 Caulophyllum 36

89 Ceanothus 27

70 Censtrus 27

119 Celtis 31

7 Cenchrus 12

$\mathrm{N} 2$ 
I N DEX.

Centaurea

Centaurella

Cephalanthus

Cerastium

Cercis

Cetraria

Chærophyllum

Chara

Cheilanthes

Cheiranthus

Chelidonium

Chelone

Chenopodiam

Chironia

Chrysanthemum

Chrysosplenium

Cichorium

Cicuta

Cimicifugz

Cinna

Sircaea

Cistus

Citrus

Clathrus

Clavaria

Claytonia

Clematis

Cleome

Clethra

Climacium

Clinopodium

Cnicus

Cochlearia

Coix

Collinsonia

Comarum

Cummelina

Comptonia

Conferva

Conium

Conostylus

Convallaria

Convolvulus

96 Conyza 90

18 Coreopsis 96

18 Coriandrum 31

52 Cornicularia 138

46 Cornus 19

137 Corydalis 79

33 Corylus 109

100 Cratægus 55

123 Crotolaria 80

75 Cucubalus 50

58 Cucumis 112

72 Cucurbita 112

30 Cunila 67

23 Cupressus $\quad 111$

91 Cuscuta 30

49 Cyathus 140

87 Cymbidium 93

33 Cynanchum 99

61 Cynara 87

7 Cynoglossum 21

9 Cyperus 11

59 Cypripedium 99

59 D.

140 Dactylis 14

140 Dalibarda 57

28 Datisca 117

62 Datura 23

36 Daucus 32

46 Deeumaria 53

128 Delphinium 61

68 Dentaria 74

88 Dianthus 49

73 Diapensia 24

102 Dicksonia 122

9 Dicranum 127

58 Didymodon 127

10 Diervilla 26

133 Digitalis 71

132 Digitaria 12

33 Dionaea 47

38 Dioscorea 116

39 Diospyros 117

23 Diphascum 127 
Dipsacus

Dirca

Dodecatheon

Dolichos

Doronicum

Draba

Dracaena

Dracocephalum

Drosera

E.

Echium

Elephantopus

Eleusine

Elymus

Endocarpon

Epigaéa

Epilobium

Epipactis

Equisetum

Erigeron

Erinus

Eriocaulon

Eriophorum

Erodium

Eryngium

Erysimum

Erythronium

Eupatorium

Euphorbia

Euphrasia

Euonyinus

Fagus

Fedia

Ferula

Festuca

Ficus

Floerkea

Fluvialis

Fontinalis

Fothergillia

Fragaria

Frasera
19 Fraxinus

114

132

14.1

79

130

92 Funaria

72

58 Galactia

67 Galanthus

83

35 Galega 83

Galium 19

22 Gaultheria 48

97 Gaura 43

14. Genista 80

14. Gentiana 30

134 Geranium 77

48 Gerardia 71

43 Geum 58

98 Glaux 29

125 Glechoma 67

92 Gleditschia 117

70 Glycine 83

104. Ginaphalium 90

11 Gordonia 77

77 Gossypium $\quad 78$

31 Gratiola 8

74. Grimmia 126

39 Gymnostomum $\quad 126$

89 Gyrophora 134

63 Hamamelis H. 20

27 Hamiltonia 115

Hedera 29

108 Hedysarum 84

10 Helenium 94

32 Helianthus 95

16 Heliotropium 21

114 Helleborus 62

37 Helonias $4 \mathrm{~L}$

100 Helvella 140

130 Hemerocallis 39

60 Hepatica 63

57 Heracleum 32

18 Hesperis 74 


\section{6}

Heuchera

Hibiscus

Hieracium

Hippophae

Hippuris

Holcus

Holosteum

Hordeum

Hortensia

Hottonia

Houstonia

Hudsonia

Humulus

Hyacinthus

Hydnum

Hydrangea

Hydrastis

Hydrocotyle

Hydropeltis

Hydrophyllum

Hyoscyamus

Hyoseris

Hypericum

Hypnum

Hypoxis

Hyssopus

Ilex

Impatiens

Imperatoria

Indigofera

Inula

Ipomaea

Iris

Isanthus

Isidium

Isnardià

Itea

Iva

Jasminum

Jeffersonia

Juglans
I N D X.

30 Juncus 10

78 Jungermannia $\quad 130$

86 Juniperus $\mathbf{1 1 8}$

114. Justicia 8

7 IS.

16 Kalmia 47

17 Krigia 87

13 Kuhnia 89

$\begin{array}{lll}50 & & \text { I. }\end{array}$

18 Lamium 65

52 Liathyrus 81

115 Laurus 45

39 Lavandula 65

140 Lechea 17

48 Lecidea 134

62 Ledum 48

31 Leersia 13

64. Lemna 100

22 Leontodon 86

24 Leonurus 66

87 Lepidium 73

60 Lepraria 132

129 Leptanthus $\quad 10$

37 Leskea 128

66 Lespedeza 84

Liatris 87

20 Ligusticum $\$ 2$

28 Ligustrum 8

33 Lilium 39

82 Limretis 14

92 Limodorum 97

25 Linnaea 18

10 Linum 55

64. Liquidambar 110

135 Liriodendron 63

19 Lihospermum 21

28 Lobelia 27

96 Lolium 15

Lonicera 26

8 Ludwigia 19

44 Lupintis 80

108 Lychis $\quad 52$ 
I N D E X. 147

Lycium

Lycoperdon

Lycopodium

Lycopus

Lygodium

Lysimachia

Lythrum

Magnolia

M.

Malaxis

Malva

Marchantia

Marrubium

Martynia

Maschalocarpus

Matricaria

Medeola

Medicago

Meesia

Melampyrum

Melanthium

Melilotus

Melissa

Melothria

Menispermum

Mentha

Menyanthes

Merulius

Mesembryanthemum

Mespilus

Mikania

- Milium

Mimosa

Mimulus

Mitabilis

Mitchella

Mitella

Mnium

Mollugo

Momordica

Monarda

Monila

Monotrapa

17 Morellus $\quad 140$

140 Morus 105

124. Mucor 141

9 Muhlenbergia 13

124 Myosotis 21

22 Myrica 114

52 Myriophyllum $\quad 106$

Myrtus 54

63 N.

98 Najas 100

78 Narcissus 37

151 Narthecium $\quad 40$

66 Neckera 1.8

72 Nelumbium 63

127 Neottia 98

94. Nepeta 66

41 Nicotiana 23

83 Nigeila 61

128 Nymphaea 60

69 Nysia 116

$41 \quad 0$.

84. Obolaria 69

67 Oenothera 4.3

113 Onoclea 119

117 Onopordon 88

66 Onosmodium 21

22 Opegrapha 133

139 Ophioglossim 124

56 Orchis 97

55 Origanum 68

90 Ornithogallum 40

13 Orobanche 69

77 Orontium 40

72 Orthotrichum 128

27 Oryza 40

18 Oryzopsis 12

49 Osmunda 123

129 Ostrya 109

17 Oxalis 51

112 Oxycoccus 43

9
142 Panax P. 30

48 Paeonia 60 
148

Panicum

Papaver

Parietaria

Parmelia

Parnassia

Passiflora

Pastiuaca

Pedicularis

Peltidea

Penthorum

Pentstemon

- Periploca

Peziza

Phacelia

Phalaris

Phascum

Phaseolus

Philadelphus

Phleum

Phlox

Phryma

Phyllanthus

Physalis

Phytolacca

Pinus

Pisum

Plantago

Platanus

Poa

Podalyria

Podophyllum

Podostemum

Polemonium

Polygala

Polygonum

Polymnia

Polypolium

Polvtrichum

Pontederia

Populus

Poitulacca

Potamogeton

Potentilla
I N D E, X.

12 Poterium 107

59 Pothos 19

104 Prenanthes 86

136 Primula 22

35 Prinos 36

76 Proserpinaca 17

34 Prunella 68

71 Prunus 54

137 Psilotum 124

51. Pteris - 120

7\% Pulmonaria 20

29 Pycnanthemum 65

140 Pyrethum 91

24. Pyrola 46

13 Pyrus 56

125 Pyxidanthera 25

82 Quercus $Q$, 107

14. Queria 17

24.2 R.

68 Ranunculus 63

111 Raphanus 75

25. Reseda 53

52 Rhamnus 27

110 Rheum 45

81 Rhexia 43

17 Rhinanthus 69

110 Rhododendron 47

15 Rhodora 46

4.5 Rhus 34

60. Rives 29

101 Riccia 131

24. Ricinus 111

79 Rivina 20

44 Robinia 82

96 Rosa 56

119 Rosmarinus 9

127. Rubia 18

38 Rubus 56

116 Ruribeckia 95

52 Ruellia 70

20 Rumex 4.2

57. Ruta 46 


\begin{tabular}{|c|c|c|c|c|}
\hline & & Sison & & \\
\hline Saccharum & 13 & Sisymbrium & & 76 \\
\hline Sagina & 20 & Sisyrinchium & & 76 \\
\hline $\begin{array}{l}\text { Sagittaria } \\
\text { Salicornia }\end{array}$ & 106 & Sium & & $\begin{array}{r}32 \\
116\end{array}$ \\
\hline $\begin{array}{l}\text { Salix ornia } \\
\text { Salix }\end{array}$ & 113 & $\begin{array}{l}\text { Smilax } \\
\text { Smyrnium }\end{array}$ & & 33 \\
\hline Saisola & 30 & Solanum & & \\
\hline Salvia & & Solidago & & 92 \\
\hline Sambucus & 34 & Sonchus & & \\
\hline Samolus & 26 & Sorbus & & \\
\hline Sanguinaria & 59 & Sorghum & & \\
\hline Sanguisorba & 18 & Sparganium & & \\
\hline $\begin{array}{l}\text { Sanicula } \\
\text { Sal }\end{array}$ & 31 & Sparganophorus & & \\
\hline Saponaria & 49 & Spartina & & 1 \\
\hline Sarothra & 35 & Spergula & & 5 \\
\hline Sarracenia & 59 & Sphaeria & & 14 \\
\hline Satureja & 66 & Sphaerophoron & & 13 \\
\hline Satyrium & 97 & Sphagnum & & \\
\hline Saururus & 43 & Spigelia & & \\
\hline Saxifraga & 48 & Spiloma & & 13 \\
\hline Scandix & 33 & Spinacia & & 11. \\
\hline Scheuchzeria & 41 & Spirdea & & \\
\hline Schizaea & 123 & Splachnum & & 12 \\
\hline Schoenus & 11 & Stachys & & \\
\hline Schwalbea & 70 & Staphylea & & \\
\hline Scirpus & $1 !$ & Statice & & \\
\hline Scleranthus & 49 & Stellaria. & & \\
\hline Scleria & 103 & Stemonitis & & 14 \\
\hline Sclerotium & 14.1 & Stereocaulon & & 159 \\
\hline Scolopendrium & 121 & Sticta & 12 & 137 \\
\hline Scrophularia & 70 & Stylosanthes & & \\
\hline Scutellaria & 67 & Swertia & & \\
\hline Secale & 14 & Symphitum & & \\
\hline Sedum & 51 & Syringa & & \\
\hline Selinum & 53 & $\mathrm{~T}$. & & \\
\hline Sempervivum & 54 & Tagetes & & \\
\hline & 93 & Trlinum & & \\
\hline & 104 & Tanacetum & & \\
\hline Sicyos & 112 & Taxis & & $1:$ \\
\hline Sida & 78 & Tetraphis & & 12 \\
\hline Silene & 50 & Teucrium & & \\
\hline Silphium & 96 & Thaelaephora & & 140 \\
\hline Sinanis & 76 & Thalictrum & & \\
\hline
\end{tabular}


150

Thelotrema

Thesium

Thlapsi

Thuya

Thymus

Tiarella

Tilia

Timmia

Tradescantia

Tragopogon

Trichodium

Trichostema

Trichostomum

Tricolhorum

Trientalis

Trifolium

Triglochin

Trillium

Triosteum

Tripsacum

Triticum

Trollius

Tropoelum

Tubercularia

Tulipa

Turritis

Tussilago

Typha

UImus

Ulva

Uniola

Urceolaria

Uredo

Urtica

Usnea
INDEX.

135 Utricularia s

29 Uvularia 39

$\begin{aligned} 73 & \text { Vaccinium } \\ 6 . & 47\end{aligned}$

68 Valeriana 10

49 Vallisneria 113

59 Variolaria 133

128 Veratrum 4.1

36 Verbascum 23

85 Verbena 65

14. Verbesina 95

67 Vernonia 88

127 Veronica 8

11. Verrucaria 134

42 Vexillatia 82

84. Viburnum 34:

42 Vicia 81

42 Vinca 25

27 Viola 28

101 Vitis 28

14. Vittaria 120

$64 . W$.

44 Woodsia 122

141 Wcodwardia 121

39 Xinthium X. 105

93 Xanthorhiza 36

101 Xylosteum 26

Xylostroma 142

Sl Xyris 11

132 Z.

136 Zapania 70

141 Zea 101

104, Zigarenus 4.1

138 Zizaniz 106 


\section{ENGLISH INDEX.}

$\begin{array}{lrlr} & \text { Page } & \text { Bear-berry } & \\ \text { Adder tongue } & 39 & \text { Beard-grass } & 13 \\ \text { A frican marigold } & 91 & \text { Beard-tongue } & 76 \\ \text { Agave } & 38 & \text { Beaver tree } & 63 \\ \text { Agrimony } & 53 & \text { Bed straw } & 19 \\ \text { Albany flax } & 104 & \text { Beech } & 108 \\ \text { Alder } & 105 & \text { Beech drops } & 48 \\ \text { Alexanders } & 33 & \text { Beet } & 31 \\ \text { Allum root } & 30 & \text { Bell-flower } & 26 \\ \text { Amaranth } & 106 & \text { Bell-wort } & 40 \\ \text { Althaea frutex } & 78 & \text { Bermuda-mulberry } & 17 \\ \text { American cowslip } & 62 & \text { Birch } & 109 \\ \text { American oil nut } & 115 & \text { black } & 109 \\ \text { American water-cress } & 76 & \text { canoe or paper } & 109 \\ \text { Angelica } & 32 & \text { white or broom } & 109 \\ \text { Apple } & 56 & \text { yellow } & 109 \\ \text { Arbor vitx } & 111 & \text { Birds nest } & 48 \\ \text { Arethusa } & 98 & \text { Birth-wort } & 99 \\ \text { Arnic } & 92 & \text { Bishop-weed } & 32 \\ \text { Arrow grass } & 42 & \text { Bitter nut } & 108 \\ \text { Arrow head } & 107 & \text { Bitter sweet } & 25 \\ \text { Artichoke } & 87 & \text { Bitter winter-green } & 46 \\ \text { Ash } & 114 & \text { Black-berry } & 57 \\ \text { Asparagus } & 39 & \text { Black flower } & 41 \\ \text { Atamask lily } & 37 & \text { Black-jack } & 108 \\ \text { Atragene } & 64 & \text { Black root } & 32 \\ \text { Avens } & 58 & \text { Bladder-campion } & 50 \\ \text { Balm } & 67 & \text { Bladder mouth } & 128 \\ \text { Balm of Gilead } & 117 & \text { Bladder-nut } & 34 \\ \text { Balsam apple } & 112 & \text { Bladder wort } & 8 \\ \text { Balsam fir } & 111 & \text { Blessed thistle } & 96 \\ \text { Balsam weed } & 28 & \text { Blight } & 141 \\ \text { Bane-berry } & 58 & \text { Blite } & 7 \\ \text { Barberry } & 36 & \text { Blood-root } & 60 \\ \text { Barley } & 13 & \text { Blue bottle } & 96 \\ \text { Bass-wood } & 59 & \text { Blue curls } & 67 \\ \text { Bay-berry } & 114 & \text { Blue-eyed grass } & 76 \\ \text { Bean } & 82 & \text { Blue gentian } & 67 \\ & \text { M } & \\ & & & \end{array}$


Blue grass

Bog rush

Bog sedge

Boneset

Borage

Boston iris

Bowman's root

Box

Box-flower

Box thorn

Brake

Brier-herb

Brittle bark puff-ball

Brook-weed

Broom corn

Broom grass

Broom-rape

Buchnera

Buck-bean

Buck-thorn

Buck-wheat

Bugbane

Bugloss

Bullrush

Burdock

Burnet

Burnet-saxifrage

Burr-marigold

Burr-reed

Bush clover

Bush trefoil

Butter-cup

Butterfly weed

Butternut

Button bush

Button wood

Caubage

Camphion

Canada mint

Canada thistle

Cancer root

Canterbury bells
15 Caper bush

11 Caraway

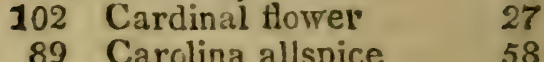

21 Carpet weed 17

10 Carrot 52

56 Cassia 46

105 Castor-oil plant 111

25 Catalpa tree $\quad 8$

18 Catchily 50

120 Catmint $\quad 66$

57 Cat-tail 101

141 Cedar 111, 118

26 Celandine 58

15 Celery .. 34

16 Centaury 24

69 Chaff seed $\quad 70$

71 Chamomile 95

22 Changeable hydrangea 50

27 Chara 100

45 Cherry

61 choak $\quad 54$

21 common 54

40 white 54

89 Chervil 33

107 Chestnut $\quad 109$

18 Chestnut oak $\quad 107$

89 Chess 16

101 Chick-weed 35

84. Chick-winter-green $\quad 42$

84 China aster 94

64. Chives 37

83 Choak berry $\quad 55$

108 Choak-dog $\quad 99$

18 Climbing-bonesel $\quad 90$

110 Clover 84

75 Club-moss $\quad 125$

52 Club rush 11

66 Cockfoot grass 12

88 Cock-roach mullein 23

69 Cockle 51

27 Colic weed $\quad 79$ 
Colt's foot

Columbine

Comb-tooth thistle

93 Decumary

61 Deer grass

88 Dill

Comfrey

Common mould

22 Dock

141 Dodder

95 Dog bane

Coriander

Corn spurry

Corol root

Cotton

Cotton grass

Cotton thistle

Cowbane

Cowhage

Cow-parsiey

Cowslip

Cow-wheat

Crab-tree

Cranberry

Cranbill

Creeping cucumber

Crow-foot

Crown-beard

Crown-cup

Cuckold

Cucumber

Cudweed

Currant

Custard apple

Cut grass

Cypress vine

Daffodil

Daisy

Dalibard

Dame's violet

Dandelion

Darnel grass

Date plum

Day flower

Day lily

31 Dog tail grass

51 Dog tooth violet $\quad 39$

98 Dog wood 19

79 Dragoness plant 38

11 Dragon head : 67

88 Duck meat $\quad 100$

33 Dwarf dandelion 87

32 Dwarf foxglove 71

32 Dyer's kneed grass 80

23 Dyer's weed 53

69 Dying sea weed 132

56 Eatable mushroom 139

43 Elder black berried 34

77 red berried 34

113 Elecampane 92

$63 \& 77$ Elephant foot 97

$95 \mathrm{Elm} 31$

89 Enchanter's nightshade 9

89. Endive 87

113 English water-cress 76

90 Erinus 70

29 Eschallots 37

63 Fvergreen snakeroot 79

14 Evergreen thorn 55

25 Eye bright 69

38 False acacia 82

91 False aloe 38

57 False asphodel $\quad 40$

74 False boneset 89

86 False chamomile 94

15 False cowslip 22

117 False foxglove 71

10 Faise gentian 30

38 False grape 28

Deadly night-shade 24 False gromwell 21

Dead nettle

65 False hawk weed 86 
False heath

ialse hemp

Tialse horehound

False indigo

False mermaid

False mustard

False saffiron

False sanicle

False sensitive plant

False sun-flower

Ialse syringa

Ealse toad-flax

Feather leaf

Tennel

Fennel flower

Fern

adder tongue

calyxed

caterpillar

fine haired

flowering

fork

fringed

grape

kidney fruit

lip

naked

one sided

yibbon

Roman

shield

snuff-box

Fescue grass

Fescue sedge

Feverfew

Feverwort

Field thyme

Fig tree

Fig wort

Finger grass

Five finger

Flat top

52 Flax 35

117 Fleabane 92

66 Floating arum 40

So Flower de luce $\quad 10$

37 Fog fruit 70

37 Follicle vine 29

83 Fothergill's bush 60

49 Four o'clock 27

85 Fox glove 71

24 Fox tail grass 13

54. French marigold 91

29 Frog's spittle 132

23 Fumitory 79

34 Fungus

61 barked 141

124

123

121

122

123

119

124

124

121

123

124

123

120

120

122

122

12

142

birdnest $\quad 140$

cased 14.1

club $\quad 140$

dish $\quad 140$

giobule 141

latticed 140

pink 140

smooth cup 140

soot 141

tubercled 141

Galingale

veined

139

Garlic

Gayfeather

11

37

88

Gentian 30

Giant fennel 32

102 Globe fower $\quad 34$

91 Goat's beard 86

27 Goat's rue 83

68 Golden rod 92

114 Golden saxifrage 49

70 Gold thread 62

$\approx$ Gourd 112

57 Grain rust 141

88 Grape vine $\quad 38$ 
I N D EX.

Grass

\begin{tabular}{|c|c|c|c|}
\hline & & sour & \\
\hline arrow & 42 & whitlow & \\
\hline beard & $13 \& 16$ & yellow eyed & \\
\hline lue & 15 & Grass pink & \\
\hline blue eyed & 76 & Greek valerian & \\
\hline oom & 16 & Green brier & \\
\hline kfoot & 12 & Green hair & \\
\hline tton & 11 & Gromwell & \\
\hline & 14 & Ground ivy & \\
\hline nel & 15 & Ground nut & \\
\hline & 44 & Ground pine & \\
\hline tail & 14 & Hacmatack & \\
\hline r's kneed & 80 & Hail grass & \\
\hline cue & 36 & Hairy beard grass & \\
\hline & 12 & Hard-hack & \\
\hline & 13 & Hazle nut & \\
\hline y beard & $14:$ & Heal all & \\
\hline gehog & 15 & seed & \\
\hline & $\begin{array}{l}12 \\
44\end{array}$ & $\begin{array}{l}\text { Hedgehog grass } \\
\text { Hedge hyssop }\end{array}$ & \\
\hline & 14 & Hedge mustard & \\
\hline dow & 15 & Hellebore swamp & \\
\hline ard & 14 & white & \\
\hline$=$ & 12 & Helonias & \\
\hline issus & 35 & Hemlock & \\
\hline per - & 73 & Hemlock tree & \\
\hline & 15 & Hem & \\
\hline & 16 & Henbane & \\
\hline & $\begin{array}{l}13 \\
40\end{array}$ & Herb bennet & \\
\hline & $\begin{array}{l}40 \\
12\end{array}$ & $\begin{array}{l}\text { Herb robert } \\
\text { Hibiscus }\end{array}$ & \\
\hline ion & 21 & Hickory & \\
\hline & 63 & High heal all & 7 \\
\hline ash & 15 & High water shrub & 9 \\
\hline & 102 & Hog weed & 106 \\
\hline & 16 & Holly & 2 \\
\hline & 37 & Holly hock & r \\
\hline vernal & 10 & Hone wort & 3 \\
\hline & 113 & Honey locust & $11 \%$ \\
\hline & 14 & ley suckle & \\
\hline & $\begin{array}{l}103 \\
N^{T}\end{array}$ & trumpet & 2 \\
\hline
\end{tabular}


Honey suckle

Hop

Hop horn beam

Hore hound

Horn beam

Horned popny

Horse balm

Horse chesnut

Horse radish

Horse tail

Hound tongue

House leek

Hyacinth

garden

Hydrangea grape

Hyssop

Ice plant

Indian corn

Indiin cucumber

Indian hemp

Indian mallows

Indian physic

Indian turnip

Indigo

Itch weed

Ivy

Jacob's ladder:

Jasmine

Jerusalem artichoke

Jewel weed

Job's tear

Jonquil

Judas' tree

Juniper

Knawell

Knot grass

Knot spurge

Labrador tea

Ladies' slipper

Ladies' traces

yellow

2
11
10
67
10
6

4
73
125
5
5

26

109

67

109

60 Lavander

9 Leaf cup

42 Leaf flower

73 Leaf seed

125 Leather leaf

21 Leather purik

54 Leather wood

39 Leek

39 Lemon

39 Leopard's bane

48 Less flowering rush

66 Lettuce garden

56

101

41

29

78

56

107

82

41

29

116

8

95

28

102

38

46

118

49

44

53

48

99

98

\section{tall}

white

Lichen button coral

crust

efflurescent $\quad 133$

filamentous 138

fungus 134

horned 138

Iceland 138

knobbed 139

leprous like $\mathbf{1 3 3}$

lettered 134

mitre 134

pit back $\quad 136$

pitcher shield 136

scarlet mouth 139

seed ball. $\quad 135$

shield $\quad 136$

small pox 133

spangled 134

target $\quad 136$

Lichnedia

24

90 


\begin{tabular}{|c|c|c|c|}
\hline Light haip & 11 & May weed & 95 \\
\hline Lilac & 8 & Meaciow grass & 15 \\
\hline Lily & 39 & Meaduw rue & 62 \\
\hline Lily orchis & 98 & Mediar & 55 \\
\hline Lime grass & 14 & Melilot clover & 84 \\
\hline Limodore & 97 & Mermaid weed & 17 \\
\hline Little snake wred & 104 & Mignonette & 53 \\
\hline Live forever & 51 & Milk parsley & 33 \\
\hline Liver leaf & 63 & Milk vetch & 83 \\
\hline Liver wort & 131 & Milk way plant & 83 \\
\hline brook & 131 & Milk weed & 99 \\
\hline gaping & 131 & Milk willow herb & 53 \\
\hline wax & 131 & Milk wort & 79 \\
\hline Lizard tail & 43 & Millet. & 13 \\
\hline Locust tree & 82 & Mithridate mustard & 73 \\
\hline Lombardy poplar & 117 & Mitre wort & 49 \\
\hline Loose strife & 22 & Mock orange & 54 \\
\hline Louse wort & 71 & Monkey flower & 72 \\
\hline Louse wort fox glove & 71 & Monk's hood & 61 \\
\hline Lovage & 32 & Moon seed & 117 \\
\hline Love lies bleeding & 106 & Morel & 140 \\
\hline Low calamint & 67 & Morning glory & 23 \\
\hline Iucerne clover & 83 & Moss & \\
\hline Lung wort & 20 & awl cup & 126 \\
\hline Lupine & 80 & bow & 129 \\
\hline Madder & 18 & creeping & 127 \\
\hline Mad wort & 73 & curve haired & 128 \\
\hline Magnolia & 63 & double tooth & 127 \\
\hline Maiden hair & 121 & earth & 126 \\
\hline Malabar nut & 8 & false & 131 \\
\hline Mallows & 78 & fork & 127 \\
\hline Maple & 44 & four toothed & 126 \\
\hline Maple leaf & 34 & goose neck & 129 \\
\hline Mare's tail & 7 & hair cap & 127 \\
\hline Marjoram & 68 & hair mouth & 127 \\
\hline Marsh five finger & 58 & leafless & 130 \\
\hline Marsh hibiscus & 78 & many speckled & 128 \\
\hline Marsh mallow & 78 & net tooth & 128 \\
\hline Marsh mallows & 78 & peat & 120 \\
\hline Marsh rusemary & 36 & pricked tooth & 129 \\
\hline Marsh tway blade & 98 & rope & 130 \\
\hline Master wort & 33 & sleeping & 120 \\
\hline
\end{tabular}


I $\mathrm{N} \mathrm{D} \mathrm{E} \mathrm{X.}$

Moss

spiral tooth
trait haired
thread
toothless
tree
umbrella
water

Mother of thyme

Mother wort

Mountain ash

Mountain mint

Mountain rice

Mouse ear

Mouse ear chickweed

Mulberry

Mullein

Mushroom

Musk melon

Mustard

Myrtle

Nasturtion

Neclace weed

Nelumba

Nettle

Nettle tree

New-Jersey tea

Nightshade

Nodding horehound

Oak

black
pin
red
rock or chesnut
scarlet
scrub
swamp
two coloured
white
yellow

Oak leaf foxglove Oak leather

\section{$I N D X$}

$\therefore$

Oak of Jerusalem

127 Oats 16

30

128 Okra 78

129 Onion 37

126 Orach 31

128 Orange 59

126 Orange root 62

130 Orchard grass 14

$\begin{array}{ll}68 & \text { Orchis } \\ 66 & \end{array}$

55 scarlet 97

$9 \& 65$ Orpine 51

12 Oswego tea 9

90 Ox-eyed daisy 91

55 Painted cup 69

105 Palma christi 111

23 Panic grass 12

139 Paper punk 140

113 Parnassus grass 85

76 Parsnip 34

54. Partridge berry 18

44. Passion flower 76

58 Pea 81

63 Peach 55

104. Pea nut 81

31 Pear 56

27 Pearl wort 20

25 Peat moss 126

67 Pea vine 83

107 Pellitory 105

108 Pencil flower 85

108 Penny royal 67

108 Penny wort 69

107 Peony 60

108 Pepperidge $\quad 116$

108 Pepper grass 73

107 Pepper mint 66

108 Periwinkle 25

108 Persicaria 45

108 Pheasant's eye 64

71 Phryma 68 
Pickerel weed

Pig weed

Pine

pitch

white

yellow

Pine weed

Fink

Pink root

$P$ in weed

Pipe wort

Plane tree

Plantain

Plantain sedge

Plowman's wort

Plum

Poison hemlock

Poison ivy

Poison sumach

Poke weed

Polypod

Pond lily

Pond weed

Popla․

balsam

high

lombardy

white

Poppoose root

Poppy

Potatoe

Prickly ash

Prickly pear

Prim

Primrose

Puff ball

Pumpkin

Purslane

Pyramid flowel

Quake grass

Quick set

Quince
38 Rabbit foot $\quad 84$

30 Radish 75

110 Ragged cup $\quad 96$

110 Rag wort 93

110 Rape 73

110 Raspberry ... 57

35 Rattle box 80

49 Red clover 84

23 Red pepper 26

17 Red top 13

104 Reed grass 16

110 Reed mace 101

17 Rhodora $\$ 6$

103 Rhubarb 45

90 Ribbon grass 13

54 Rib wort 17

33 Rice 41

35 River nymph $\quad 100$

34. Rocket 74.

52 Rock rose - $\quad 59$

119 Rose 56

60 Rose bay 47

20 Rose cockle $\quad 51$

117 Rosemary 9

117 Round head 104

117 Rue 46

117 Rue anemone 62

117 Ruel 70

56 Rush grass 40

59 Rye 14

25 Sage. 9

115 Salt grass : 12

54. Salt wort 30

8 Samphire 7

23 Sand wort 50

140 Sanicle $\quad 32$

112 Sassafras 45

52 Satyrion $\quad 97$

18 Savin : 118

15 Saxifrage 48

55 Scabish 43

56 Scarlet pimpernel . 23 
I N D X

Scorpion grass

Scouring rush

Screw stem

Scurvy grass

Sea buckthorn

Sea burdock

Sea holly

Sea milk-work

Sea pea

Sea rocket

Sea rush grass

Sea weed

Sedge

Seed box

Self heal

Seneka snake root

Sensitive plant

Sensitive polypod

Service tree

Sesame grass

Shad flower

Sheep berry

Shepherd's purse

Shin leaf

Side saddle flower

Silk weed

Silver cinquefoil

Single seed cucumber

Skull cap

Skunk cabbage

Slippery elm

Snake head

Snap dragon

Snow ball

Snow drop

Soap wort

Soft grass

Solomon's seal

Sorrel

Sour gum

Southern wood

Sow thistle
21 Spear mint

125 Speedwell

18 Spice bush

73 Spicy winter green 48

114 Spider wort 36

105 Spikenard

31 Spinach

29 Spindle tree

120

73 Spotted cranebill 77

15 Spruce 111

132 single 111

102 double 111

19 Spurge 53

68

79

77

119

55

102

55

34. St. Andrew's cross

73 Star flower

46

59

99

58

112

67

19

31

72

70

34

37

49 Succulent chick weed 17

16 Sugar cane 13

39 Sugar maple 44.

42 Sumach 34

116 Summer corn 101

91 Summer savory 66

87. Sundew
115

9

8

5

\section{8}

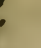

1

53

53

53

112

112

112

27

58

93

37

40

50

56

61

76

51

21

77

58

57

\section{7}

\section{3}

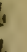


Sunflower

Swamp rose

Sweet brier

Sweet cicely

Sweet fern

Sweet flag

Sweet gale

Sweet gum

Sweet pea

Sweet pepper bush

Sweet potatoe

Sweet vernal grass

Sweet william

Swine lettuce

Syrian mallows

Taliny

Tall b'ue bottle

Tansy

Tape grass

Tare

Teasel

Thin grass

Thistle

Thorn apple

Thornbush

Thorough wor:

Threadfoot

Three seed mercury

Thyme

Tick seed sunflower

Timothy grass

Toad flax

Tobacco.

Tooth ache tree

Tooth cup

Tooth root

Touch me not

Touch wood

Tower mustard

Trailing arbutus

Tree moss

Tree primrose
95 True sarsaparilla 116

56 Trumpet flower 70

56 Tulip 39

33 Tulip tree 63

103 Tupelo tree 116

40 Turnip 75

114 Turnsole 21

110 Twin leaf 44

81 Unicorn plant 72

46 Valerian 10

23 Veiny hawk weed 87

10 Venus' fly trap 47

49 Venus' pride 18

37 Vervain 65

.78 Vetch 31

53 Violet 28

96 Virginian loosestrife 43

91 Virginian orpine 51

113 Virginian thyme 65

81 Virgin's bower 62

19 Wake robin 42

14. Walking leaf 121

88 Wall cress 74

23 Walnut black 108

55 shagbark 108

89 Walnut leaved ash 114

101 Water arum $\quad 107$

111 Water crowfoot 64

68 Water hemp $\mathbf{1 1 5}$

96 Water horehound 9

15 Water leaf 22

71 Water melon 112

23 Water milfoil 106

115 Water navel wort 31

19 Water nymph $\quad 100$

74. Water oats 106

28 Water parsnip 32

140 Water pepper 45

74. Water plantain 42

48 Water purslane 20

138 Water radish 76

43 Water shield 63 
Water star wort

Weeping willow

Wheat

summer

winter

Whip grass

White ash

White bush

White cedar

White clover

White lettuce

White radish

White snake root

White wood

Whitlow grass

Whortleberry

Wila basil

Wild bush bean

Wild caraway

Wild chamomile

Wild feverfew

Wild flag

Wild germander

Wild ginger

Wild honeysuckle

Wild indigo

Wild iris

Wild liquorice

Wild mandrake

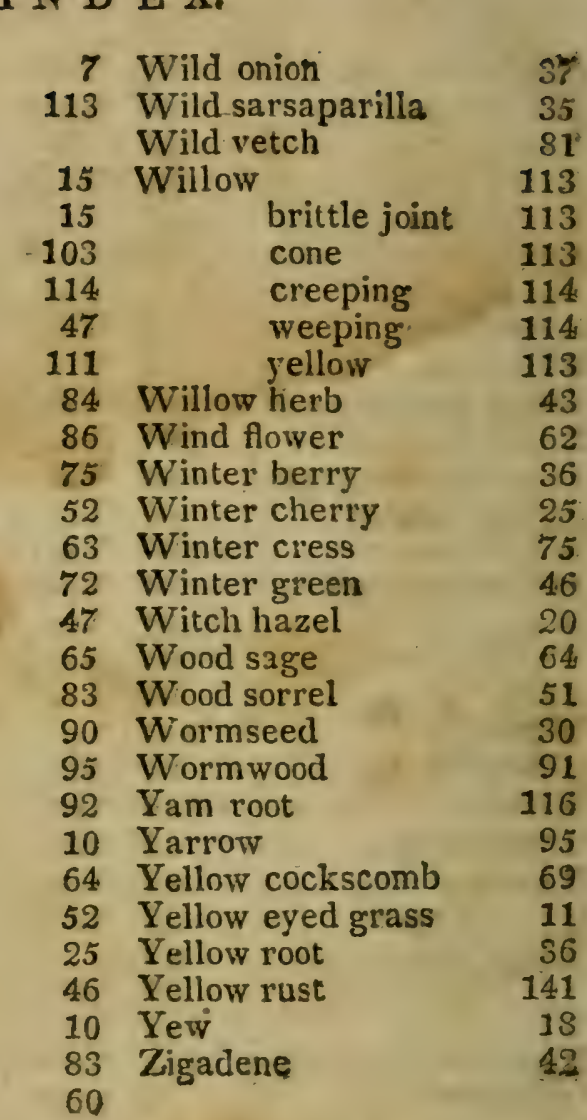

60 


\section{ERR.1T.}

Tне Address. For "Mason Fussell," read Frissell.

The Preface. For "Turton and Linneus," read Turton's Linneus.

On the 5th page, for "generic nouns," read generic names.

Correct the following by the heln of the Index:

AGAVE. For "virginca," read virginica.

Alisma. Over this genus erase "POLYANDRIa", and insert Polygynia.

Axchusa. For $h$ read $2 \psi$.

ANDROPOGON. For "stainate," read staminate.

Atriplex. Erase "(stem woody)" and insert (stems woody) over the genus Ulmus, as in page 26.

Browus secalinus. Read calyx azunless, \&c.

CARUMr. In the asterisked genera following this genus, set a period after Scleranthus.

Celastrus For "climbing," read truining.

Citrus, Var. limon. For "(lemon)" read (lime.)

Clavaria. For " cup-form," read club-form.

Conves alba. For $2 !$ read $h$. Over this gea nus insert, D. Flowers 4-petalled, sufzerior.

Dactruis. This genus must be considered as following Sorghum on the next page.

Dodecantheon. Read Dodecatheon.

Elymus. Strike out the words "4-leaved, 2flowered" and "ternate."

Gratiola. For Calyx 7-leaved, the 2 outer ones spreading :" read, Calyx 5-leaved, sometimes. with a 2-lcazed calycle. 
Inula. For "halenium," read helenium.

LEPTANTHUS. Erase" 3.1. " at the end of the generic description.

Lonicera dioica. For $\neq$ read $h$.

Magnolia: For 4 read $h$.

Marrubrium. Read Marrubium.

Prxidanthera... For $h$ read 2 .

Rubus. For "juices," read juicy.

SMyrium. In both species for $h$ read $\psi$.

SpIraEA. For "(hand-hack)" read (hard-hack.)

Thlaspr. For "roundish-cordate," read roundish-obcordate.

Trifolrun. Read 1 to 4-secded. And for "rapens" read repens.

Veroxica. For h read $\psi$.

Zapaxia. In the section placed over this genus, for "Leaves," read Calyxes.

The reader will not be surprised at this list of crrors, when he is told, that the author resides 34 miles from the printer, and never saw any of the proof sheets.

The alterations in the characters of the genera, Elymus and Gratiola, were caused by a perusal of the first numbers of that incomparable work, Elliot's Botany of South Carolina and Georgia. 


lay

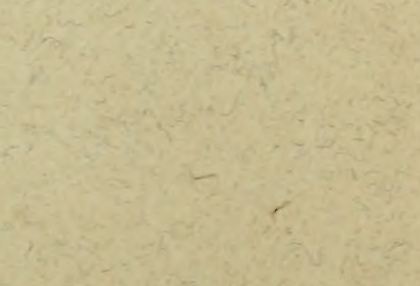



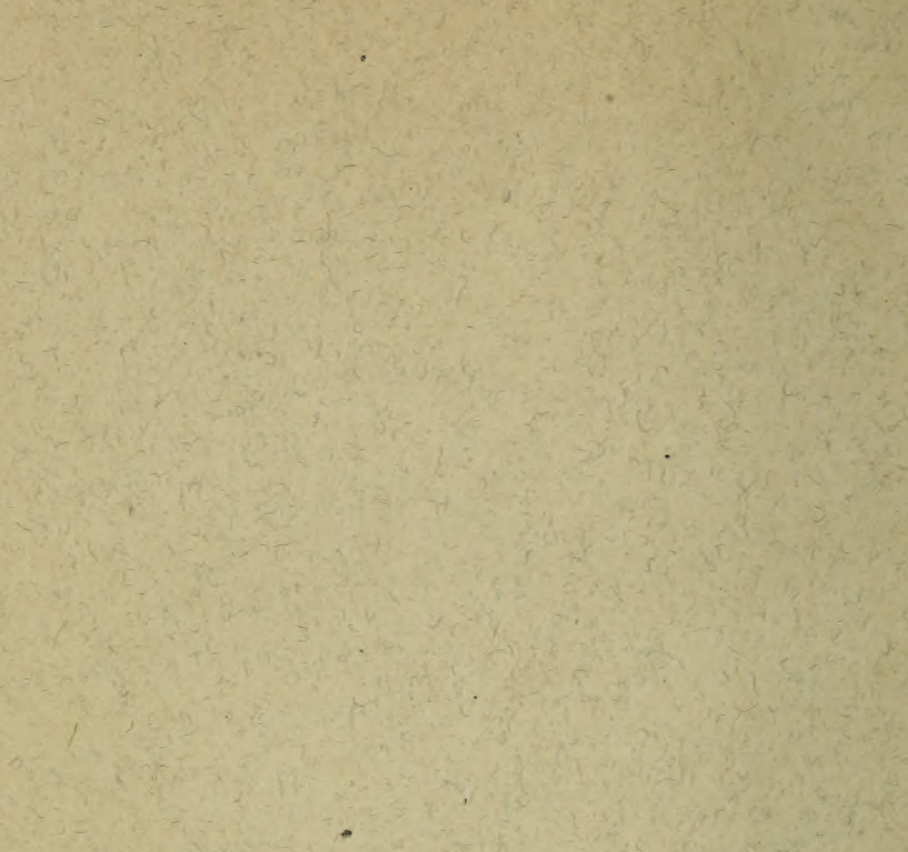

$3 y^{2}=$

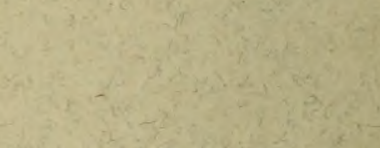




\section{QK110.E18}

QK110.E18 18 Botanical Garden Library

aton, Amos/A manual of botany for the $n$

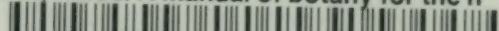

35185000873511 


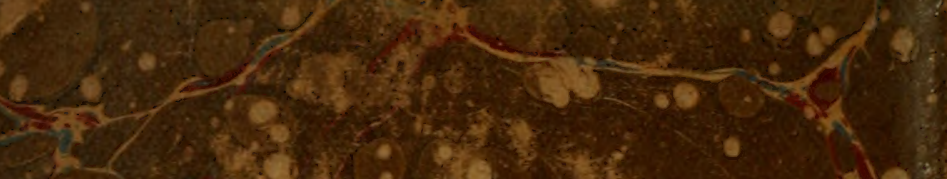

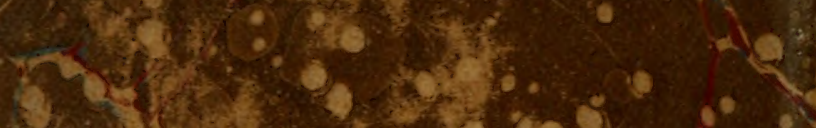

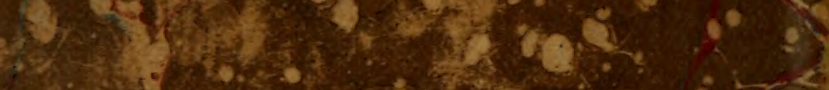
Q6.

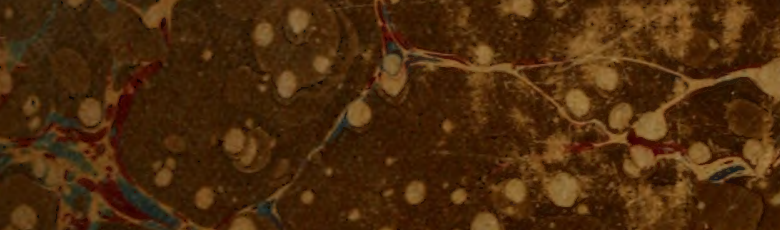

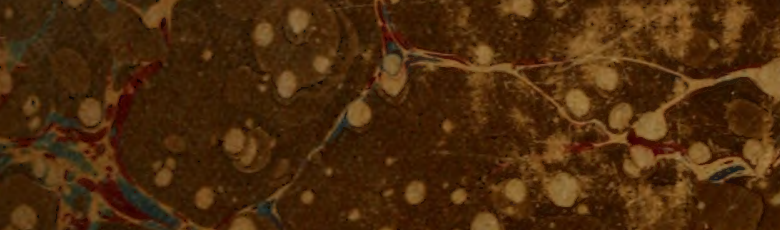

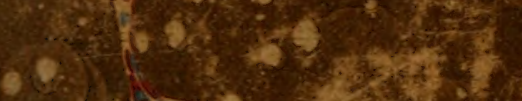

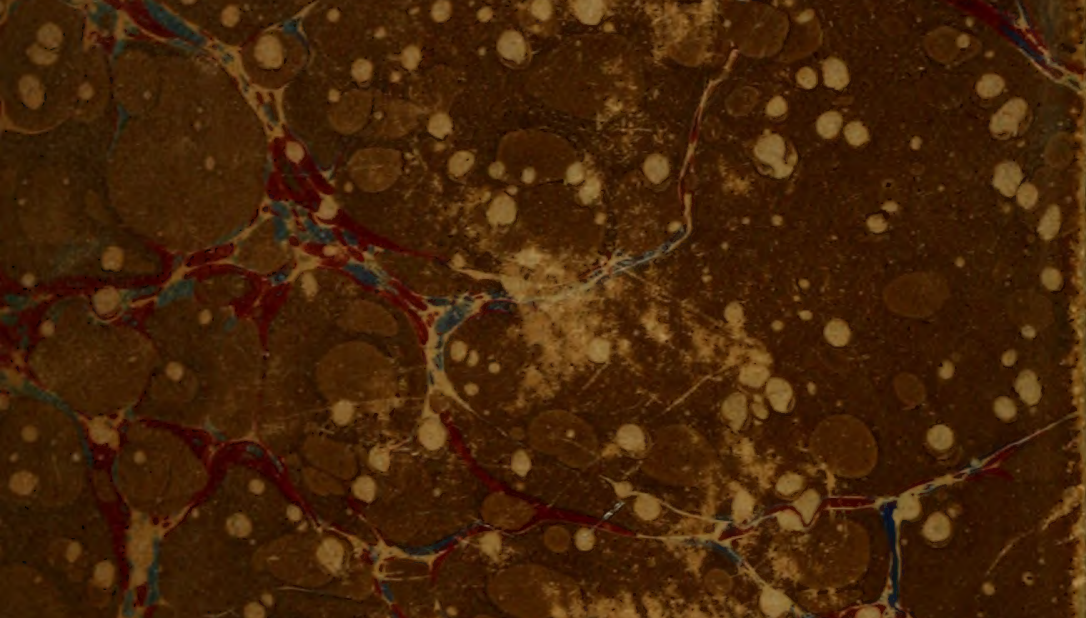

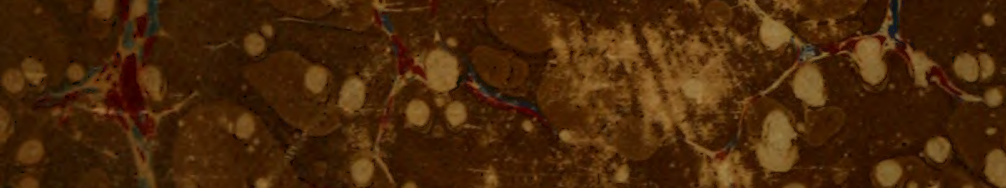

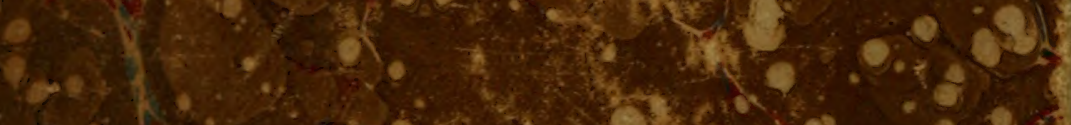

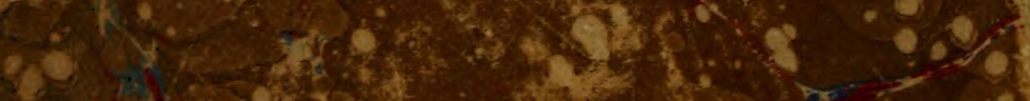

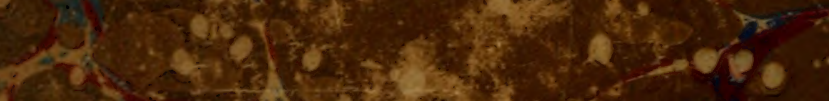

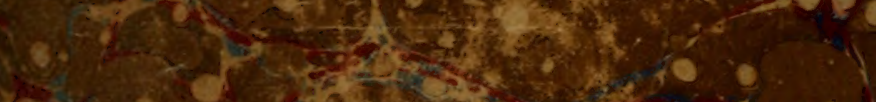

$\lim ^{2}$

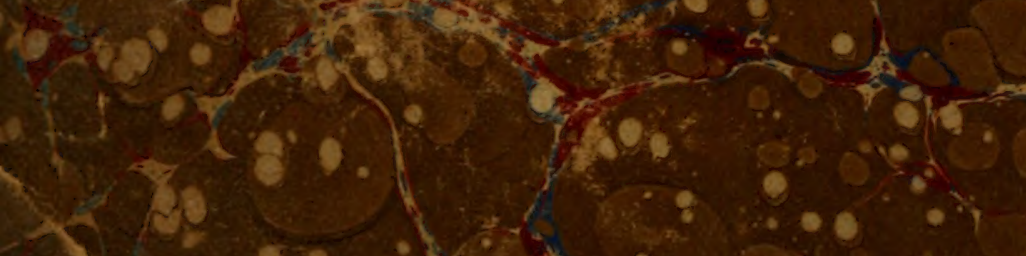

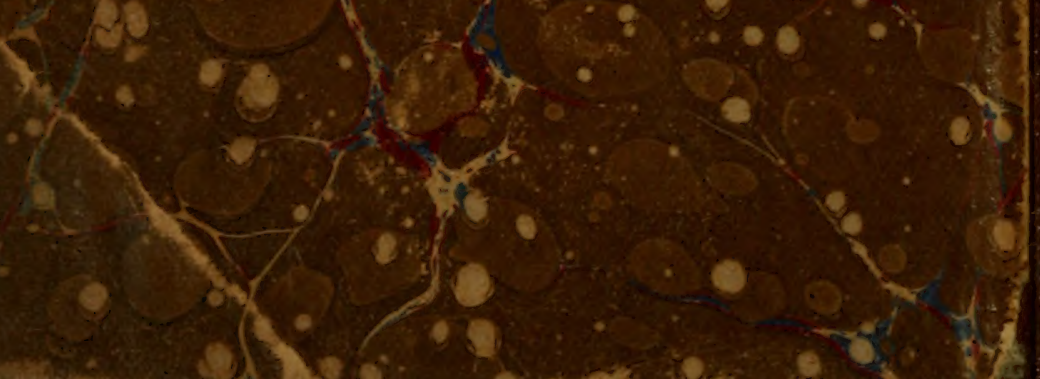

